Rochester Institute of Technology

RIT Scholar Works

Theses

$12-2014$

\title{
Optical simulation of terahertz antenna using finite difference time domain method
}

Chao Zhang

Follow this and additional works at: https://scholarworks.rit.edu/theses

\section{Recommended Citation}

Zhang, Chao, "Optical simulation of terahertz antenna using finite difference time domain method" (2014). Thesis. Rochester Institute of Technology. Accessed from 


\title{
Optical simulation of terahertz antenna using finite difference time domain method
}

\author{
by
}

Chao Zhang

A Thesis Submitted in Partial Fulfillment of the Requirements for the Degree of Master of Science in the Chester F. Carlson Center for Imaging Science

College of Science

Rochester Institute of Technology

December, 2014

Signature of the Author

Accepted by

Director, M.S. Degree Program

Date 


\section{CHESTER F. CARLSON CENTER FOR IMAGING SCIENCE \\ COLLEGE OF SCIENCE \\ ROCHESTER INSITITUTE OF TECHNOLOGY \\ ROCHESTER, NEW YORK}

CERTIFICATE OF APPROVAL

\section{M.S. DEGREE THESIS}

The M.S. degree Thesis of Chao Zhang

has been examined and approved by the

thesis committee as satisfactory for the

thesis requirement for the

Master of Science degree

Dr. Zoran Ninkov, Thesis Advisor

Dr. Robert Kremens, Committee Member

Dr. Alan Raisanen, Committee Member

Date 


\section{ACKNOWLEDGEMENTS}

At first, it is my great pleasure to work with my thesis advisor, Professor Zoran Ninkov, for his valuable advice and guidance through my two-year experience at CIS. He is always encouraging and often tells me to start work from the most fundamental physics and then to more deep thinking. His enthusiasm and passion to the research have a great influence on me. Not a single word could express my gratitude to him.

Thank Dr Robert Kremens and Dr Alan Raisanen for kindly be the committee members and taking time to review this thesis.

As members of the research project, Paul Lee, Andy Sacco, Dan Newman, Kenny Fourspring and others always inspire me to make progress. Greg Fertig, Dmitry Vorobiev, Ross Robinson often offer help in the lab. Wish all of them happy life and successful career.

Thanks to the faculty, staff and students at CIS for I had a great time with all of them. As an international student, they compose most of my life here.

Finally, my parents are the persons from whom I could obtain courage and comfort. Wish them to be healthy and expect them to enjoy the life. 


\begin{abstract}
Terahertz science is a promising and rapidly developing research area. However, solid state terahertz detectors of high performance are still needed. An antenna within each pixel is needed in these detectors so as to couple more incident radiation into the detector. In this thesis, a software package called Lumerical FDTD Solutions is used to optimize the terahertz antenna design. The ultimate goal is to design broadband antennas that work efficiently over desired frequency bands.
\end{abstract}

The transmission/absorption characteristics of various bowtie antennas were modeled using the software. For absorption modeling, an equivalent resistor was added to load the antenna and absorb the terahertz energy. The effect of various parameters, including geometrical shape, boundary condition, material index, were considered. Fat bowtie was chosen as the optimum design for a $215 \mathrm{GHz}$ antenna. Optimization was carried out to check how the gap, slot, distance between metal contacts would affect the performance of the antenna. A transmission experiment was designed to verify the validity of these simulations using a $188 \mathrm{GHz}$ source. Finally, some tests for the angular response of silicon/air interface and dipole antenna were done, in order to ascertain the efficiency of coupling between the optical telescope used to collect the $\mathrm{THz}$ radiation and the antenna/detector combination. 


\section{TABLE OF CONTENTS}

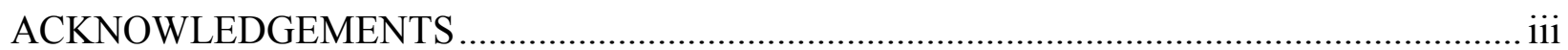

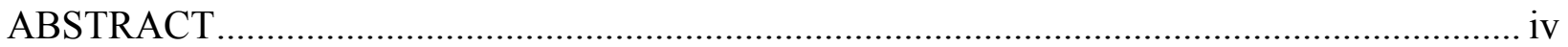

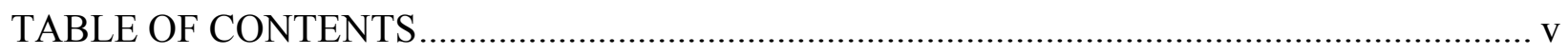

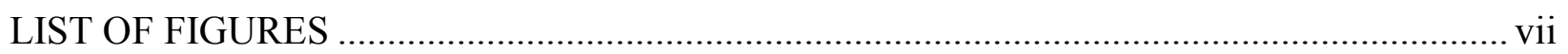

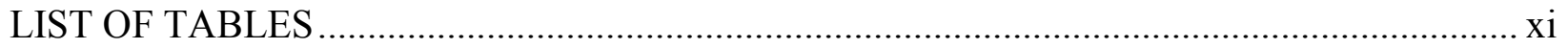

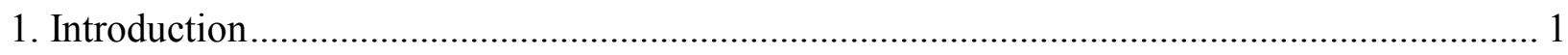

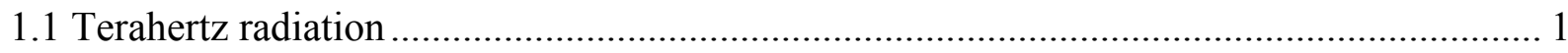

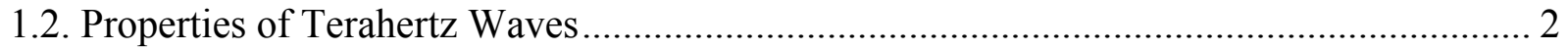

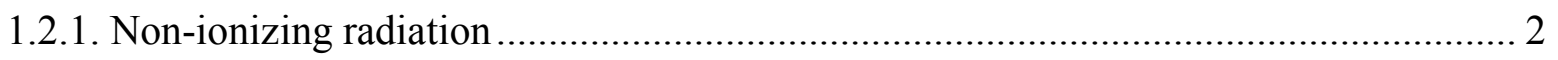

1.2.2. Terahertz penetration and transmission ......................................................... 3

1.2.3. Some other terahertz wave properties .................................................................. 4

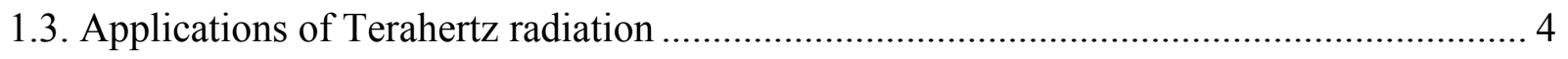

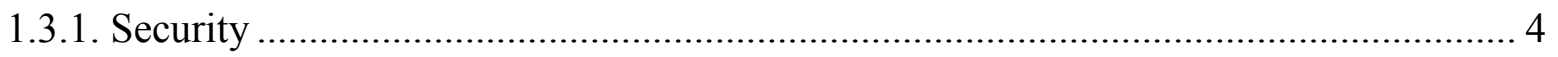

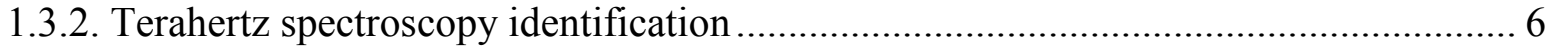

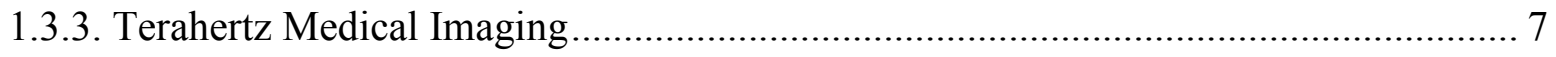

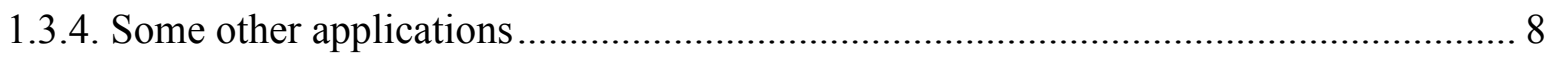

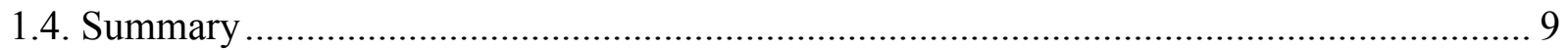

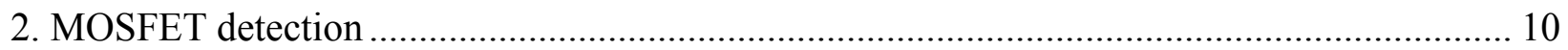

2.1. Direct and heterodyne THz detection ................................................................ 10

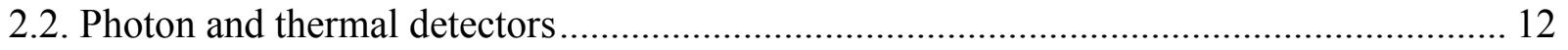

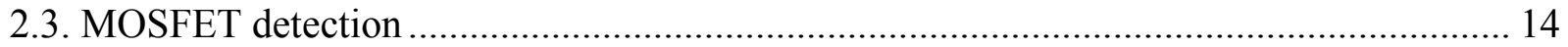

3. Numerical method, realization and simulation model ................................................... 18

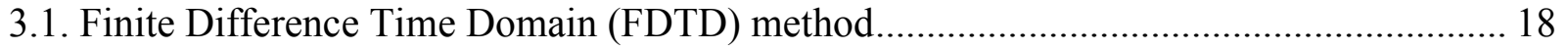

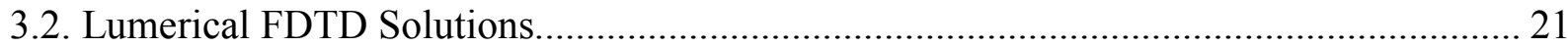

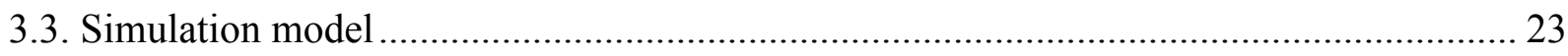

4. Effects of different parameters on the antenna performance ............................................... 29 


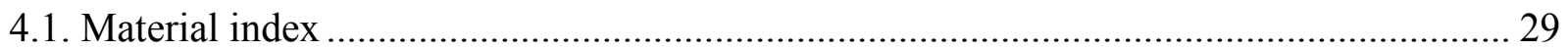

4.2. Comparison of simulation results to existing references ................................................. 34

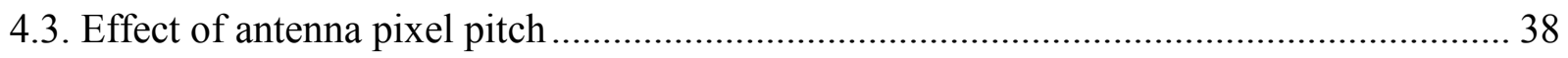

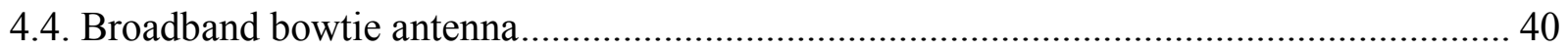

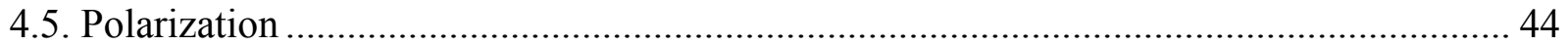

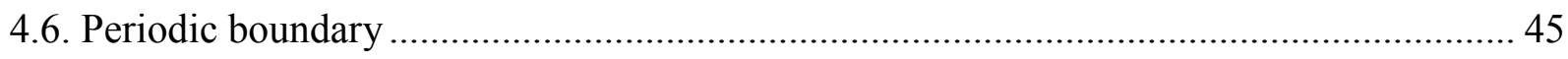

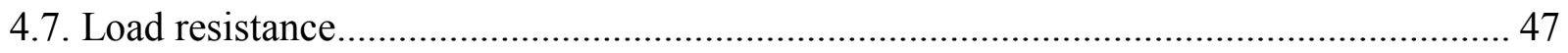

4.8. E-field distribution from antenna to detector ................................................................... 48

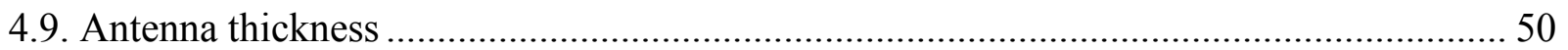

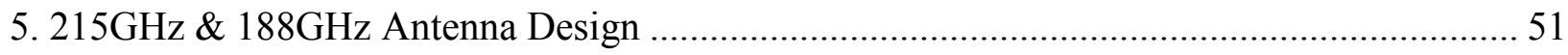

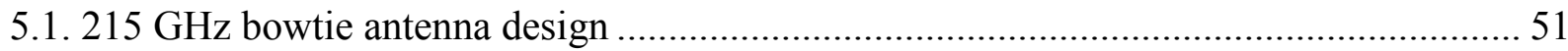

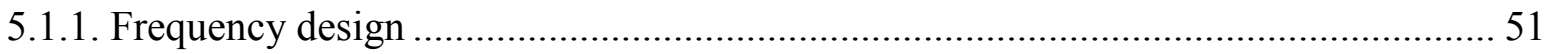

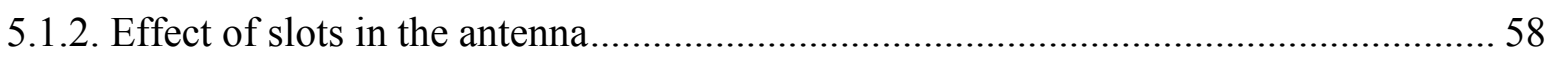

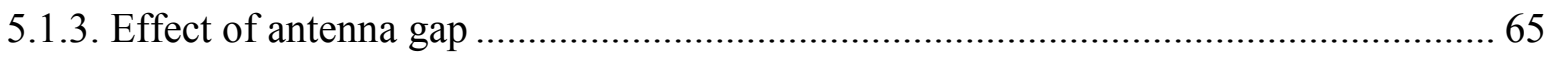

5.2. $188 \mathrm{GHz}$ transmission experiment design ................................................................. 71

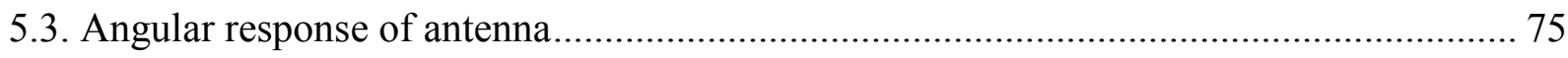

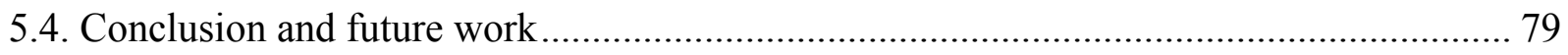

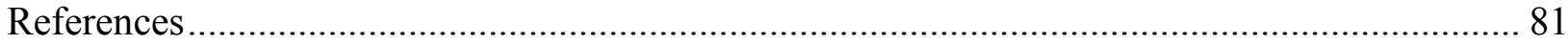




\section{LIST OF FIGURES}

Figure 1.1. Terahertz region in the electromagnetic spectrum [2]

Figure 1.2. Non-ionizing property of Terahertz wave

Figure 1.3. Absorption spectra of different explosives (left) and other substances (right) measured at THz frequencies [9]

Figure 1.4. Measured and simulated transmission spectra for a THz path length of $0.45 \mathrm{~m}$ at an ambient temperature of 20 degree centigrade and $30 \%$ humidity [11]

Figure 1.5. ThruVision TS5 people screening system (upper) and Weapon detection of a person with a hammer [13]

Figure 1.6. A tiny terahertz detection device detecting the hidden bullets inside a toy bear [14]

Figure 1.7. Mini-Z THz Time Domain Spectrometer [15]

Figure 1.8. THz spectroscopy identification of substance sealed in a letter. Screenshots from the introduction video of Mini-Z [15]

Figure 1.9. Imaging results of a tissue for $\mathrm{THz}$ (left) and Visible (right) [16]

Figure 1.10. THz image of an artificially contaminated chocolate bar: a stone (solid circle), a M2 metal screw (dashed circle) and a glass splinter (dotted circle) [19]

Figure 2.1. Schematic representation of a direct detection. $\mathrm{W}_{\mathrm{S}}$ is the signal power and $\mathrm{W}_{\mathrm{B}}$ is the background radiation power. [20]

Figure 2.2. Schematic representation of heterodyne detection

Figure 2.3. Schematic diagram of thermal detector

Figure 2.4. Schematic diagram of bolometer

Figure 2.5. n-MOSFET diagram [29]

Figure 2.6. Metal-oxide-semiconductor structure on p-type silicon

Figure 2.7. Plasma oscillations in MOSFET [20]

Figure 2.8. MOSFET THz detection with biased voltage, however, the biased current between source and drain is not included

Figure 3.1. Illustration of a standard Cartesian Yee cell in FDTD method (left) and Maxwell's equations which will be discretized (right).

Figure 3.2. 1D FDTD calculation principle

Figure 3.3. The GUI of FDTD Solutions

Figure 3.4. Top view of a bowtie antenna (left) and a spiral antenna (right) we try to model (not to scale)

Figure 3.5. Profile of transmission simulation scheme

Figure 3.6. Profile and 3D pattern of absorption simulation scheme (not to scale)

Figure 3.7. Distribution of 9 different antenna designs of Tiny Imager 4. Each type of antenna has 3-by-3 units and the size of each unit is 100-by-100 square microns 
Figure 3.8. Top view of six spiral antennas 1-6 (From top to bottom, left to right, 1 and 2 are actually of the same pattern. Not to scale.)

Figure 3.9. Side profile of detailed structure of Tiny Imager 4. The two arms of the antenna are connected to the terminals of MOSFET by several metallic and via layers. The material of via layer is tungsten. The index data in the diagram may not be the actual values.

Figure 4.1. Time domain spectroscopy (TDS) measurements of crystalline high-resistivity silicon from [44]. (a)

Power absorption coefficient, (b) index of refraction

Figure 4.2 . Multi-coefficient fitting for the oxide index data from 0.5 to $3 \mathrm{THz}$

Figure 4.3. Real part of aluminum index information comparison between the experimental data and the Al-Drude model from reference [46]. Imaginary case also agrees well but was not given.

Figure 4.4. Transmission, Reflection and their sum for Bowtie antenna 1 (left) and Bowtie antenna 3 (right) using PEC model

Figure 4.5. Comparisons of transmission (left) and reflection (right) results between the three models for Bowtie antenna 1 (top) and Bowtie antenna 3 (bottom)

Figure 4.6. Microscopic images of fabricated devices of the spiral type with different winding numbers (From the reference paper [50])

Figure 4.7. Transmission results of spiral antenna with winding number 3 (the second one of Figure 4.6) from the reference (left, blue line is for simulation and red line is for experiment) and from our simulation (right)

Figure 4.8. Figure 2 from [51], field intensity spectra in the gap of antennas

Figure 4.9. Transmission curve of cross antenna for vertical polarization from [50]

Figure 4.10. Transmission (red) and absorption (black) curves of three bowtie antennas (top left for Bowtie antenna 1, top right for Bowtie antenna 2, bottom left for Bowtie antenna 3) with unit pixel to be 100-by-100 square microns

Figure 4.11. Transmission curves of Bowtie antenna 1 with different periods from 100 to 500 microns (horizontal and vertical periods are the same)

Figure 4.12. Absorption curves for two bowtie antennas (both with a 50-ohm resistor) of different periods. The unit of period is micron. The inner and outer widths of antennas are 1 and 3.1 microns. They have a 2-micron gap, the length of one arm are 18.6 and 33.2 microns respectively.

Figure 4.13. Sketch of the Bowtie antenna mentioned in the report of the French group [53]

Figure 4.14. Schematic of Fat Bowtie antenna modeled here

Figure 4.15. Absorption curves for fat bowtie antennas restricted within a circle of which diameter is 150 microns

Figure 4.16. Absorption curves for fat bowtie antennas restricted within a circle of which diameter is 200 microns

Figure 4.17. Absorption curves for fat bowtie antennas restricted within a circle of which diameter is 250 microns

Figure 4.18. Linear equation fitting between the antenna diameter and resonant wavelength. $\mathrm{x}$ is diameter of the fat bowtie antennas and $\mathrm{y}$ is the corresponding resonant wavelengths.

Figure 4.19. Pixel photograph of French group design [53] 
Figure 4.20. Four different incident polarizations

Figure 4.21. Absorption curves of the fat bowtie antenna for different polarizations

Figure 4.22. Transmission curves of Bowtie antenna 1 for two cases (reducing period versus adding more antennas for periodic boundary case) and their difference

Figure 4.23. Absorption curves of Bowtie antenna 1 for two cases (reducing period versus adding more antennas

for periodic boundary case) and their difference

Figure 4.24. Absorption results for simulations checking the effect of periodic boundary. Three simulations have $1 * 1$,

$2 * 2,3 * 3$ antennas/pixels within the boundary respectively.

Figure 4.25. Absorption curves for Bowtie antenna 3 (except that the length is changed to be 40 microns for one element) for different resistances

Figure 4.26. Side profile of antenna with the locations of the three monitors indicated. Three monitors (M1, M2,

M3) are put across the metal contact to detect the E-field distribution information. Not to scale.

Figure 4.27. M1 E-field distribution. Unit of E field is $\mathrm{V} / \mathrm{m}$.

Figure 4.28. M2 E-field distribution. Unit of $\mathrm{E}$ field is $\mathrm{V} / \mathrm{m}$.

Figure 4.29. M3 E-field distribution. Unit of $\mathrm{E}$ field is $\mathrm{V} / \mathrm{m}$.

Figure 4.30. Absorption curves for Bowtie antenna 1 (pixel pitch is $100 * 100$ square microns) of different aluminum thicknesses from 0.5 to 1.5 microns

Figure 5.1. Linear equation fitting between the antenna length and resonant wavelength for first generation bowtie antennas

Figure 5.2. Schematic of one arm and central resistor for the 6 antenna designs. Not to scale.

Figure 5.3. Absorption responses for antennas of different lengths

Figure 5.4. Absorption responses for antennas of different angles

Figure 5.5. Absorption responses for antennas of different gaps

Figure 5.6. Absorption response comparison between two different oxide models

Figure 5.7. Absorption response comparison considering oxide and nitride configuration

Figure 5.8. New definitions for the antenna gap and angle (not to scale)

Figure 5.9. Absorption for a new defined bowtie antenna with length $=110$ microns, angle $=30$ degrees and gap $=10$ microns

Figure 5.10. Test structures to check the slot effect. Here, only one arm of the antenna is shown, where the etched slots are of several rectangles inside the arm. The widths of the etched slots are 2, 5 and 10 microns respectively.

Figure 5.11. Absorption simulations for antennas with test slots

Figure 5.12. Two tests for antennas with etched blocks. The sizes of the blocks are $5 * 5,30 * 40$ square microns respectively.

Figure 5.13. The antenna design with open-end slots and its absorption response. The width of the slots is 2 microns

Figure 5.14. The designs that satisfy the manufacture rule and after corners are rounded 
Figure 5.15. Comparison between solid antenna with sharp corners and slot antenna with rounded corners

Figure 5.16. Electric field distribution @ $215 \mathrm{GHz}$ for the central plane of the slot antenna with rounded corners (the antenna is along the horizontal direction). Electrical field unit: $\mathrm{V} / \mathrm{m}$

Figure 5.17. 1-D electric field distribution. Electrical field unit: V/m

Figure 5.18. Field distribution for antennas with gap $=5$ microns and inner width from 5 to 40 microns. Electrical field unit: $\mathrm{V} / \mathrm{m}$

Figure 5.19. Electric field distribution from reference [56]

Figure 5.20. Absorption responses when the gap is changed from 10 to 20 microns

Figure 5.21. Electric field distribution of the central plane through the antenna for variations of the gap between the two arms. Electrical field unit: V/m

Figure 5.22. Two different configurations for the final antenna design with a gap of 2 microns (not to scale)

Figure 5.23. Response comparison between two resistor configurations

Figure 5.24. E-field distribution (@215THz) in a highlighted area (yellow dash line box) of Configuration 2. Electrical field unit: $\mathrm{V} / \mathrm{m}$

Figure 5.25. Current density distribution (@215THz) in the highlighted area in Figure 5.24. Current density unit: $\mathrm{A} / \mathrm{m}^{2}$

Figure 5.26. Two sets of antenna designs for $188 \mathrm{GHz}$ transmission experiment. In the first set, there is a gap between two arms of the antenna. Certain implant could be added to define an equivalent resistor. In the second set, there is no equivalent resistor and the two arms are directly connected with each other.

Figure 5.27. Transmission curves for two sets of antennas (left, the green line represents location of $188 \mathrm{GHz}$ ) and the transmission values at $188 \mathrm{GHz}$ (right)

Figure 5.28. Transmission and reflection of air/silicon interface for radiation of different incident angles

Figure 5.29. Comparison between theoretic and simulation results for transmission and reflection of air/silicon interface as a function of incident angle. Blue curves are of theory and red stars are of simulation results. Source frequency is $0.18 \mathrm{THz}$. For data points of angle up to 60 degrees, the number of PML layers is 48 ; for all other cases with angle larger than 60 degrees, the number of PML layers is set to be 96 .

Figure 5.30. Left: Angular absorption response of a dipole antenna. The antenna includes a resistor to absorb energy and the absorption is equal to one minus reflection and transmission ( $A=1-\mathrm{R}-\mathrm{T})$. Right: An example of E field radiation pattern of a half-wave dipole antenna [59]. The antenna elements are located along the $270^{\circ}$ and $90^{\circ}$ Line.

Figure 5.31. Comparison between theoretic and simulation results for absorption of dipole antenna as a function of incident angle. Blue curve is of theory and green stars are of simulation results. Source frequency is $0.18 \mathrm{THz}$. Different from Figure 5.29 and 5.30, here 90-degree corresponds to the normal incidence and small angles represent larger incident angles. For data points of angle up to 25 degrees, the used number of PML layers is 48; for all other cases with angle larger than 25 degrees, the number is set to be 24 . 


\section{LIST OF TABLES}

Table 4.1. Resonant frequency/wavelength information for fat bowties of three different diameters

Table 5.1. Resonant frequency information for the three bowtie antennas of the first generation. The first row is length for one arm of the antenna. Since the gap between the two arms is 2 microns, the antenna length (the second row) is two times the values in the first row and then plus the gap.

Table 5.2. Geometric parameters of the 6 antenna designs for the modeling

Table 5.3. Antenna parameters for $188 \mathrm{GHz}$ transmission experiment. Period indicates the size of one pixel pitch. Array represents the number of antennas in $\mathrm{x}$ and $\mathrm{y}$ directions (the array size of each type of antenna is about $1.5 * 1.5$ square centimeters). 


\section{Introduction}

\subsection{Terahertz radiation}

Terahertz radiation $(\mathrm{THz})$, or T-ray, generally refers to electromagnetic waves with frequencies between 0.1 to $10 \mathrm{THz}$ (wavelength: 30 to 3000 microns, energy: 0.4 to 40 $\mathrm{meV}$ ) [1]. Terahertz waves locate itself between the traditional research regimes of electronics and photonics in the electromagnetic spectrum, microwave and infrared to be more specifically. The relative location could be seen in Figure 1.1. Since terahertz waves are in the wavelength range from 0.03 to 3 millimeter, terahertz radiation is sometimes knows as the submillimeter waves, especially in astronomy.

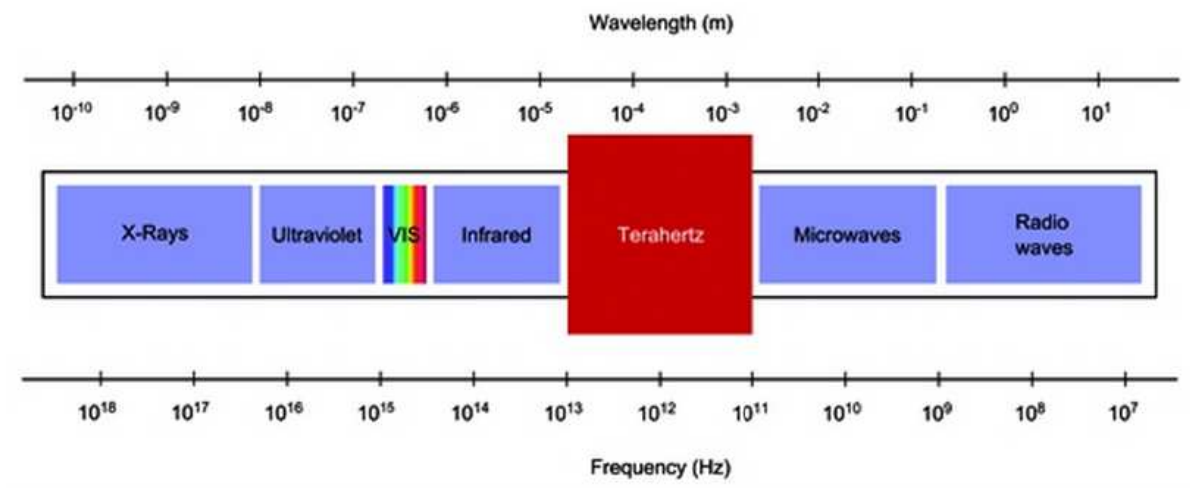

Figure 1.1. Terahertz region in the electromagnetic spectrum [2]

Compared to the neighboring regions in electromagnetic spectrum, the technology for generating and manipulating terahertz wave is in its infancy. Well established classical infrared technology is inapplicable since the wavelength seems too large from the optical perspective. Also, similarly, the expertise at lower frequencies is useless because the wavelength is small for that case. Hence, new devices and techniques need to be developed for the 'Terahertz Gap'. Fortunately, the last decades have observed the most intense research work into this regime [3-7]. 
The motive to explore this less developed area is rather because of some uniquely attractive features of terahertz waves than just simply closing the 'Terahertz Gap'. The properties of terahertz waves will be seen in Section 1.2.

\subsection{Properties of Terahertz Waves}

Terahertz waves have some remarkable properties which could lead to valuable applications in various fields. This section elaborates on these properties to offer perspective for the subsequent terahertz applications.

\subsubsection{Non-ionizing radiation}

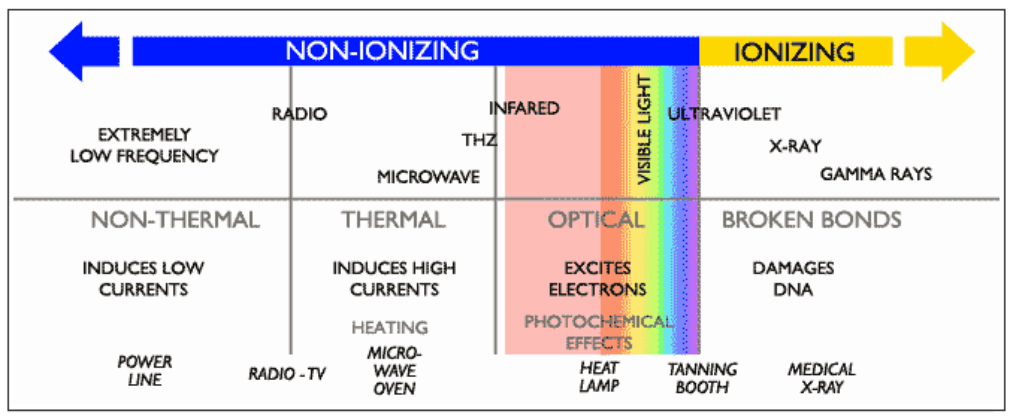

Figure 1.2. Non-ionizing property of Terahertz wave

Terahertz waves are non-ionizing radiations, meaning that they do not have enough energy to free electrons from an atom or molecule during interaction with a tissue or material. It is intuitive that waves with larger frequency have more energy per quanta and therefore could be ionizing and destructive. A well-known example of ionizing radiation is $\mathrm{x}$-radiation. It is a concern that large does or frequent exposure to $\mathrm{x}$-ray may result in mutation, cancer or even death. Observing Figure 1.2, $\mathrm{THz}$ radiation has longer wavelength and hence belongs to a safer region of the spectrum. This feature makes Terahertz a potential alternative for the diagnosis and treatment to replace $x$-ray screening. 


\subsubsection{Terahertz penetration and transmission}

Another primary advantage of terahertz wave is that many common materials and living tissues are transparent or semi-transparent to light in this range [8]. Terahertz radiation can penetrate through some non-conducting materials, like cloth, paper, wood, plastic and ceramics, which may otherwise be opaque for radiation at other frequencies. Moreover, many subjects feature characteristic absorption lines, therefore making it possible to do identification by observing the Terahertz fingerprints.
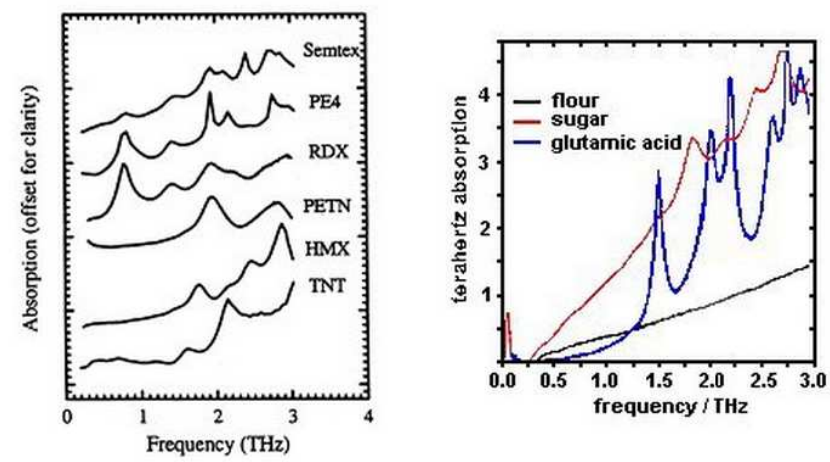

Figure 1.3. Absorption spectra of different explosives (left) and other substances (right) measured at $\mathrm{THz}$ frequencies [9]

An important obstacle for terahertz detection is that terahertz waves have limited penetration through fog and cloud and can not penetrate liquid water or metal [10]. Figure 1.4 shows a measurement example for the atmospheric transmission. For space-based detector, this effect would go away and would not be a concern since air is scarce there. Otherwise, it should be carefully considered.

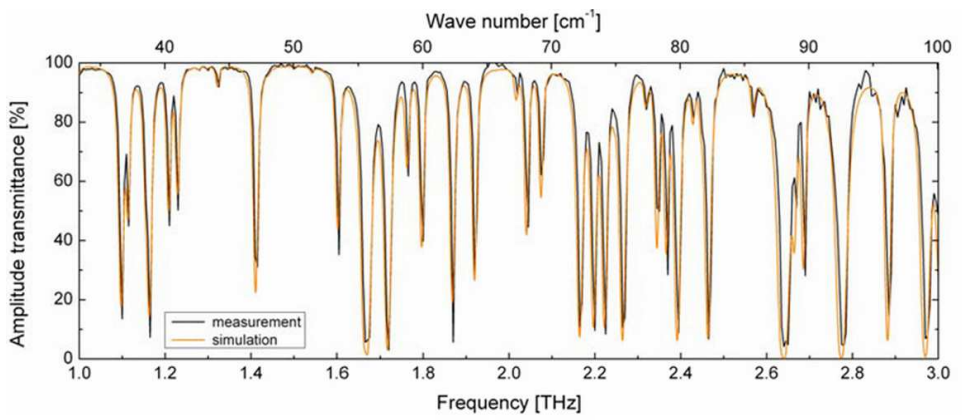

Figure 1.4. Measured and simulated transmission spectra for a THz path length of $0.45 \mathrm{~m}$ at an ambient temperature of 20 degree centigrade and $30 \%$ humidity [11] 


\subsubsection{Some other terahertz wave properties}

For one imaging system, according to the Rayleigh criterion, the minimum resolvable angle of a circular aperture is proportional to the wavelength and is inversely proportional to the diameter of the aperture. Therefore, compared to the current popular millimeter wave scanner device, one system utilizing the terahertz wave could have much better resolution and could be manufactured to be very compact. On the other hand, although the traditional optical imaging system, like x-ray device, has finer resolution, penetration characteristics as well as photon energy should also be taken into account. Subwavelength resolution could also be achieved [12].

Also, considering that Rayleigh scattering intensity is inversely proportional to the fourth power of the wavelength, $\mathrm{THz}$ wave scatters less than that of the optical radiation (optical section has shorter wavelength). This is favorable to get an image with better image quality.

\subsection{Applications of Terahertz radiation}

After reviewing the properties of the terahertz range radiation in the last section, we will examine some practical applications.

\subsubsection{Security}

As mentioned in the penetration characteristics of terahertz wave, this radiation could pass through fabric, plastic, cloth, etc. This feature makes it ideal for surveillance applications, for example, safety check at airports or government buildings. Terahertz imaging system is able to 'see' forbidden and hidden objects remotely. This is of particular interest since the travel flow keeps increasing and $\mathrm{THz}$ waves are able to produce images with better spatial resolution. Also, considering that terahertz radiation has a high chemical sensitivity or many 
chemical/biological subjects have specific terahertz fingerprints, we can combine spectroscopic techniques to detect the composite of certain item concealed in a container. This is a very promising application to replace the existing mm-wave and $\mathrm{x}$-ray scanner systems in that it provides better penetration, better resolution, no harm to human tissue and do not require contact to the examined subject.

ThruVision TS5, the product of a company called Digital Barriers, is a stand-off people screening unit able to do real-time concealed threat detection. It provides effective detection of larger objects at standoff distances up to $15 \mathrm{~m}$ and smaller items at distances up to $10 \mathrm{~m}$ [13]. Figure 1.5 shows a picture of the TS5 system and one sample image.
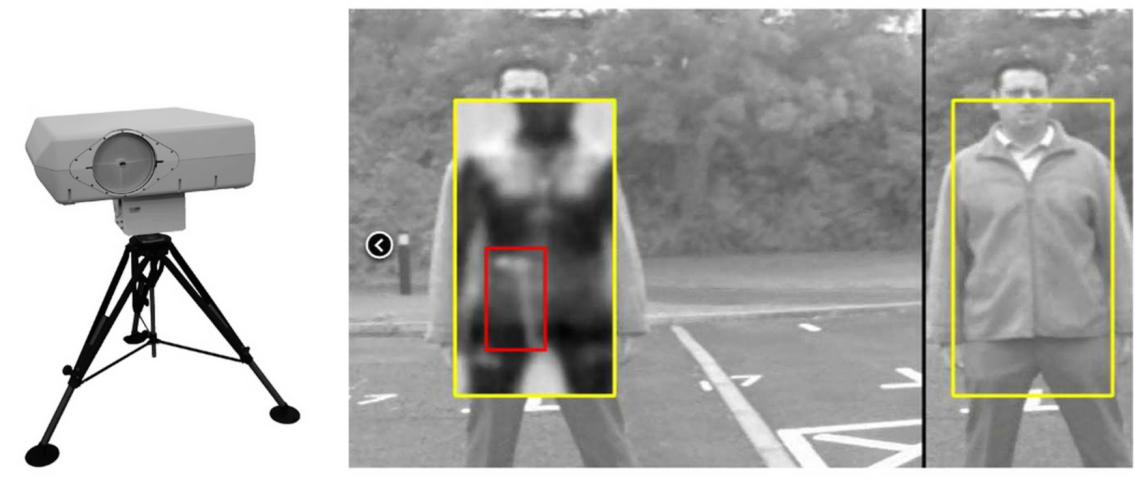

Figure 1.5. ThruVision TS5 people screening system and Weapon detection of a person with a hammer [13]
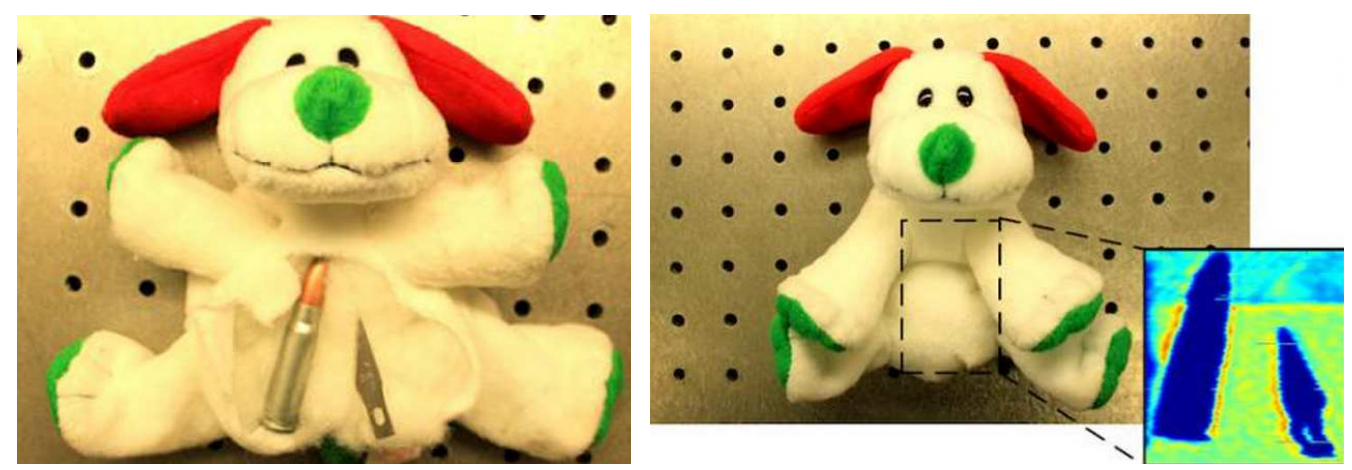

Figure 1.6. A tiny terahertz detection device detecting the hidden bullets inside a toy bear [14] 
Another example is a low cost, imager chip that could see through a toy bear for identifying bullets inside it [14]. This is shown in Figure 1.6.

\subsubsection{Terahertz spectroscopy identification}

Spectroscopic analysis of terahertz radiation is able to provide novel information in chemistry and biochemistry. Many materials have absorption lines in the terahertz range, facilitating the identification or differentiation by the spectral responses. This is also useful for mail/package screening since people become more worried there may be some dangerous substance that may threaten receiver's health.

In Figure 1.7, we see a compact $\mathrm{THz}$ time domain spectrometer, made by Zomega Company [15].

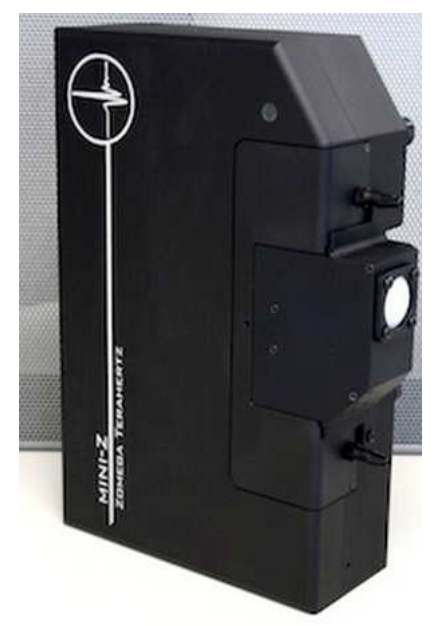

Figure 1.7. Mini-Z THz Time Domain Spectrometer [15]

This product is good for spectroscopic and non-destructive inspection applications in transmission or reflection. The experiment illustrated in Figure 1.8 shows how to identify whether the material sealed in a mail letter is safe or not. An absorption spectrum is measured from 0.1 to $1.5 \mathrm{THz}$. Then the absorption curve is identified and a decision is made on what the sample is. 


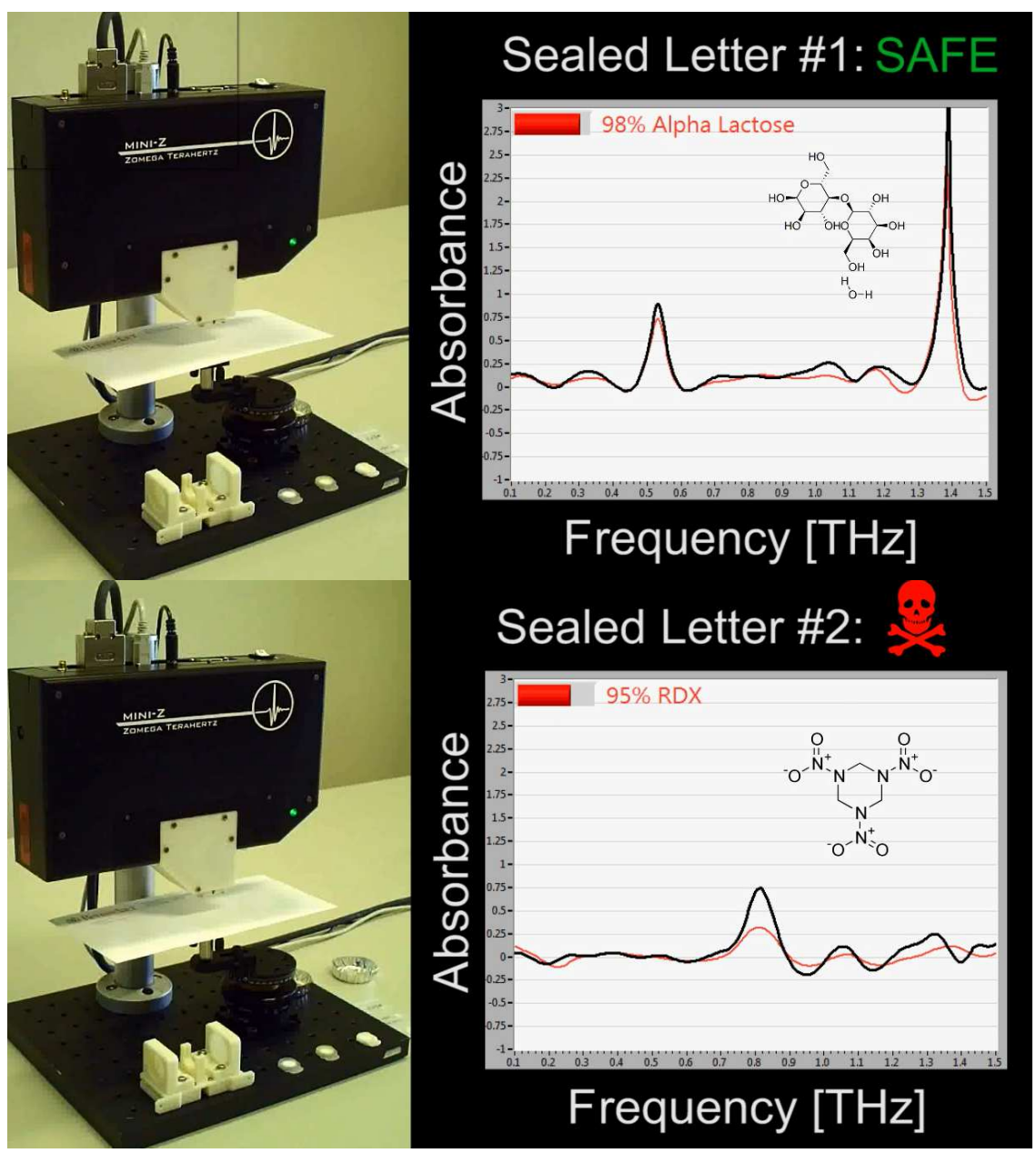

Figure 1.8. THz spectroscopy identification of substance sealed in a letter.

Screenshots from the introduction video of Mini-Z [15]

\subsubsection{Terahertz Medical Imaging}

Terahertz waves have some good properties to be applied for medical imaging. As a safe, non-ionizing and sensitive detection regime, this technique has some advantages over x-ray and CT scanning in some applications and may even replace them finally. Considering terahertz's ability to recognize spectral fingerprints, it could provide good contrast between different types of soft tissues. Terahertz imaging is also a sensitive method of detecting water content and other markers of cancer. Due to strong water absorption, terahertz waves only penetrate a small distance and allow high-resolution subsurface imaging of tissue. 
Terahertz Pulsed Imaging technique of the TeraView system could produce 3D images of tissues at high resolution [16]. It uses molecular markers, like water, combining spectral and absorption information to differentiate between healthy and unhealthy tissues. This would make more accurate boundaries and more importantly may have an earlier diagnosis than other methodologies.

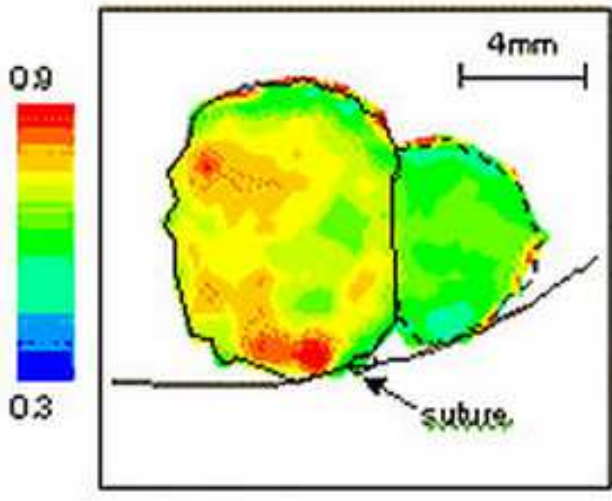

THz image

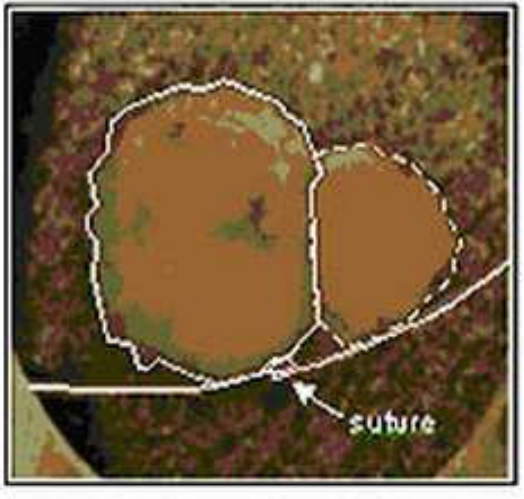

Visible Image

Figure 1.9. Imaging results of a tissue for THz (left) and Visible (right) [16]

The difference between these two images is that it is much easier for the observer to distinguish cancerous and non-cancerous cells in the first one while the second one seems more uniform. This application is definitely meaningful for that early detection would greatly increase the likelihood of cure.

\subsubsection{Some other applications}

Terahertz radiation also has applications in wireless communication systems [1718]. However, it is applicable at higher altitude for that the water vapor at lower altitude would greatly absorb the signal in this frequency range. Also, the usefulness in long-distance communication is restricted. 
In the manufacturing industry, $\mathrm{THz}$ systems could perform the quality control and the process monitoring job. This is based on the fact that most packaging materials are transparent to terahertz waves. Therefore, terahertz is able to detect the defects residing in the transparent materials which would not be detectable for sources of some other frequencies. In Figure 1.10, three defects could be easily detected by the THz image [19].

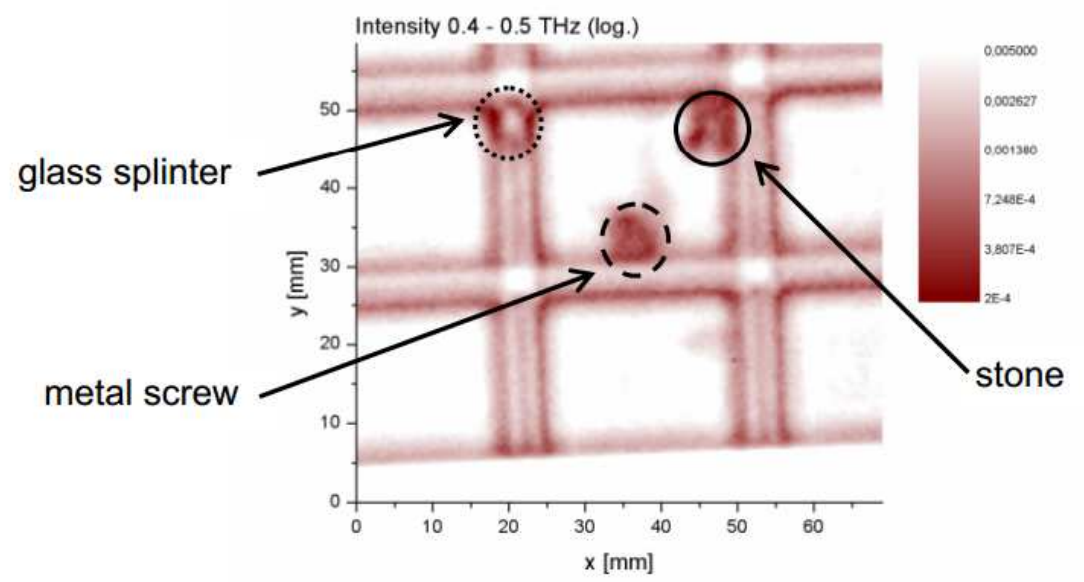

Figure 1.10. THz image of an artificially contaminated chocolate bar: a stone (solid circle), a M2 metal screw (dashed circle) and a glass splinter (dotted circle) [19]

\subsection{Summary}

Terahertz range radiation, as a relatively undeveloped interval in the electromagnetic spectrum, is posing various exciting applications. Non-ionizing, penetration features and sensitivity to chemicals are the most important characteristics of terahertz wave. However, how to effectively collect terahertz signal would be a limiting factor. The progress of the detectors is rapid but the devices are still not mature. In the next chapter, several methodologies of terahertz sensors would be discussed. We will introduce our choice - CMOS MOSFET (metal-oxide-semiconductor field-effect transistor) at the end of Chapter 2. 


\section{MOSFET detection}

Research on terahertz detectors is driving the terahertz field to advance, trying to develop sophisticated devices. In this chapter, we will introduce several types of terahertz detectors and then elaborate on MOSFET methodology.

\subsection{Direct and heterodyne $T H z$ detection}

Generally, all the radiation detection systems for $\mathrm{THz}$ could be divided into two categories: incoherent and coherent. Incoherent systems use direct detection sensors, which only collect signal amplitude information and are broadband detection devices; coherent systems utilize heterodyne circuit design which allows not only the amplitude but also phase of the signal to be collected. For the heterodyne circuit design, radiation of high frequency where there are no proper amplifiers is transferred to much lower frequencies and then amplified by low-noise amplifiers. These systems are for narrowband detection [20].

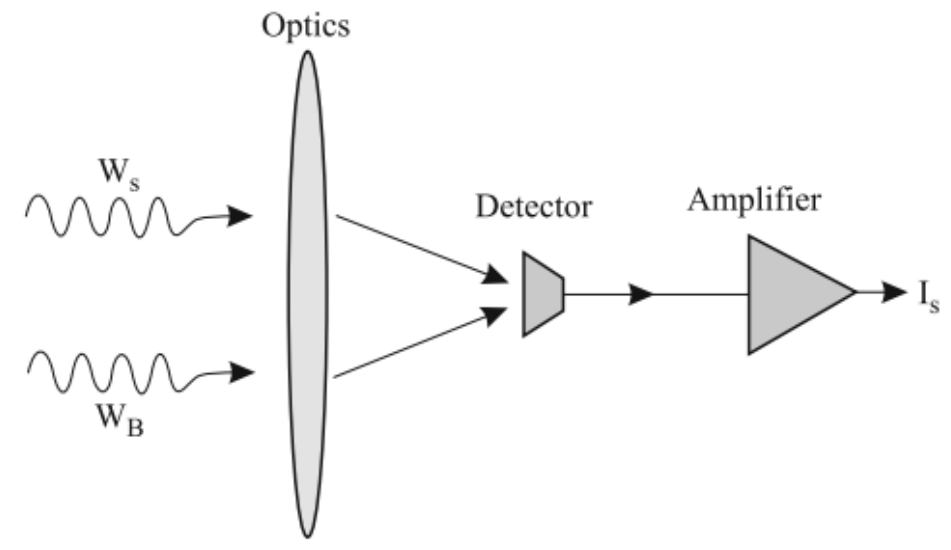

Figure 2.1. Schematic representation of a direct detection. $\mathrm{W}_{\mathrm{S}}$ is the signal power and $\mathrm{W}_{\mathrm{B}}$ is the background radiation power. [20] 
Figure 2.1 shows a schematic diagram for the direct detection. The detector collects both signal and background radiation power. Incident radiation enters the detector via optics and a filter before detector removes the background radiation out of the interested wavelength band. A small signal will be amplified and the resulting Is be further processed.

For direct $\mathrm{THz}$ detection, room temperature detector could be achieved with relatively longer response time and lower sensitivity. However, some cooled semiconductor detectors [20] have much faster response time and have much lower NEP.

The advantage of direct detection systems is its simplicity and the possibility to design large arrays [21-25]. Therefore, most imaging systems use passive direct detection.

In heterodyne detectors, $\mathrm{THz}$ signals are down-converted to intermediate frequency (IF) while the amplitude and phase information of the original radiation are preserved.

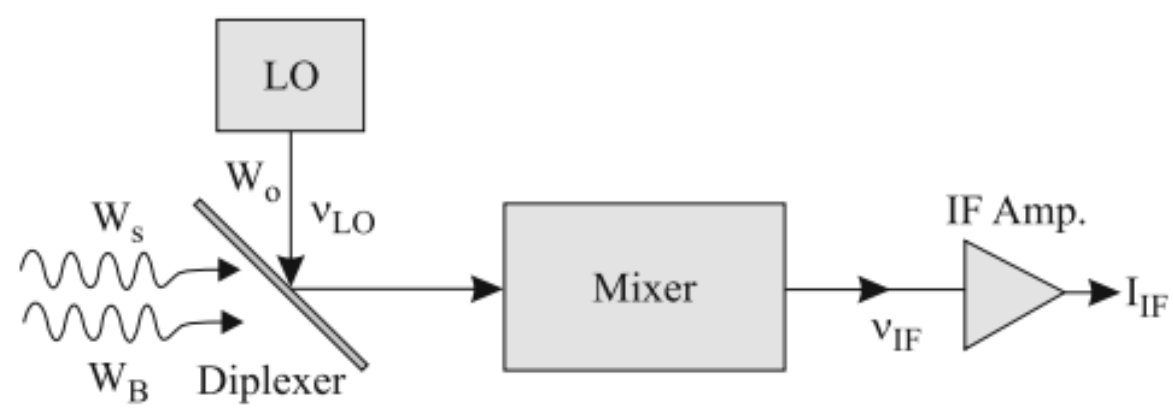

Figure 2.2. Schematic representation of heterodyne detection

In Figure 2.2, there is a radiant power $\mathrm{W}_{\mathrm{LO}}$ (to drive the mixing process) from a local oscillator in addition to the signal and background radiation power. The mixer combines $\mathrm{W}_{\mathrm{S}}$ and $\mathrm{W}_{\mathrm{LO}}$ and generates a signal of intermediate frequency (the difference of signal and local oscillator frequencies). The IF signal is then amplified and processed.

The heterodyne method has advantages over direct detection in that it converts the signal frequency to a lower intermediate frequency, where electronic amplification can take 
place more easily. Also, it has higher spectral resolution. When background radiation is weak, direct detection, operating in wider spectral range, could provide decent resolution. Direct detection is preferable for imaging [26] and can be used in applications where sensitivity is more important than spectral resolution. In active (the scene is illuminated) systems, heterodyne detection could be used to increase sensitivity.

\subsection{Photon and thermal detectors}

Based on the mechanism of detection, terahertz detectors are mainly divided into two groups: photon detectors and thermal detectors.

The thermal effects of incident $\mathrm{THz}$ radiation could be observed through many temperature-dependent phenomena. In Figure 2.3, the detector material temperature changes due to the signal radiation, then the resulting variation of some physical property is used to generate an electrical output. Since the output signal does not depend upon the photonic nature of the incident wave, the thermal effects are frequency independent. The spectral response could be very broad. Three popular thermal detection regimes include bolometer, pyroelectric detector and the Golay cell.

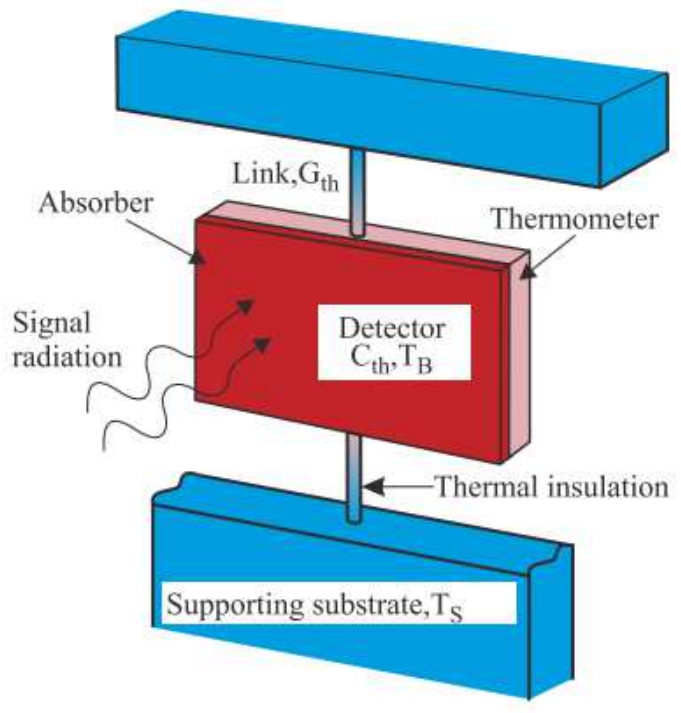

Figure 2.3. Schematic diagram of thermal detector 
The bolometer is a device with a large temperature coefficient so that the incident radiation would induce a large change in resistance. The radiant power produces heat within the material, which in turn creates a resistance variation. There is no direct photonelectron interaction, as in the photoconductor.

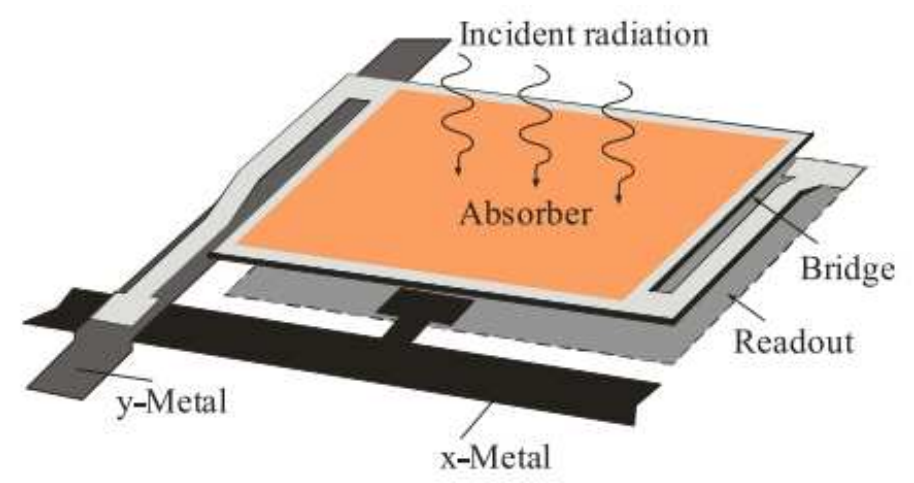

Figure 2.4. Schematic diagram of bolometer

In a pyroelectric detector, the change in temperature modifies the positions of atoms slightly within the crystal structure, so that the polarization of the material changes. This polarization variation results in a voltage across the detector [27].

The Golay cell has a sealed container filled with gas (of low thermal conductivity, for example, xenon). The expansion of gas when heated distorts a flexible membrane on which a mirror is mounted. The mirror's movement deflects a beam of light shining on a photo-resistor. Finally, the output is the change of photo-resistor current [20].

For photon detectors, the incident radiation is absorbed within the material and the photons interact with electrons either bound to lattice atoms, purity atoms or with free electrons. This would cause electronic excitation; the electrical output signals result from the changed electronic energy distribution.

The advantages of photon detection are shorter response time and much higher sensitivity. However, cooling is required in order to reduce thermal noise. Compared to the thermal detection, the response should be within a relatively narrow band. Photoconductive detectors utilize the variation in electrical conductivity resulting from 
change in the number of free carriers generated while photons are absorbed. Photovoltaic detection uses the photon absorption to form a voltage difference across a p-n junction and therefore a photovoltaic current.

Under certain circumstances, MOSFET (Metal Oxide Semiconductor Field Effect Transistor) could be able to serve as a specific kind of photon detector for Terahertz radiation. The characteristics and detection mechanisms of this detection will be illustrated in the next section.

\subsection{MOSFET detection}

First, it is necessary to have a look at some basic concepts regarding MOSFET. MOSFET is one semiconductor device which is generally used as a switch (in the simplest form) or an amplifier of electronic signals. There are four terminals (source, gate, drain and body). Frequently, the body terminal is connected to the source terminal, making this a three terminal device like field effect transistor (firstly patented by Julius Edgar Lilienfeld [28]). MOSFET is the most common transistor and could be manufactured using photolithography.

Figure 2.5 and Figure 2.6 show the schematics of n-MOSFET structure, where $n$ indicates that the source and drain regions are n-type silicon while the substrate is doped with majority positive carriers. The MOSFET works by electronically controlling the width of a channel region along which charge carriers flow (electrons in our case). The channel is formed by applying a voltage at the gate terminal. For example, when a positive voltage is applied to $G$ the holes which carry positive charges in the substrate would be repelled to the ground. Therefore, a depletion region appears near the gate terminal. Also, one inversion layer is close to the insulating oxide, formed by minority carriers attracted to the gate terminal (as indicated in Figure 2.6). Considering the source and drain are both n-type, a channel, where the current flow could go through, is achieved. The charge carriers enter the channel at source and exit via the drain. 


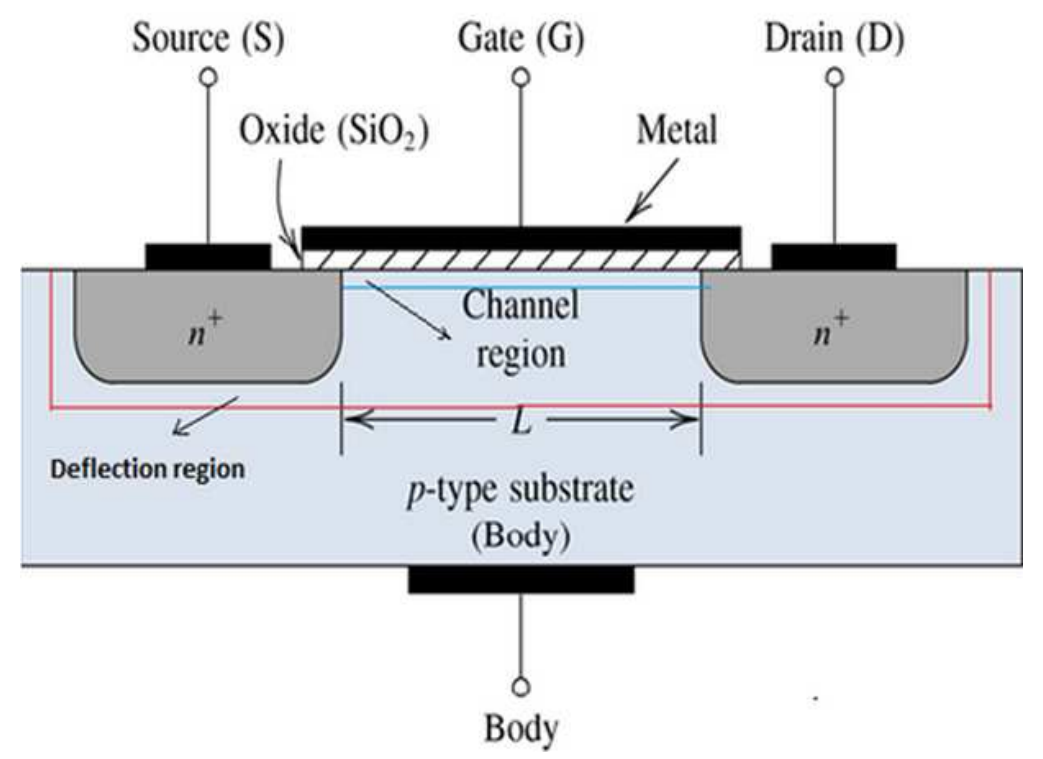

Figure 2.5. n-MOSFET diagram [29]

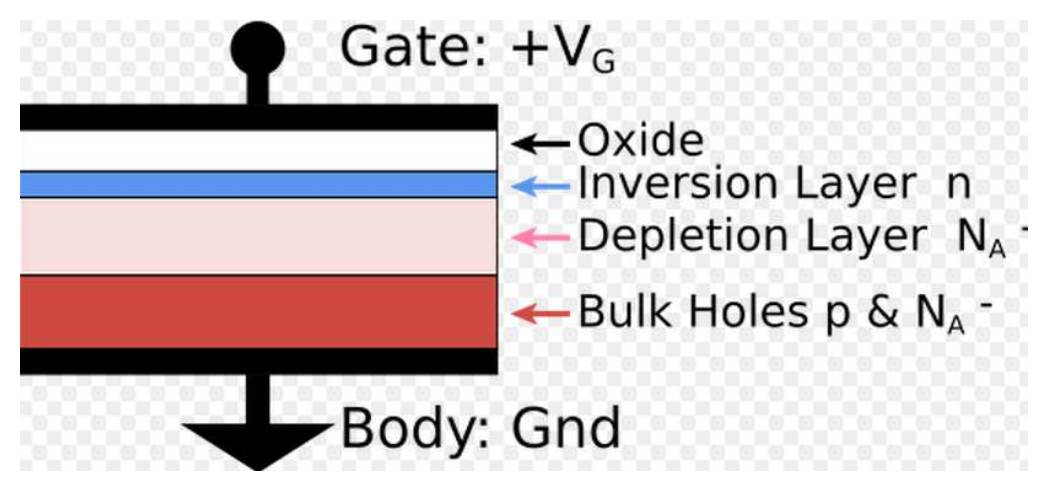

Figure 2.6. Metal-oxide-semiconductor structure on p-type silicon

When there is no gate voltage, it is the Off state; when the gate voltage is above a certain threshold, it is the On state, allowing current to flow between source and drain.

The idea of using MOSFET as a terahertz detector was proposed by Dyakonov and Shur in 1993 [30]. Under appropriate biasing conditions, the electron fluid in the channel could act as a resonator for $\mathrm{THz}$ wave in the form of plasmon waves [20,31]. Plasmonic waves represent rapid oscillations of the electron density in conducting media like plasma or metal. 


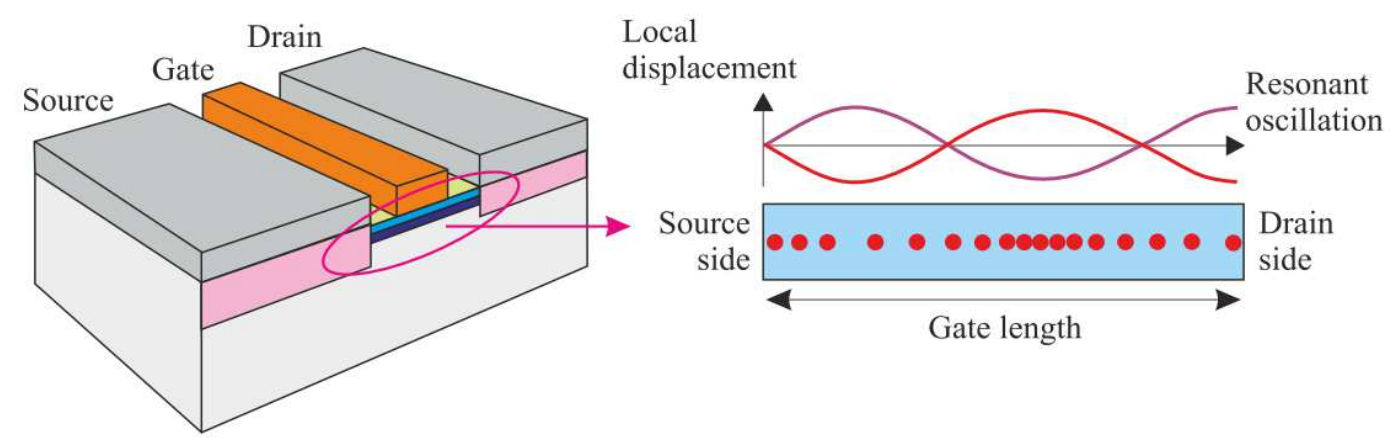

Figure 2.7. Plasma oscillations in MOSFET [20]

Upon proper biasing conditions, incident terahertz radiation would induce an ac signal between the gate and the source terminals. The detection by MOSFET is due to the nonlinear properties of the transistor, leading to the rectification of the ac current. Then, as a result, a photo-response appears in the form of dc voltage between source and drain. Detailed physical mechanism could be referenced to [31]. The resultant voltage is proportional to the radiation intensity, ensuring the validity of this detection methodology.

There are two operation modes for MOSFET terahertz detection: resonant (tuned to a specific wavelength) and non-resonant (broadband). It is directly tunable by varying the gate voltage [32-37]. When the channel length is short enough, a standing plasmon wave could be formed in the channel facilitating signal amplification. In this case, the device has a high responsivity due to the internal amplification and the response is narrow-band. As the channel length becomes longer, the plasmon wave decays before it reaches the other side of the channel. This detector operates in wide temperature range up to room temperature [38,39]. However, at room temperature, electron mobility is too low to support the resonant response to happen [31]. Therefore, in our testing setup, the detection works for the non-resonant mode.

The advantages of using MOSFET terahertz detection include: commercialized CMOS fabrication industry makes the manufacture of low cost and high yield; low NEP for room temperature operation; we could make a very compact detector array to achieve frame 
imaging; lastly, a significant progress in sensitivity could be obtained by introducing an antenna to help couple terahertz radiation to the small absorption element of detector.

Finally, mentioned in Figure 2.9 of [31], a biased voltage between gate and source, Vgs, and also an optimal drain bias current $I_{D}$ could be added to enhance response. The two arms of the antenna are connected to the gate and source for testing.

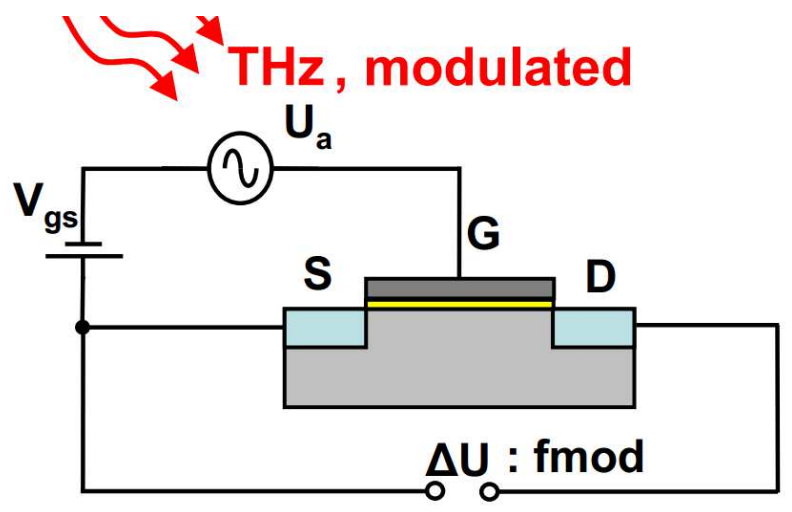

- $\mathrm{V}_{\mathrm{gs}}$ : gate bias

- $U_{a}$ : irradiation induced ac voltage

- $\Delta \mathrm{U}$ : photoresponse

Figure 2.8. MOSFET THz detection with biased voltage, however, the biased current between source and drain is not included

The remainder of the thesis will talk about the work related to the antenna design. 


\section{Numerical method, realization and simulation model}

The subject of this thesis is to simulate terahertz antenna and to optimize the antenna design (part of the work to enhance the signal of the whole detection system) based on the modeling results. This chapter talks about the numerical method used to do simulation, the corresponding software to realize the simulation and details of the model.

\subsection{Finite Difference Time Domain (FDTD) method}

Our numerical simulations are based on FDTD method. Therefore, here we first have a brief review of the FDTD method.

Finite Difference Time Domain is a numerical calculation tool based on Maxwell's equations and is used for modeling computational electrodynamics. The most significant feature of this method is that it is a time domain method; therefore the response results of the simulation could cover a wide range of frequencies with a single run.
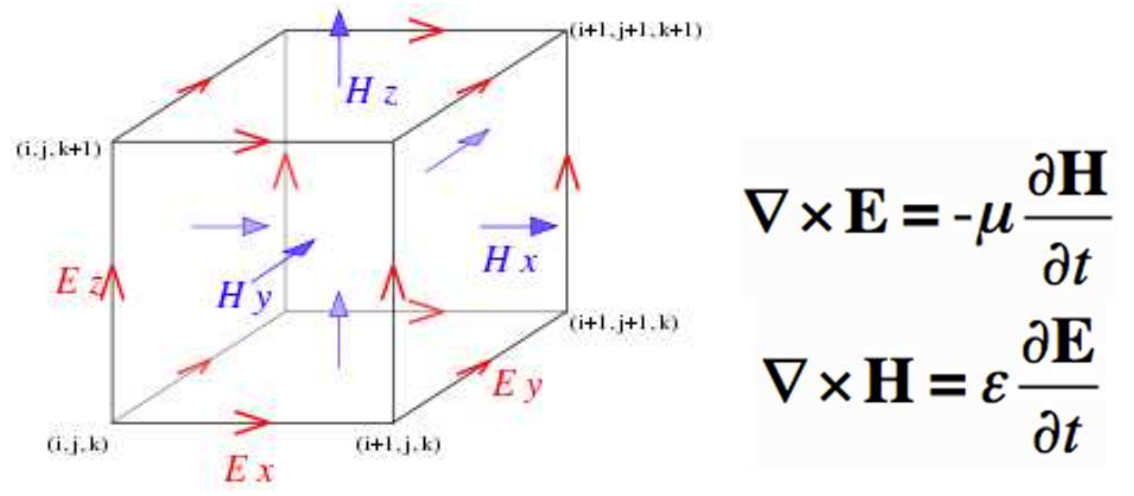

Figure 3.1. Illustration of a standard Cartesian Yee cell in FDTD method (left) and Maxwell's equations which will be discretized (right). It is noted that the electric and magnetic vectors are discretized in space. Also, they are derived alternatively in time. 
FDTD method belongs to grid based calculation techniques (illustrated in Figure 3.1). Maxwell equations are discretized using central-difference approximations to the space and time partial derivatives. Here is an example in vacuum for the plane wave situation (electric and magnetic fields are along $\mathrm{x}$ and $\mathrm{y}$ directions respectively, the wave propagates along $\mathrm{z}$ direction):

$$
\begin{aligned}
& \frac{E_{x}^{n+1 / 2}(k)-E_{x}^{n-1 / 2}(k)}{\mathrm{D} t}=-\frac{1}{e_{0}} \frac{H_{y}^{n}(k+1 / 2)-H_{y}^{n}(k-1 / 2)}{\mathrm{D} x} \\
& \frac{H_{y}^{n+1}(k+1 / 2)-H_{y}^{n}(k+1 / 2)}{\mathrm{D} t}=-\frac{1}{m_{0}} \frac{E_{x}^{n+1 / 2}(k+1)-E_{x}^{n+1 / 2}(k)}{\mathrm{D} x}
\end{aligned}
$$

In these two equations, superscript is time and $\mathrm{n}$ represents a time that is $t=\mathrm{D} t \times n$. The variable in the parenthesis is space and $\mathrm{k}$ represents a location that is $z=\mathrm{D} x \times k$. The two equations assume that $\mathrm{E}$ and $\mathrm{H}$ are separated in both time and space domains. The above equations could be arranged to arrive at the following:

$$
\begin{gathered}
E_{x}^{n+1 / 2}(k)=E_{x}^{n-1 / 2}(k)-\frac{\mathrm{D} t}{e_{0} \rtimes \mathrm{D} x}\left[H_{y}^{n}(k+1 / 2)-H_{y}^{n}(k-1 / 2)\right] \\
H_{y}^{n+1}(k+1 / 2)=H_{y}^{n}(k+1 / 2)-\frac{\mathrm{D} t}{m_{0} \times \mathrm{D} x}\left[E_{x}^{n+1 / 2}(k+1)-E_{x}^{n+1 / 2}(k)\right]
\end{gathered}
$$

This calculation process is clearly illustrated in Figure 3.2: 


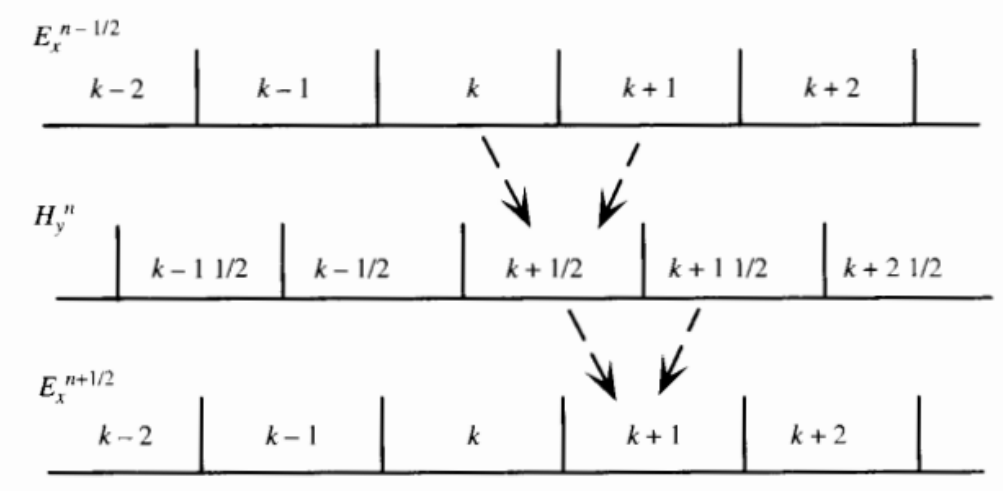

Figure 3.2. 1D FDTD calculation principle

Based on equations (3.3) and (3.4), the $\mathrm{E}$ and $\mathrm{M}$ fields are solved in a leapfrog manner: the electric field vector components and magnetic field vector components of space are derived alternatively. Figure 3.2 shows one time step (completely update EM fields for one time point): for each step, the values of electric and magnetic fields of last step are used to obtain the new values. This process is repeated over and over again until arriving at a steady state. The fundamental and most important idea of FDTD method could be well indicated by this process.

Since FDTD method involves few approximations or simplifications from the starting point of Maxwell equations, it is an accurate and powerful method to predict the electromagnetic behavior of specific structures as long as the index parameters of the materials are known. This technique is very popular in nano-science world and could be well utilized to analyze some terahertz instrumentations.

The weak point is that in order to get acceptable accuracy, the grid must be set to be fine enough to resolve the smallest wavelength and geometrical feature in the model, which substantially increases the calculation burden. Since FDTD requires the entire simulation region to be gridded, the setup of mesh size is an important factor to affect the calculation efficiency. In fact, Lumerical, the software we use, implements a regime to address this issue, which will be mentioned in the next section. Another minor weak point of any 
iterative to mention is the accumulation of errors due to numerical round off and limited precision in calculation.

\subsection{Lumerical FDTD Solutions}

FDTD Solutions is an FDTD-method Maxwell solver package for the design, analysis and optimization of nanophotonic devices, processes and materials. It is developed by Lumerical, a company headquartered in Vancouver, Canada. [40]

This software package helps for rapid prototyping and highly accurate simulations, thus reducing reliance upon costly experimental prototypes, leading to a quicker assessment of design concepts and specific structures. It is now used in various application areas, including fundamental photonics research and industrial applications such as imaging, lighting, biophotonics, photovoltaics. [41]

The latest version of this software is FDTD Solutions 8.9.163 (on Oct 2014). Currently, it supports operating systems of Windows, Mac, Linux and some others. The interface, showed in Figure 3.3, is very user-friendly and most operations are clear and straightforward.

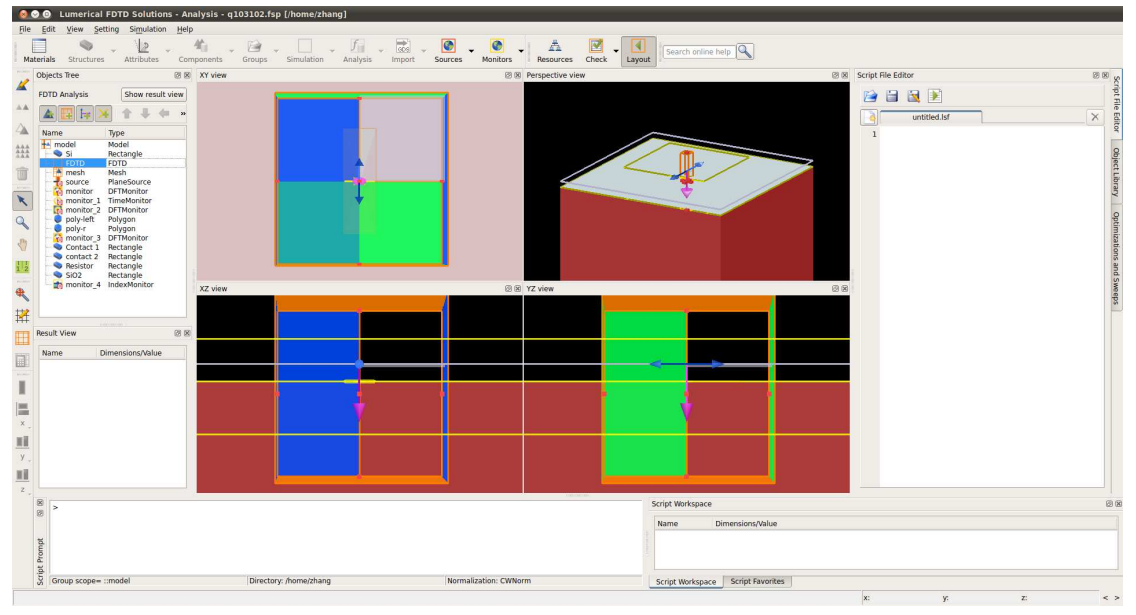

Figure 3.3. The GUI of FDTD Solutions 
The setup modules could be divided into four groups: structures, simulation region, sources and monitors.

The first one, structures, is used to define the physical structure of the research object. This could be done either from internal models or from imported external files. In our cases, we sometimes need to import GDSII (one database file format which is the de facto industry standard for data exchange of integrated circuit or IC layout artwork) files for the antenna design. Importantly, the index data of all materials involved in the simulation should be defined. Several material models and many experimental databases are provided. However, if these are still not satisfying, we can define whatever is desired.

After that, the simulation regions are defined. One general area is mandatory while mesh override regions are optional, there could be more than one override regions. The size, boundary condition, mesh size of the region are the fundamental parameters. Usually, in order to save the required memory while maintaining certain accuracy, several sub-regions could be set to have finer meshes. This is accomplished by setting the override regions. The most common boundary conditions are Perfect Matched Layer-PML (one commonly used type of boundary condition to prevent the radiation going to the boundary from bouncing back to the simulation region), Metal, Periodic, Bloch, symmetric and anti-symmetric. In our simulations, Periodic condition is chosen, which will be discussed later.

The sources part describes the type of incident radiation and related parameters. Different types are dipole, Gaussian, plane wave, total field scattered field and some others. For a plane wave that covers a certain bandwidth, the software optimizes a pulse in time domain to contain all the interested frequencies so that one run in time domain could provide information 
Monitors are for collecting and analyzing data. Two important and straightforward ones are frequency-domain field profile monitor/frequency-domain field and power monitor. The former is used to record the field distribution while the latter could collect information like transmission and reflection through a specific plane.

We feel grateful that we are authorized to utilize research computing resources both from Odyssey high performance cluster at Harvard University and Bluehive2 cluster at University of Rochester. Odyssey has a total of 54000 CPUs, 2140 nodes, 190 TB of RAM, and over $10 \mathrm{~PB}$ of storage [42]. BlueHive2 consists of approximately 200

compute nodes, with 2 x 12-core Intel "Ivy Bridge" CPUs per node and up to 512 GB of memory per node [43]. Both of these two clusters use SLURM as the queue manager.

\subsection{Simulation model}

In this part, details of our model in FDTD Solutions to simulate transmission/absorption are to be illustrated.

The MOSFET detector is small compared to the wavelength of terahertz waves (several microns to hundreds of microns). Therefore, a larger antenna is needed to couple as more incident energy as possible for certain interested spectral bands.

Two types of antennas are considered: bowtie and spiral. Each antenna contains two elements/arms. Examples of their schematics are shown in Figure 3.4. 


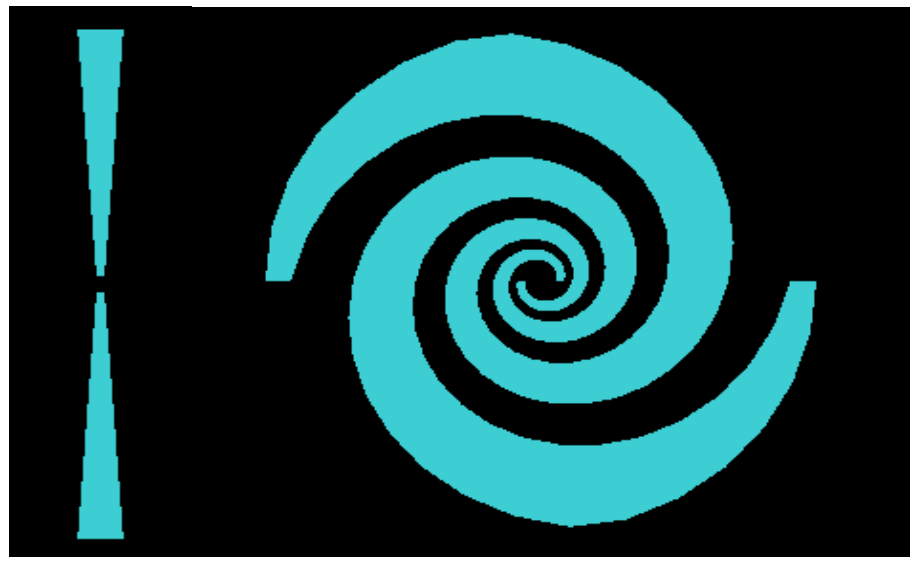

Figure 3.4. Top view of a bowtie antenna (left) and a spiral antenna (right) we try to model (not to scale)

In practice, antennas are made of aluminum and are deposited on a silicon substrate (also an oxide layer may be present, not shown here). For calculating transmission, the whole structure is illustrated in Figure 3.5. A plane wave source propagates from top to bottom. Two power monitors are put above and below the interface in order to collect data of how much energy is reflected or transmitted.

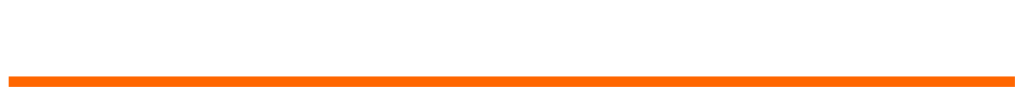

Reflection

Plane wave source

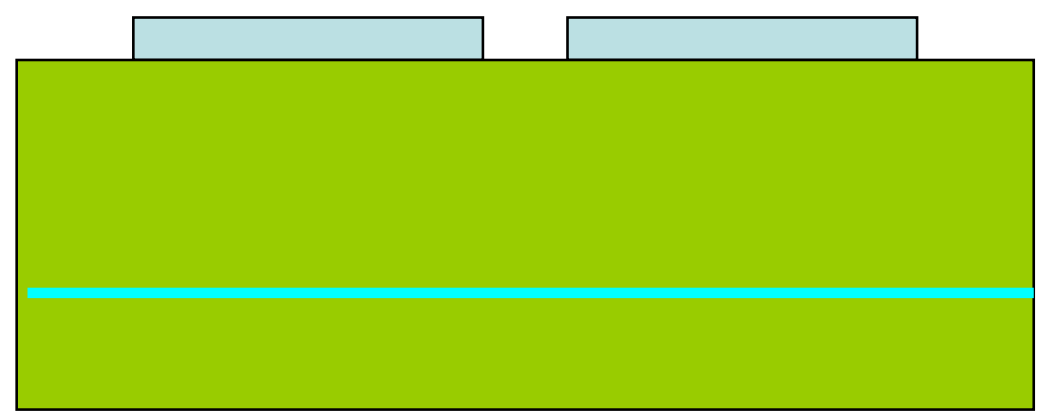

Antenna

Substrate

Transmission

Figure 3.5. Profile of transmission simulation scheme 
For most of our simulations, we used periodic boundary conditions along both $\mathrm{x}$ and $\mathrm{y}$ directions (light propagates along $\mathrm{z}$ direction), including only one unit antenna in the simulation region. This is equivalent to an infinite array of repeating antennas in the $x-y$ plane. For z direction, Perfect Matched Layer (PML) conditions are chosen and distances from boundary to the antenna/substrate interface are at least half of the wavelength of radiation. An override grid region contains the antenna, making the mesh around finer for higher accuracy.

In fact, the transmission monitor should not be in the substrate. However, in order to eliminate the etalon effect of the substrate, the monitor now is placed in the substrate. Some discussion about this fact will be seen in section 4.2.

In the transmission scheme, no element in the model is in charge of absorption considering that aluminum in terahertz range could be approximated as perfect electrical conductor (section 4.1). However, in practice, two arms of the antenna are connected to two terminals of a MOSFET. FDTD is an optical simulation so it could not characterize doped silicon unless we have knowledge of how the doping will affect the complex refractive index of the material. Here, a simple idea is proposed to introduce one absorbing element (Figure 3.6). Two arms of the antenna are connected to a resistor (responsible for absorption) via metal contact. The default set is: the thickness of antenna, metal contact and resistor is one micron. The material of antenna and metal contact is perfect electrical conductor. The resistor is set to be 'conductive' type of material in Lumerical and the resistance could be adjusted by changing its conductivity. For example, for one bowtie antenna, the resistor is a 6 microns long, 1 micron wide and 1 micron thick block with its conductivity set to be $120000(\mathrm{ohm} \cdot \mathrm{m})^{-1}$. Thus its equivalent resistance is equal to a default value, 50 ohms. This value is arbitrarily set (theoretical resistance of a 60-degree infinite bowtie antenna is $60 \mathrm{ohms}$ ) and the issue of the impedance matching will be talked about later. Finally, absorption by the resistor (or the whole system) is derived by one minus transmission and reflection $(\mathrm{A}=1-\mathrm{T}-\mathrm{R})$. 


$$
\text { Absorption }=1-\text { Transmission }- \text { Reflection }
$$
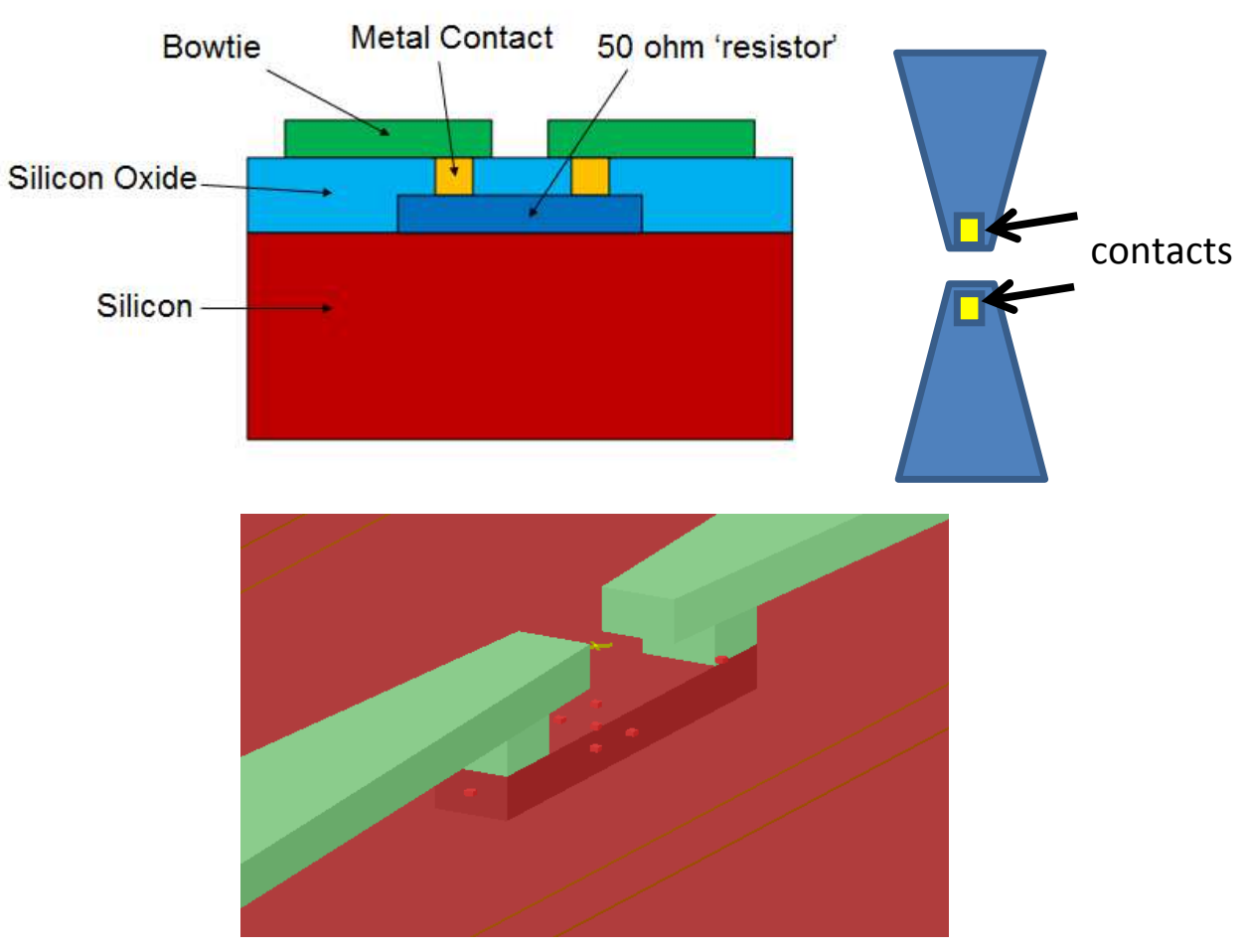

Figure 3.6. Profile and 3D pattern of absorption simulation scheme (not to scale)

While we test various antennas, we may focus on what have already been manufactured. Tiny Imager 4, shown in Figure 3.7, is the first generation chip (manufactured by MOSIS and was under joint project with ITT Exelis and CEIS at University of Rochester) we have, including antenna and the underlying electrical parts. The three bowtie antennas and six spiral antennas were designed by ITT Exelis and the GDS II format designs were loaded in FDTD Solutions for simulation. Each type of antenna has 3-by-3 units and the size of each unit is 100by-100 square microns (Antenna array are densely packed for absorbing more incident radiation). Polarization of incident light is set to be horizontal ( $\mathrm{x}$ direction in Figure 3.7). For convenience, these nine antennas are noted as 'Bowtie antenna 1-3' and 'Spiral antenna 1-6 (actually 1 and 2 are the same one)' in the following content. 


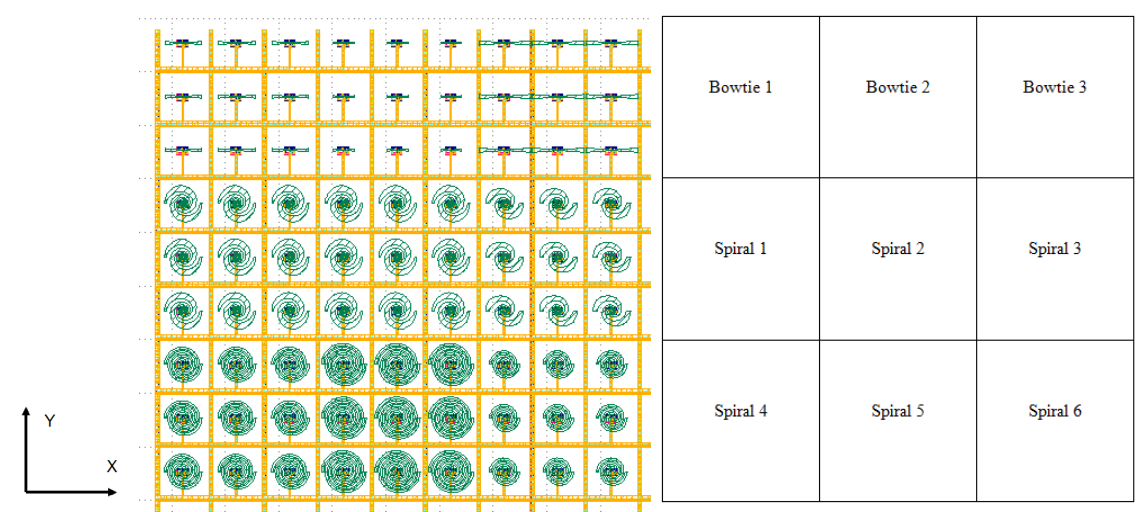

Figure 3.7. Distribution of 9 different antenna designs of Tiny Imager 4. Each type of antenna has 3-by-3 units and the size of each unit is 100-by-100 square microns

The geometric parameters of the bowtie antennas are:

a. Bowtie antenna 1

Inner width: 1 micron; Outer width: 5.7 microns; Gap: 2 microns

Length of one element: 33.2 microns; Total length of one antenna: 68.4 microns

b. Bowtie antenna 2

Inner width: 1 micron; Outer width: 3.3 microns; Gap: 2 microns

Length of one element: 18.8 microns; Total length of the antenna: 39.6 microns

c. Bowtie antenna 3

Inner width: 1 micron; Outer width: 8.1 microns; Gap: 2 microns

Length of one element: 47.6 microns; Total length of the antenna: 97.2 microns
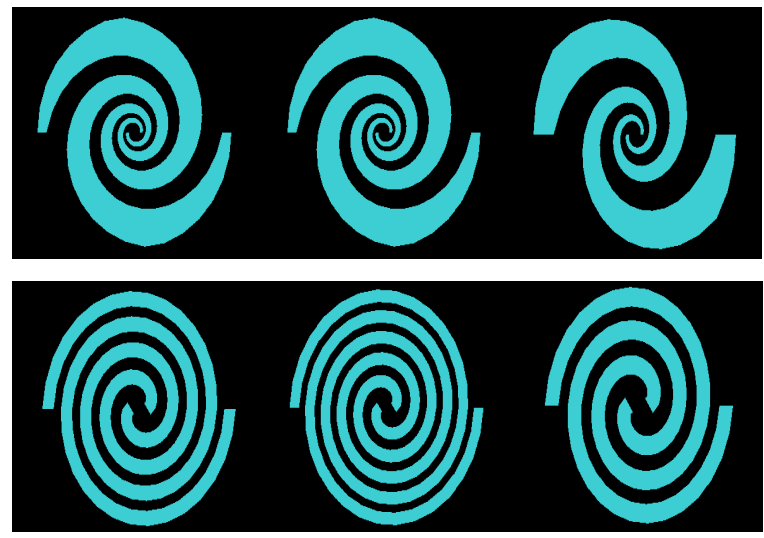

Figure 3.8. Top view of six spiral antennas 1-6 (From top to bottom, left to right, 1 and 2 are actually of the same pattern. Not to scale.) 
The patterns of six spiral antennas are shown in Figure 3.8. The sizes are: For spiral antenna 1 to 4 , the distance from center to one end is roughly 35 microns. For spiral antenna $5 / 6$, it is about $42.5 / 29$ microns.

The practical layout is shown in Figure 3.9, which is much more complicated. Our model is a simplified modeling of the actual pixel (disregarding the underlying gate structure electronics) assuming that the two antenna arms are connected via two PEC contacts to a resistor as illustrated in Figure 3.6.

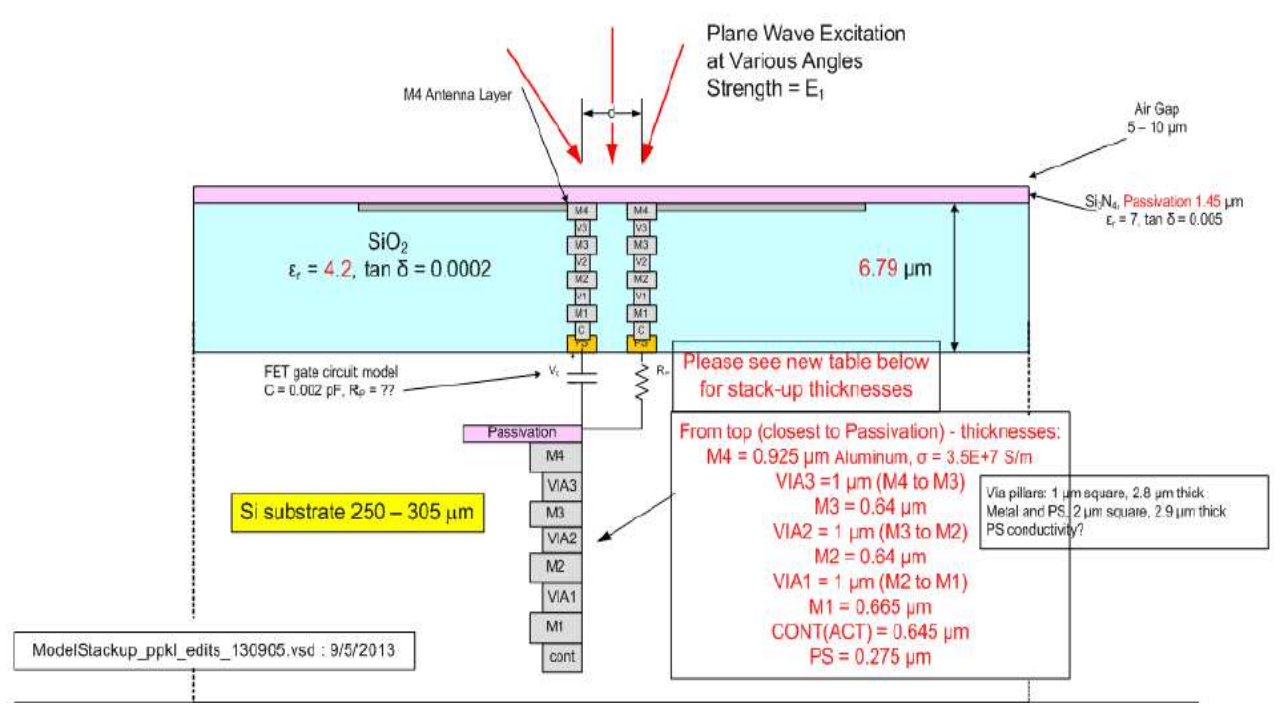

Figure 3.9. Side profile of detailed structure of Tiny Imager 4. The two arms of the antenna are connected to the terminals of MOSFET by several metallic and via layers. The material of via layer is tungsten. The index data in the diagram may not be the actual values.

Chapter 4 and 5 will show the FDTD simulation results of the antenna model discussed above. Firstly, we check the effects of different parameters on the response of the antenna. Then, we apply the knowledge obtained in designing antennas of the second generation chip. 


\section{Effects of different parameters on the antenna performance}

In this chapter, we will execute various tests to check effects of different parameters on the antenna frequency response. The parameters include material index data, pixel density, boundary condition, antenna resistance, antenna shape, etc. Also, some simulation results, like the field distribution would provide better understanding of how the antenna works. Some comparisons between our results and references are made to make sure that our simulation is valid.

\subsection{Material index}

Since the terahertz waveband is a relatively unexplored field, characteristics of the materials in this range have not been well studied or measured compared with those in some other spectral bands. Moreover, choosing an appropriate index model may greatly promote the computational efficiency (for example, as illustrated later in this section, PEC could be used as the material model for the aluminum antenna, which could save much more memory than using the Drude model). In FDTD modeling, index information of every mesh point should be clearly defined. Here, parameters of all the materials used in the simulation are illustrated.

For silicon substrate, data from Grischkowsky et al [44] are used. The silicon samples measured in the reference were of float-zone material (very pure silicon obtained by vertical zone melting) with a resistivity higher than $10 \mathrm{k} \mathrm{ohm}-\mathrm{cm}$. This resistivity is close to the value of undoped silicon. 


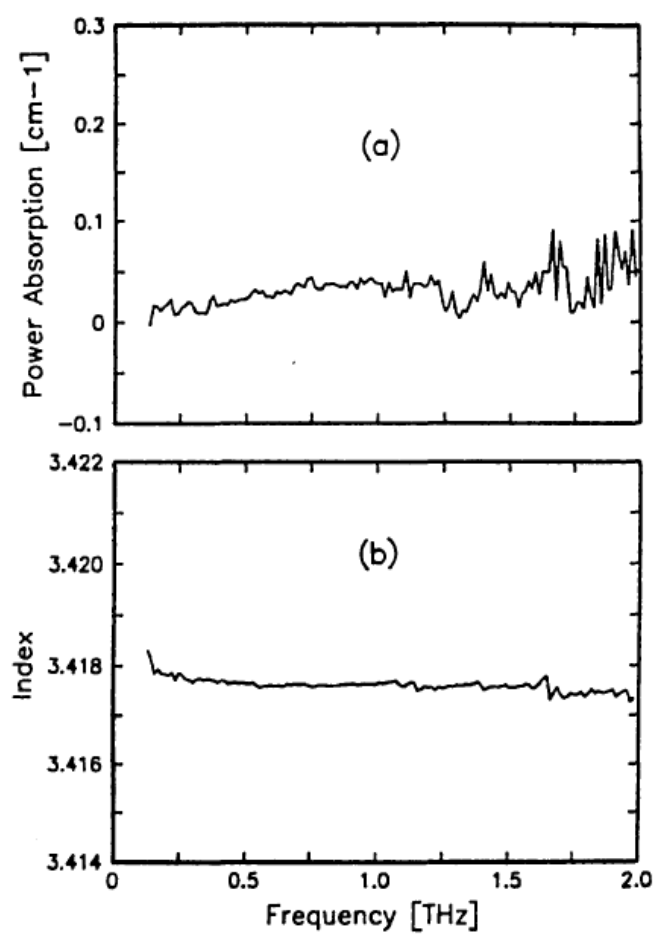

Figure 4.1. Time domain spectroscopy (TDS) measurements of crystalline highresistivity silicon from [44]. (a) Power absorption coefficient, (b) index of refraction Seen from this figure (a/b represent imaginary/real index information), the silicon is almost transparent, together with a remarkably flat dispersion curve. For our simulations, the largest frequency coverage is from 0.1 to $3.0 \mathrm{THz}$. Therefore, after little approximation, the real and imaginary parts of the silicon index were set as 3.4176 and 0 respectively. The corresponding permittivity is 11.7 .

Now we address the index information of silicon oxide. Lumerical internally has experimental data up to 500 microns in wavelength. These data are able to cover the range from 0.5 to $3 \mathrm{THz}$ through multi-coefficient fitting (set the fit tolerance to be 0.01 and max coefficients to be 10). This is indicated in Figure 4.2. However, our simulation range is from 0.1 to $3 \mathrm{THz}$, which is well beyond the range of available experimental data. To address this, two schemes by multi-coefficient fitting and using index values in the design plot (Figure 3.9) from the last chapter (may not be extracted for the terahertz range, permittivity is 4.2 and loss tangent is 0.0002 , real and imaginary parts of index are 2.05 and 0.0002 respectively) were tested. Comparisons between these two choices did not show too much difference for 
transmission/absorption simulations, indicating that the oxide index does not have a significant effect, either method is fine.
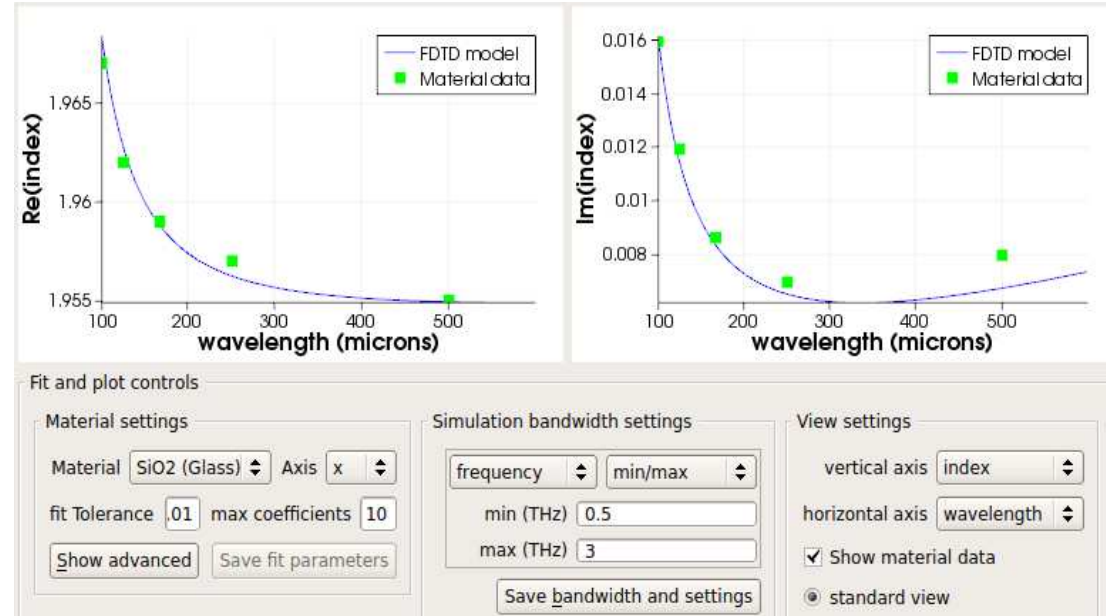

View settings

vertical axis index $\quad$

horizontal axis wavelength $\hat{\imath}$

$\checkmark$ Show material data

- standard view

extended view range

specify view range

Plot in new window Fit and plot

Figure 4.2 . Multi-coefficient fitting for the oxide index data from 0.5 to $3 \mathrm{THz}$

For nitride, our knowledge is from [45]. The real and imaginary parts of the permittivity over the range of $0.4-2 \mathrm{THz}$ are 7.6 and 0.04 , corresponding to index $\mathrm{n}=2.646+\mathrm{i} 0.0066$.

Lastly, how to model the material of the antenna, aluminum, is a most important step. Sun et al [46] experimentally demonstrated that Al-Drude model is an accurate model to fit the real data in the range of 0.5-3 THz. In Lumerical, two parameters are needed to set the Drude model. Here the plasma resonance is $2.243 \mathrm{e}+16 \mathrm{radian} / \mathrm{s}$ and plasma collision is $1.22 \mathrm{e}+14 \mathrm{radian} / \mathrm{s}[47]$. Moreover, in the $\mathrm{MHz} / \mathrm{THz}$ range, we can use a simple PEC model for the metal, aluminum in our case. The skin depth is effectively 0 and can be ignored [48]. Therefore, the Perfect Electrical Conductors approximation was implemented. The simulation results comparison between PEC and AL-Drude could be seen later. Also, Tungsten is another interested model for the antenna. However, the Palik book [49] does not cover the index data of tungsten 
in the range of 0.5-3 THz. What was actually used was derived from a line automatically fitted by FDTD Solutions.

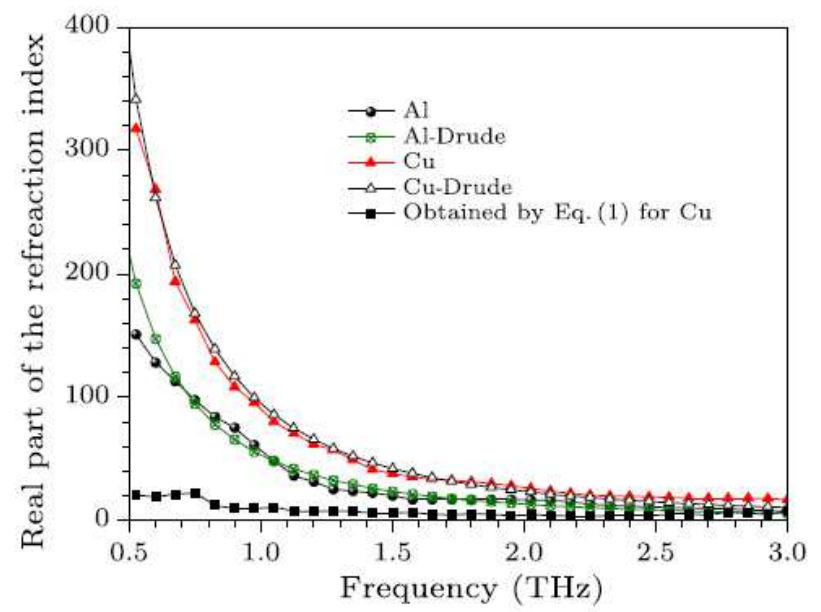

Figure 4.3. Real part of aluminum index information comparison between the experimental data and the Al-Drude model from reference [46]. Imaginary case also agrees well but was not given.

In Figure 4.4, transmission plus reflection equals one anywhere, indicating that there is no absorption by the antenna itself. This should be obvious from the characteristics of the PEC model (it does not absorb incident radiation).
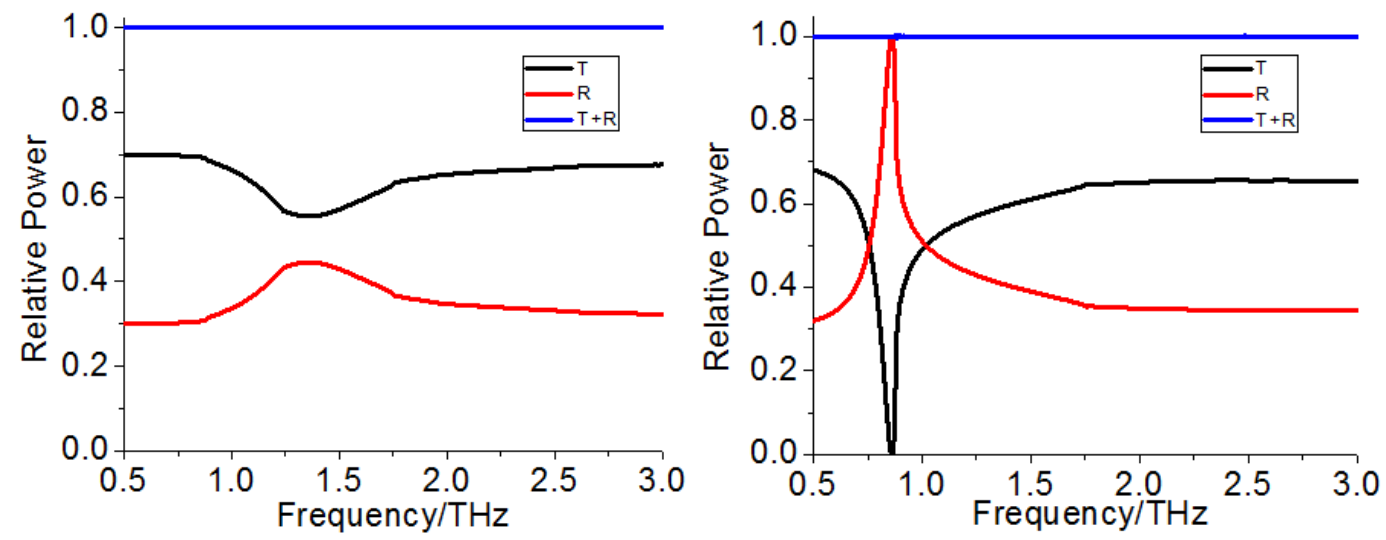

Figure 4.4. Transmission, Reflection and their sum for Bowtie antenna 1 (left) and Bowtie antenna 3 (right) using PEC model 
In Figure 4.5, three models (Perfect electrical conductor, Drude aluminum and Tungsten) for the antenna material are compared together to illustrate how close the simulation results are.
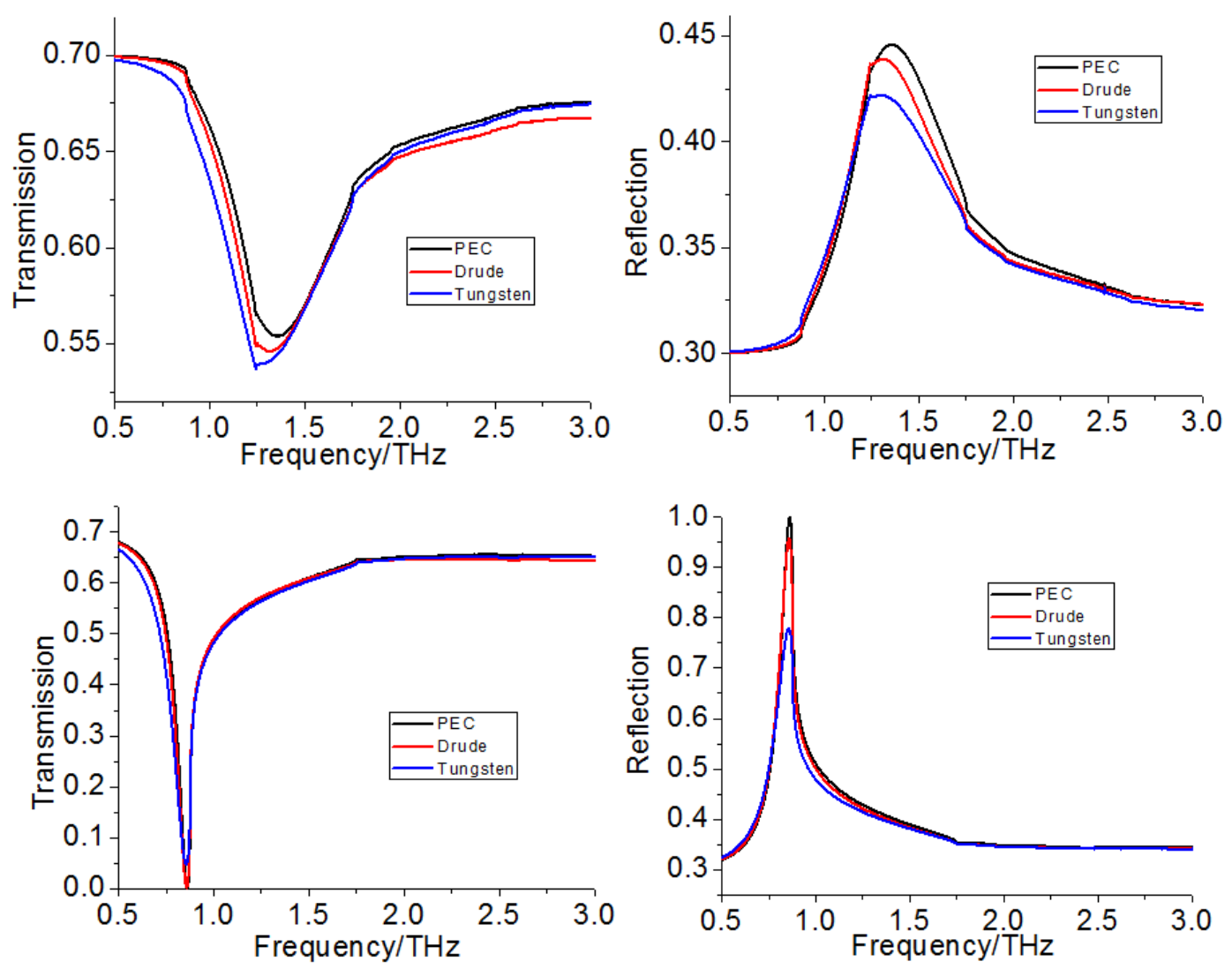

Figure 4.5. Comparisons of transmission (left) and reflection (right) results between the three models for Bowtie antenna 1 (top) and Bowtie antenna 3 (bottom)

The difference among these three models is small. Their curves overlap with each other for a significant portion of the whole range. The results indicate that PEC, which is simple and does not require very fine meshes, is a good model for simulations in this frequency range. Considering that PEC is computationally efficient and is able to get close simulation results with Drude model, therefore, in all the simulations, the material of the terahertz antenna is set to be PEC. Moreover, 
Tungsten is also a good choice for a conductor model when extreme accuracy is not necessary.

\subsection{Comparison of simulation results to existing references}

In order to verify that our simulations using Lumerical FDTD solutions are valid, modeling results are compared to some available data [50]. In this reference, both simulation and experiment were implemented to study the transmission of spiraltype terahertz antennas with square windings.
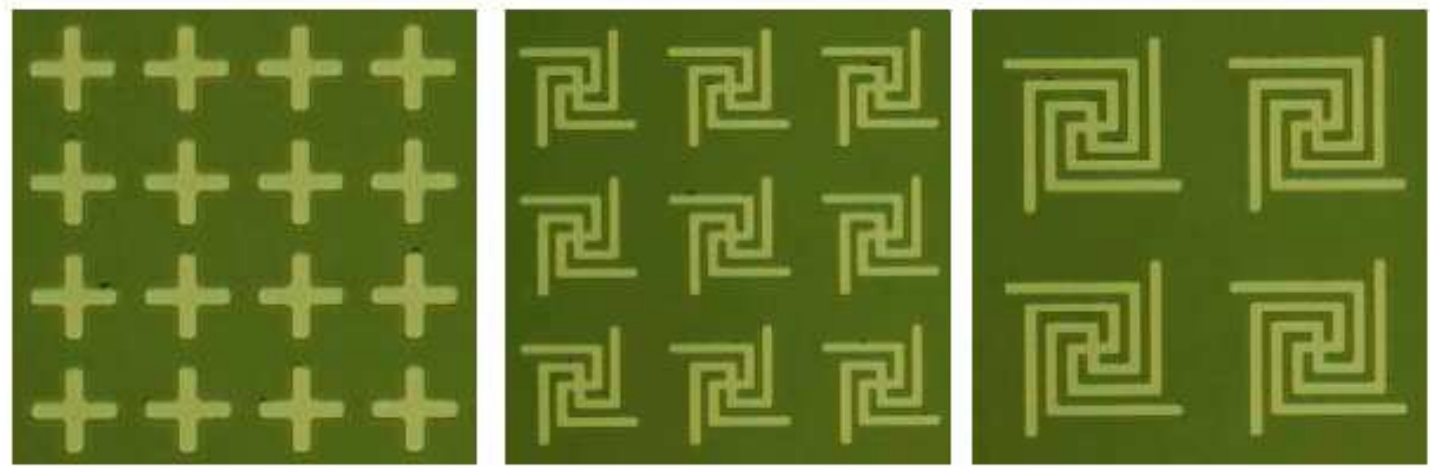

Figure 4.6. Microscopic images of fabricated devices of the spiral type with different winding numbers (From the reference paper [50])
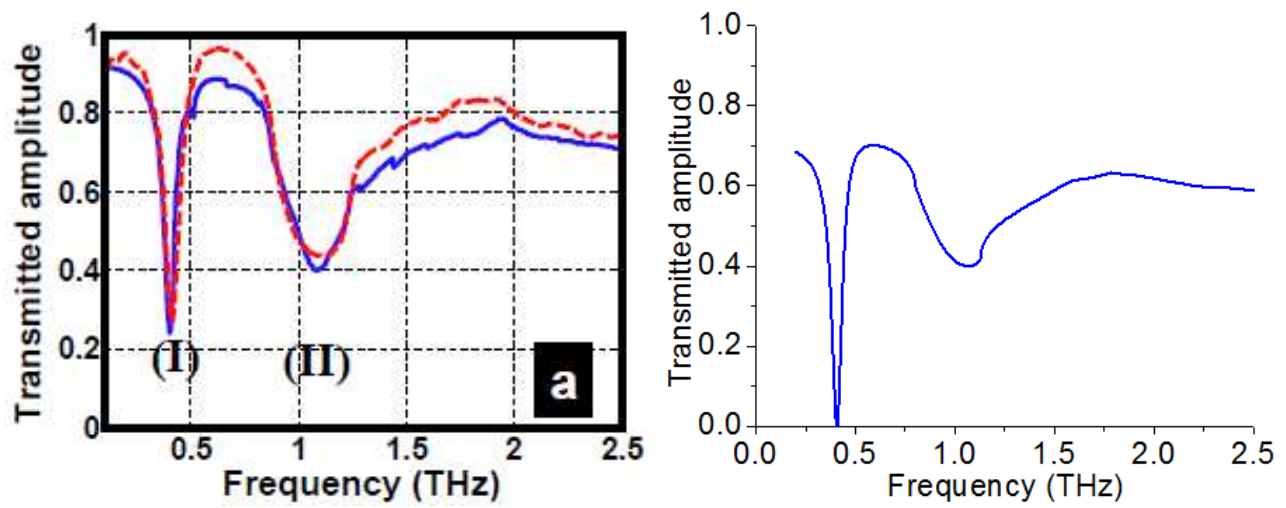

Figure 4.7. Transmission results of spiral antenna with winding number 3 (the second one of Figure 4.6) from the reference (left, blue line is for simulation and red line is for experiment) and from our simulation (right) 
As shown in Figure 4.7, we model the same structures in Lumerical and compare results with the reference. Upon comparison, the conclusions are:

a. For the resonant locations, they match well, the two local minimums occur at about 0.4 and $1.1 \mathrm{THz}$. As winding number increases, there exist more modes, explaining the presence of two dips in the transmission curve;

b. For the transmitted values, there is a 0.2 difference for the base lines from where the curves begin to drop. Our lines start from 0.7 while their lines start from 0.9. Also, the difference of values between the two peaks differs. For the left, it is $0.4-0.2=0.2$ while for the right it is $0.4-0=0.4$.

Possible causes of these differences may be:

a. Simulation methods are different. They use a method called Fourier-model method while our simulation bases on FDTD method;

b. Material parameters. For antenna, they use a Drude model for aluminum and we use Perfect Electrical Conductor (PEC). However, this factor hardly matters. For silicon substrate, their parameters are 0.64-mm-thick, n-type, resistivity $12 \Omega \mathrm{cm}$. In experiment, they normalize the result with bare silicon. For us, the thickness of silicon substrate is set to be infinite and the material is not absorbing;

A simple analysis of the working frequency of a specific dipole or bowtie antenna would indicate that the total length of the antenna is equal to a half wavelength (quarter wavelength is roughly equal to the length of one element of antenna). In this part, some results from references and from our simulations are shown for us to see whether this theory is valid for our models. 


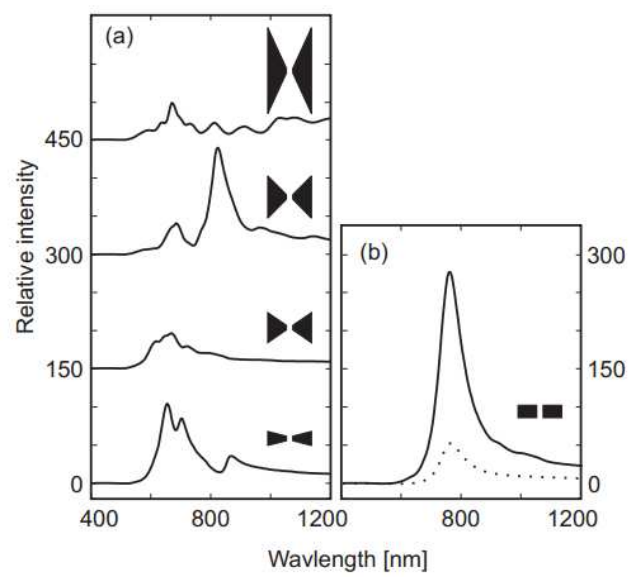

Fig. 2. Relative field intensity spectra in the gap for (a) bowtie and (b) dipole antennas. The dotted line in panel (b) indicates the relative field intensity at the extremity of the dipole antenna. $(\mathrm{l}=230 \mathrm{~nm} ; \mathrm{g}=30 \mathrm{~nm})$

Figure 4.8. Figure 2 from [51], field intensity spectra in the gap of antennas

Field intensity spectra in the gap of bowtie/dipole antennas are calculated in [51], the resonant frequency could be seen. In the right Figure 4.8, the total length $\mathrm{L}$ is $230 \mathrm{~nm}$, so the resonance should be at around $2 \times 230=460 \mathrm{~nm}$ according to the half wave dipole hypothesis. However, the results shown above are around $800 \mathrm{~nm}$.

Another example is from [50], where the transmission of a cross antenna is calculated. Although this is a cross antenna, it could be approximated as a rectangular bar/dipole due to the vertical polarization of the incident light. This bar is 30 microns long. Since $2 \times 30=60$ microns, the predicted resonant frequency is 5 THz. However, their simulation result is a little less than $2 \mathrm{THz}$.
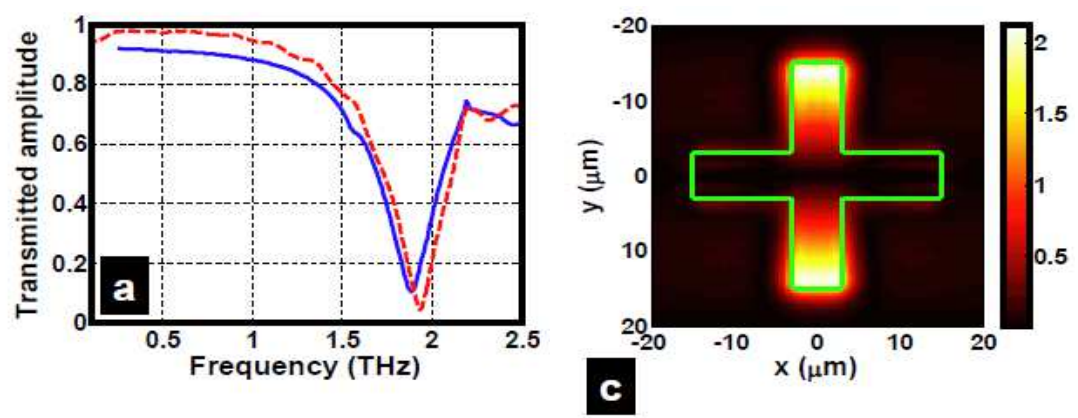

Figure 4.9. Transmission curve of cross antenna for vertical polarization from [50] 
Now, we turn to have a look at our own results. In Figure 4.10, for three bowtie antennas, the transmission and absorption results are shown. Bowtie antenna has much better bandwidth than a thin-wire dipole antenna (considering that antennas with more volume have wider bandwidth) and is easy to construct. Therefore, bowtie antenna is selected as the subject of our simulations.
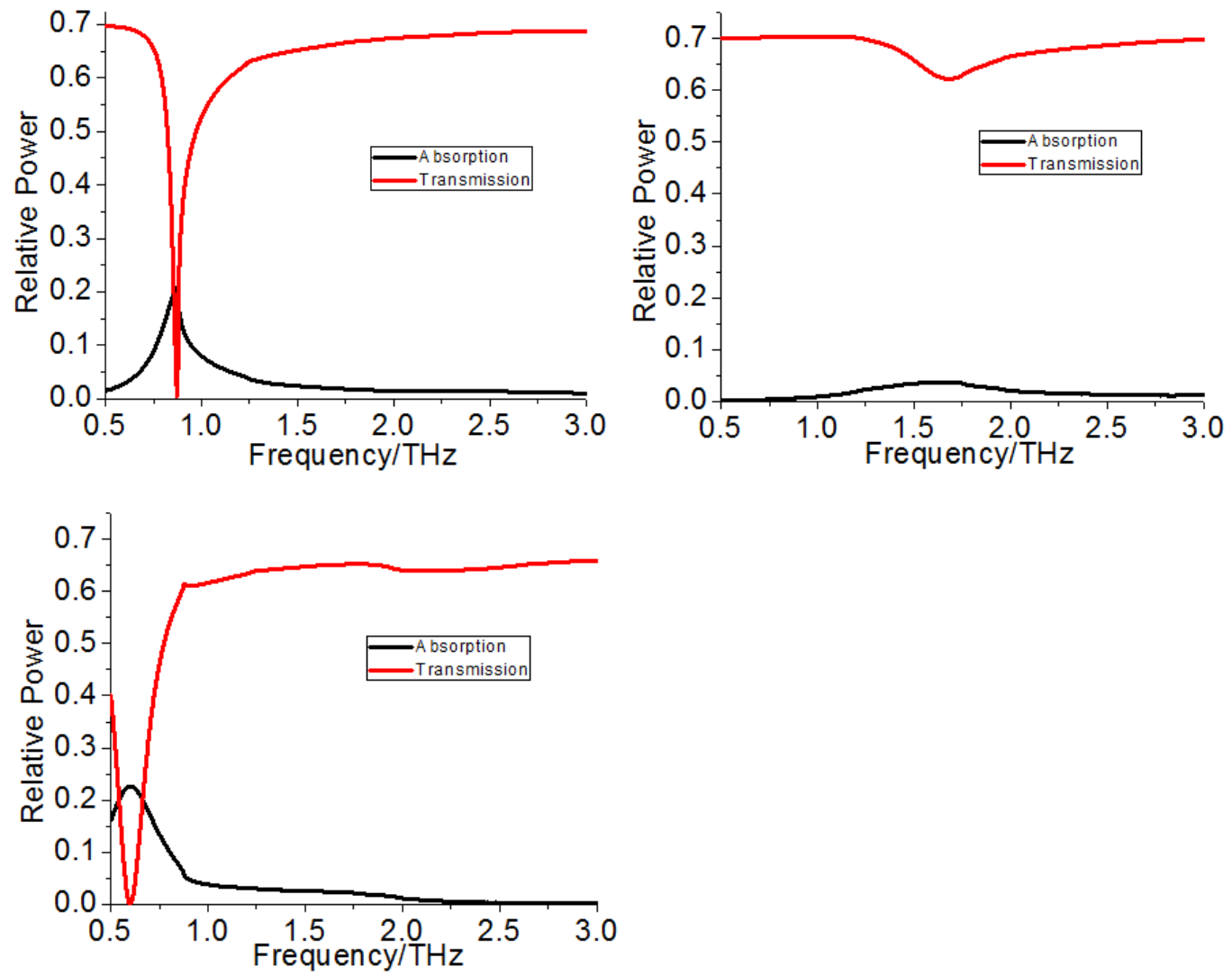

Figure 4.10. Transmission (red) and absorption (black) curves of three bowtie antennas (top left for Bowtie antenna 1, top right for Bowtie antenna 2, bottom left for Bowtie antenna 3) with unit pixel to be 100-by-100 square microns

The expected resonant wavelengths of the 3 bowtie antennas are 136.8, 79.2 and 194.4 microns, respectively, corresponding to frequencies of 2.2, 3.8 and 1.5 THz. However, simulation results show that the transmission peak frequencies are much smaller (about $0.875,1.725$ and $0.625 \mathrm{THz}$ ) than the values that the theory would expect. This conclusion is in agreement with the results in the two references, the 
simulation frequency results are lower than the theoretic expectations. The half wavelength theory is only valid for very thin and standalone dipole antennas [52]. For our cases, the antenna is sitting on the silicon substrate. We shall expect that the substrate would affect the frequency response of the antenna as compared to standalone antennas.

\subsection{Effect of antenna pixel pitch}

In this part, the effect of the spacing between adjacent antennas (antenna pixel pitch) is studied. First, the transmission curves of cases with different periods are calculated. Intuitively, smaller period (more compact antenna array) would grant the antenna array more power to control the incident light. As the spacing approaches, the 'fill factor' gets higher and the array intercepts more EM energy. Curves in Figure 4.11 will verify this prediction.

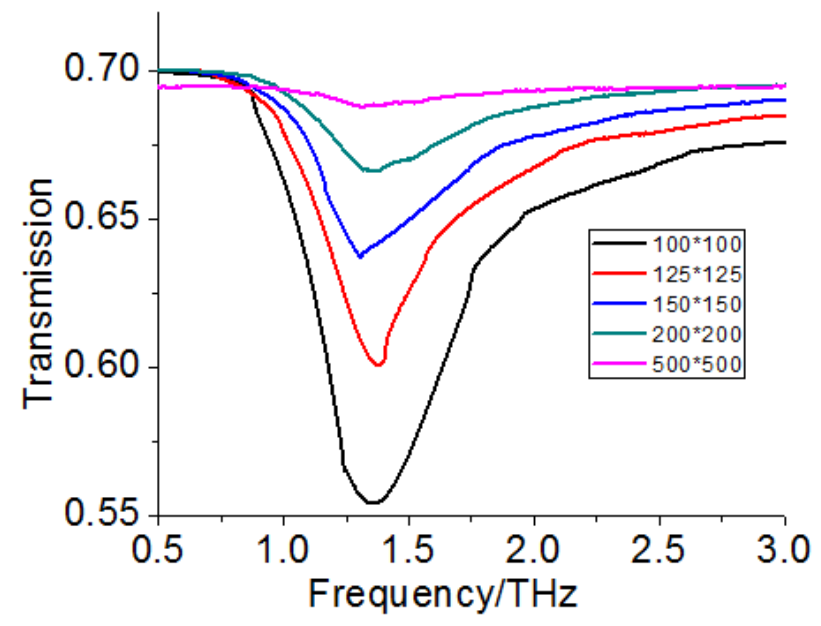

Figure 4.11. Transmission curves of Bowtie antenna 1 with different periods from 100 to 500 microns (horizontal and vertical periods are the same)

It could be seen that as the spacing between adjacent antennas becomes larger more light could transmit the system at resonant frequency, meaning less power to control the incident energy. It is seen that lower pixel pitch provides increased efficiency (lower 
transmission). Therefore, to get higher system efficiency, it is a good idea to make densely packed antenna arrays.
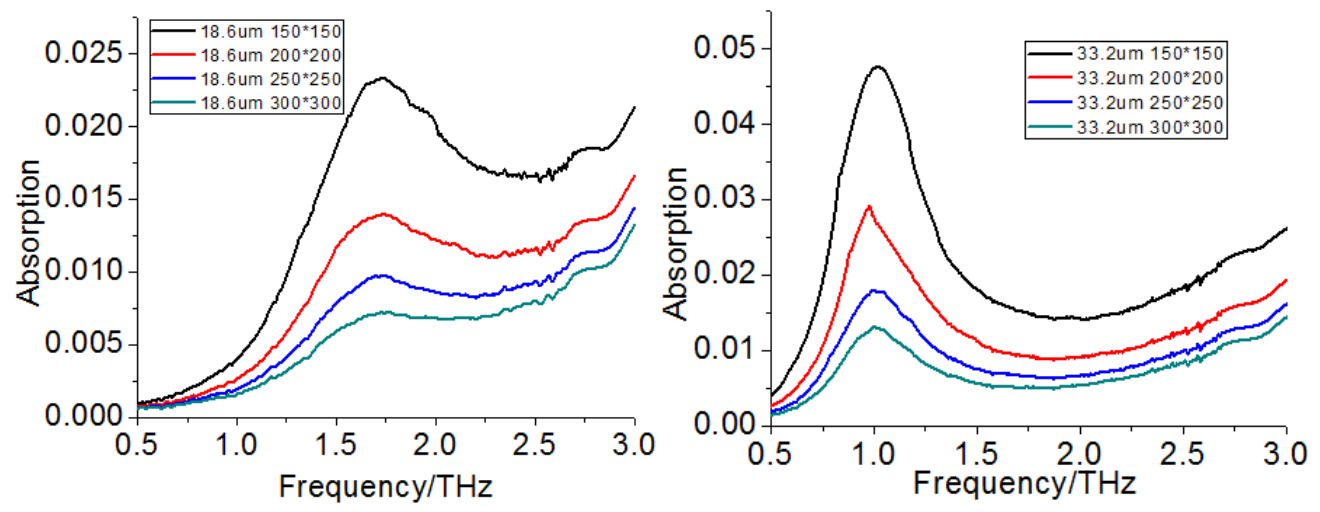

Figure 4.12. Absorption curves for two bowtie antennas (both with a 50-ohm resistor) of different periods. The unit of period is micron. The inner and outer widths of antennas are 1 and 3.1 microns. They have a 2-micron gap, the length of one arm are 18.6 and 33.2 microns respectively.

The effect of pixel pitch could also be obviously seen by exploring the absorption. Absorption represents the percentage of incident energy that is absorbed by the antenna/detector. One most important conclusion of our simulations is that the frequency location of peak absorption does not change when the spacing between adjacent antennas alters. In Figure 4.12, it is observed that peaks of the curves keep fixed for both these two sets of bowtie antenna arrays, around 1.7 THz for the 18.6micron antennas and $1.0 \mathrm{THz}$ for the 33.2-micron antennas.

Numerically, it is noted that the peak absorption values of antenna with a period of $150 * 150$ microns is around four times of the peak values of antenna with a period of $300 * 300$ microns. Since the former case has four times antennas in number as the latter case, it could be summarized that the absolute absorption for a single antenna is fixed. Therefore, in order to absorb as more energy as possible, it is desired to have more antennas in the unit area, which means densely packed array should be considered favorable. 


\subsection{Broadband bowtie antenna}

Figure 4.13 shows a copy of the antenna design given in $[53,54]$, demonstrating that bowtie antennas with wider taper could cover a larger range of wavelengths. Some tests have been made to explore the frequency characteristics of broadband bowtie antennas.

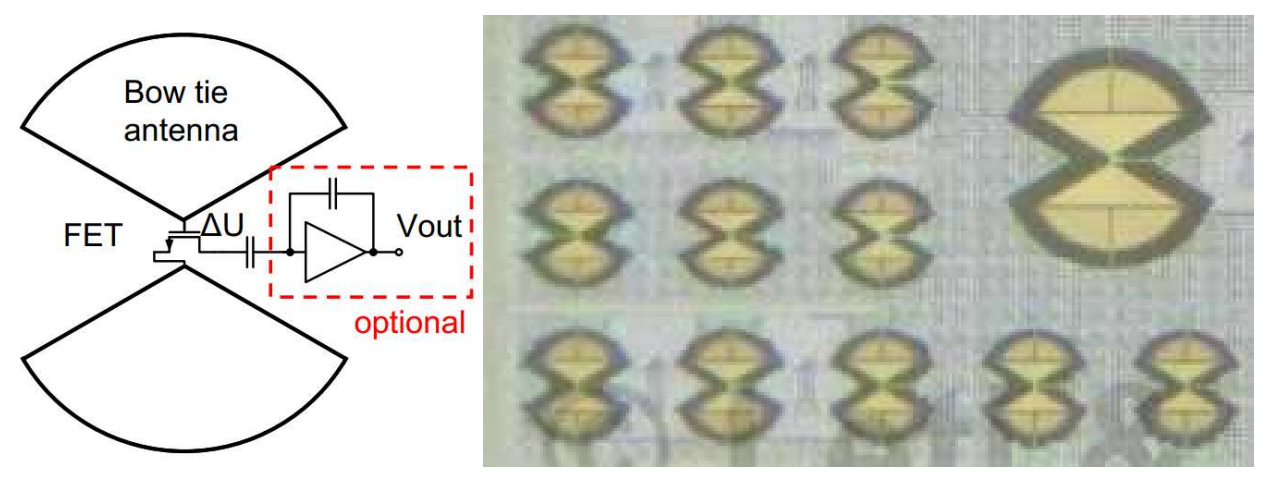

Figure 4.13. Sketch of the Bowtie antenna mentioned in the report of the French group [53]

Here, the characteristics of the fat bowtie antennas with rounded edges are examined.

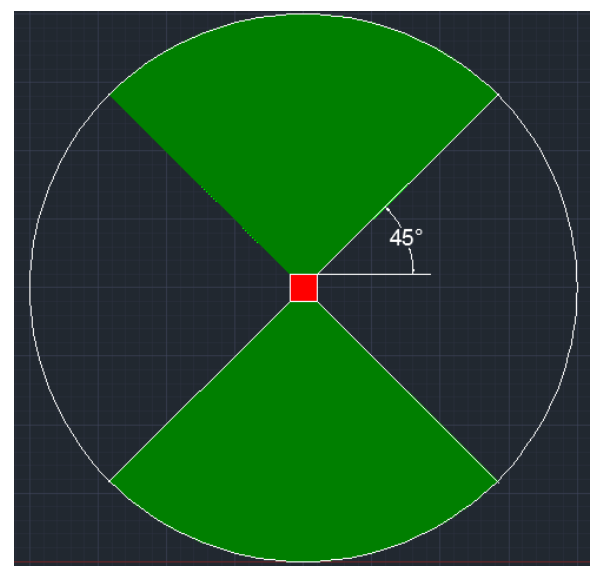

Figure 4.14. Schematic of Fat Bowtie antenna modeled here

Our design is shown in Figure 4.14, where the fat bowtie antenna is restricted within a circle. The red part ( $10 * 10$ square microns) is a 50 -ohm (arbitrarily set) resistor. For one set of antennas with one specific radius, the angle in the figure (45 degrees here) is 
changed from 55 to 25 degrees in increments of 10 degrees. Therefore the smaller the angle is the fatter the antenna is. In our simulations, three sets of fat bowties (of different radii) are included and detailed parameters are listed:

Set 1: Diameter is 150 microns, pixel pitch is $200 * 200$ square microns;

Set 2: Diameter is 200 microns, pixel pitch is $250 * 250$ square microns;

Set 3: Diameter is 250 microns, pixel pitch is $300 * 300$ square microns.

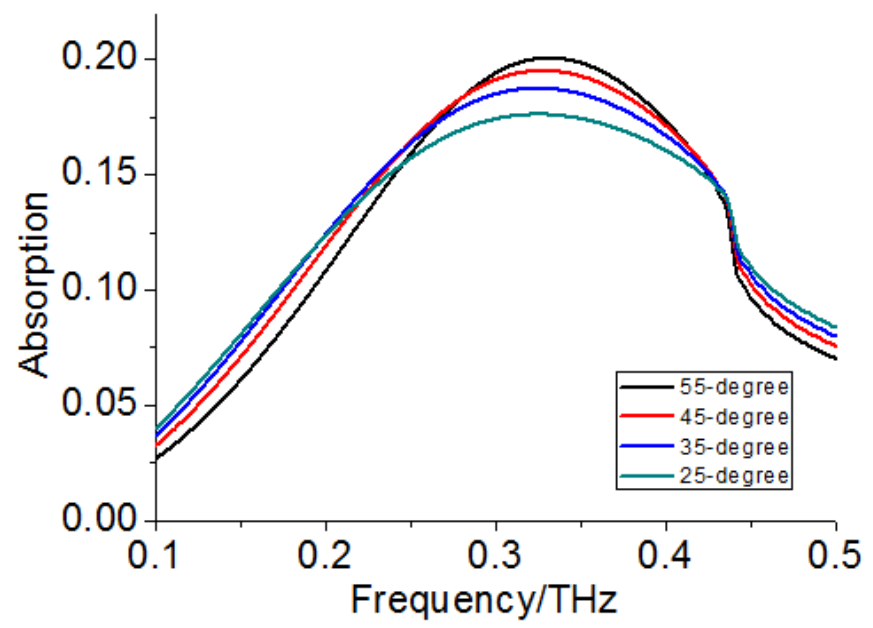

Figure 4.15. Absorption curves for fat bowtie antennas restricted within a circle of which diameter is 150 microns

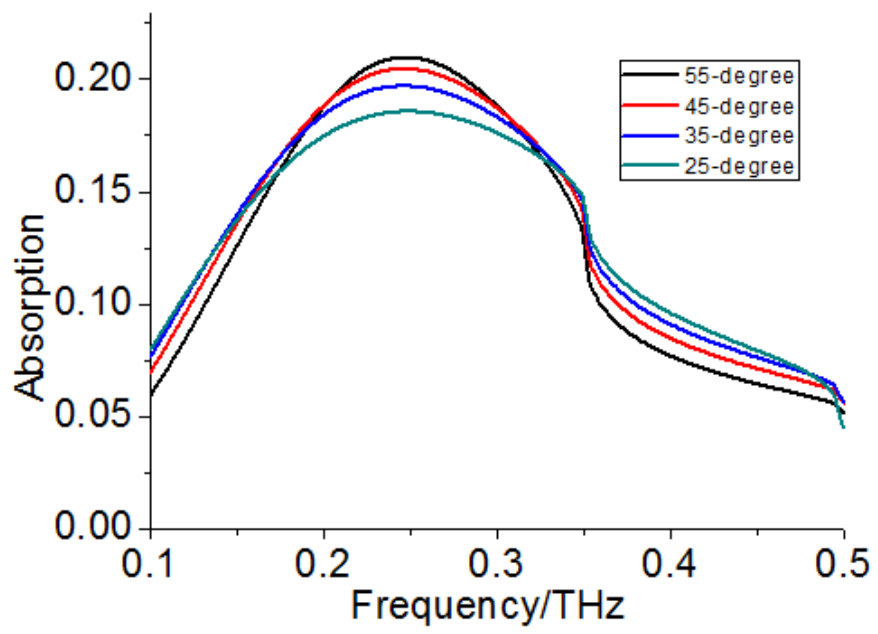

Figure 4.16. Absorption curves for fat bowtie antennas restricted within a circle of which diameter is 200 microns 


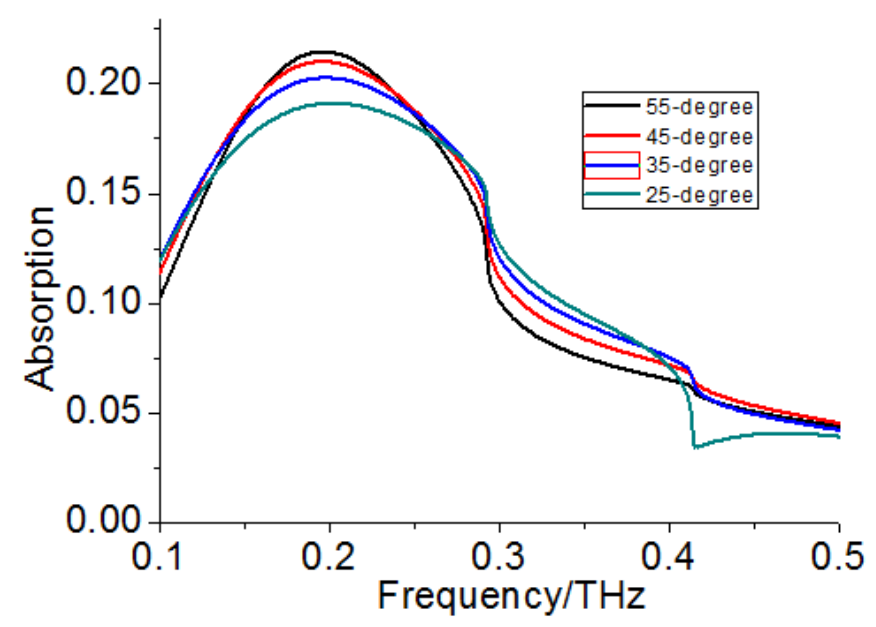

Figure 4.17. Absorption curves for fat bowtie antennas restricted within a circle of which diameter is 250 microns

From the above three sets of curves, Figure 4.15-4.17, it is seen that for a specific diameter the resonant frequency stays the same no matter what the included angle of the antenna is. Actually, this is an expected conclusion since the longest distance between two points within the antenna keeps the same for a set of a specific diameter. In Table 4.1, the values are illustrated.

\begin{tabular}{|c|c|c|}
\hline Antenna Diameter/micron & Resonant frequency/THz & Resonant wavelength/micron \\
\hline 150 & 0.33 & 910 \\
\hline 200 & 0.25 & 1200 \\
\hline 250 & 0.20 & 1500 \\
\hline
\end{tabular}

Table 4.1. Resonant frequency/wavelength information for fat bowties of three different diameters 


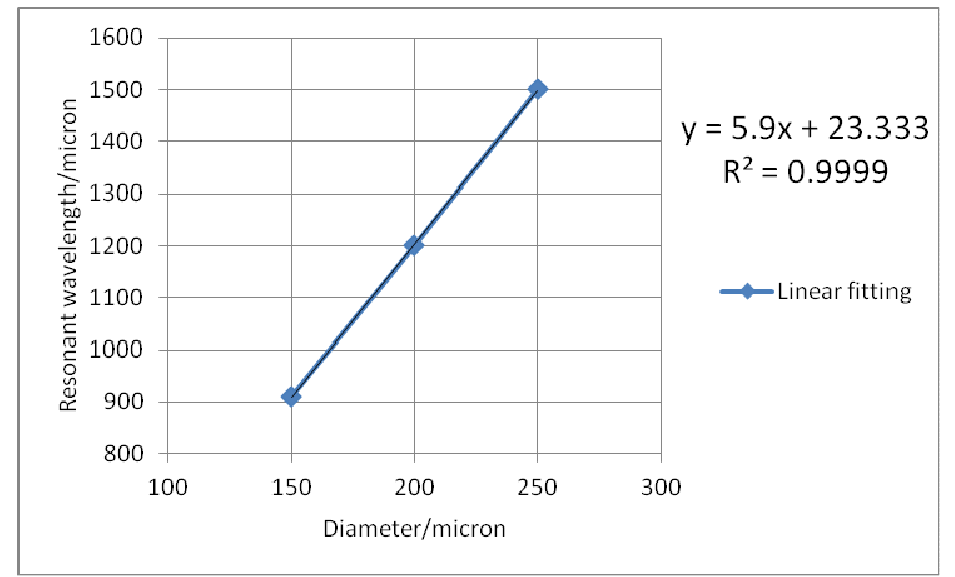

Figure 4.18. Linear equation fitting between the antenna diameter and resonant wavelength. $\mathrm{x}$ is diameter of the fat bowtie antennas and $\mathrm{y}$ is the corresponding resonant wavelengths.

As the diameter increases (antenna becomes longer), the resonant location shifts to lower frequency. From the above table, we try to fit a linear relationship between the Diameter of the antenna and the corresponding resonant wavelength. The result shows that the relationship is very close to be linear, which is as expected and reasonable. This feature should be different for the fat bowties with sharp edges, not rounded. This could be seen in the next chapter.

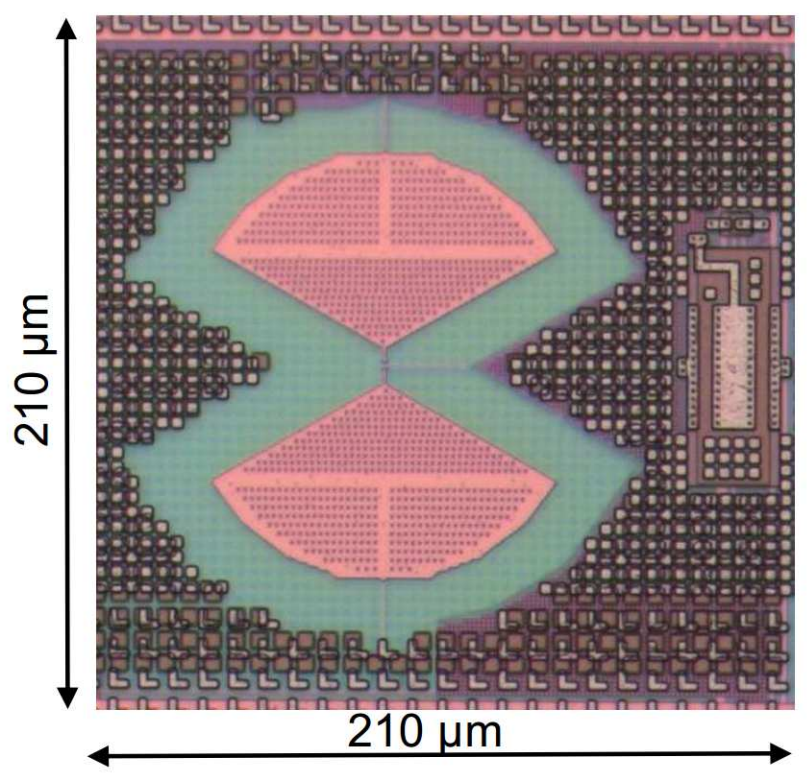

Figure 4.19. Pixel photograph of French group design [53] 
The validity of the fitting equation in Figure 4.18 could be roughly checked by looking at the design of French group. In Figure 4.19, the diameter of the fat bowtie antenna (works at $300 \mathrm{GHz}$ ) with rounded edges is about 150 microns. The calculated resonant wavelength for antenna with a 150-micron diameter should be 908 microns from the equation in Figure 4.18. Considering that $300 \mathrm{GHz}$ corresponds to 900 microns, this prediction is very accurate.

Another notable feature is that as the angle becomes smaller (the antenna covers a larger area), the peak value drops down a little. However, the absorption curve becomes wider, which is reasonable for a fatter antenna.

\subsection{Polarization}

Here different incident polarizations are considered. The antenna subject is the fat bowtie whose diameter is 150 microns and the angle is 65 degrees (used in the last section). The orientation of the antenna is along y axis (vertical). Four different directions of the incident light are illustrated here (For the UL-LR and UR-LL cases, they are 45 degrees to the vertical or horizontal directions):

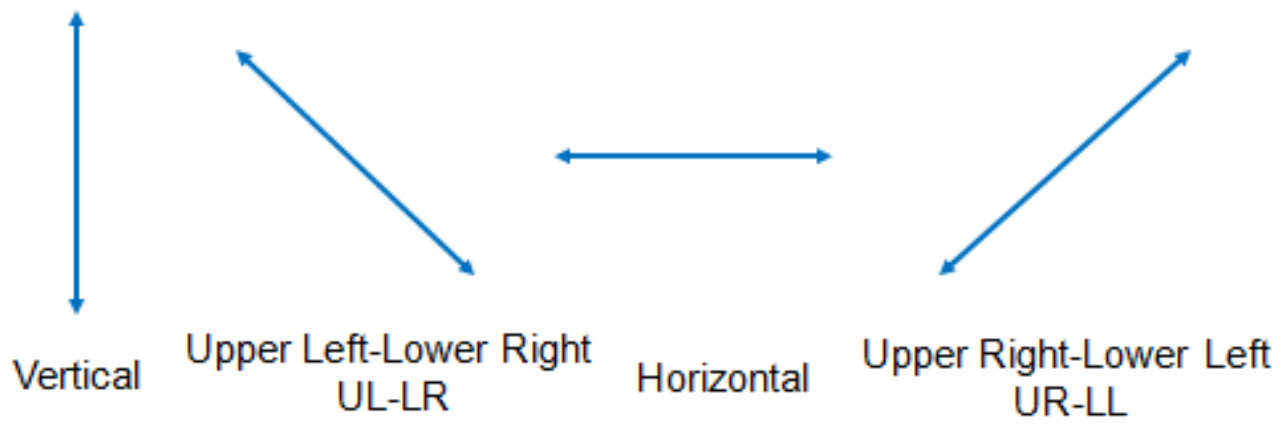

Figure 4.20. Four different incident polarizations 


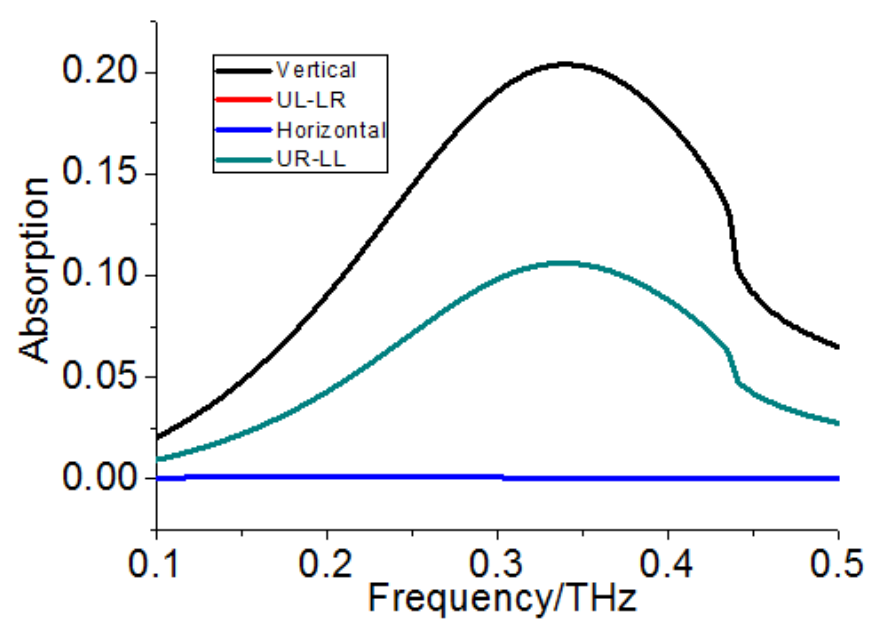

Figure 4.21. Absorption curves of the fat bowtie antenna for different polarizations

Since the antenna is oriented along the vertical direction (y axis), the response should be maximum when the incident light is also vertical while there is no absorption for the horizontal polarization. These theories are well verified from Figure 4.21. More importantly, for the 45-degree and 135-degree cases, the absorption values are half of the vertical polarization's (These two curves overlap with each other so we can not see the red line). This conclusion makes sense when considering that the 45-degree incident light could be equally decomposed into the vertical and horizontal components. There is no absorption for the horizontal light so that the entire response is half of the original value.

\subsection{Periodic boundary}

First, we did an experiment to compare results of two cases for Bowtie antenna 1: 1. Reduce the horizontal period to be half of its original value (from 100 microns to 50 microns while keeping the vertical period still to be 100 microns); 2. Fill the original one unit with two antennas with a horizontal spacing of 50 microns. Considering the characteristic of periodic boundary condition, the results should be the same or with very little difference. The following figures, 4.22 and 4.23 , verify this fact. 

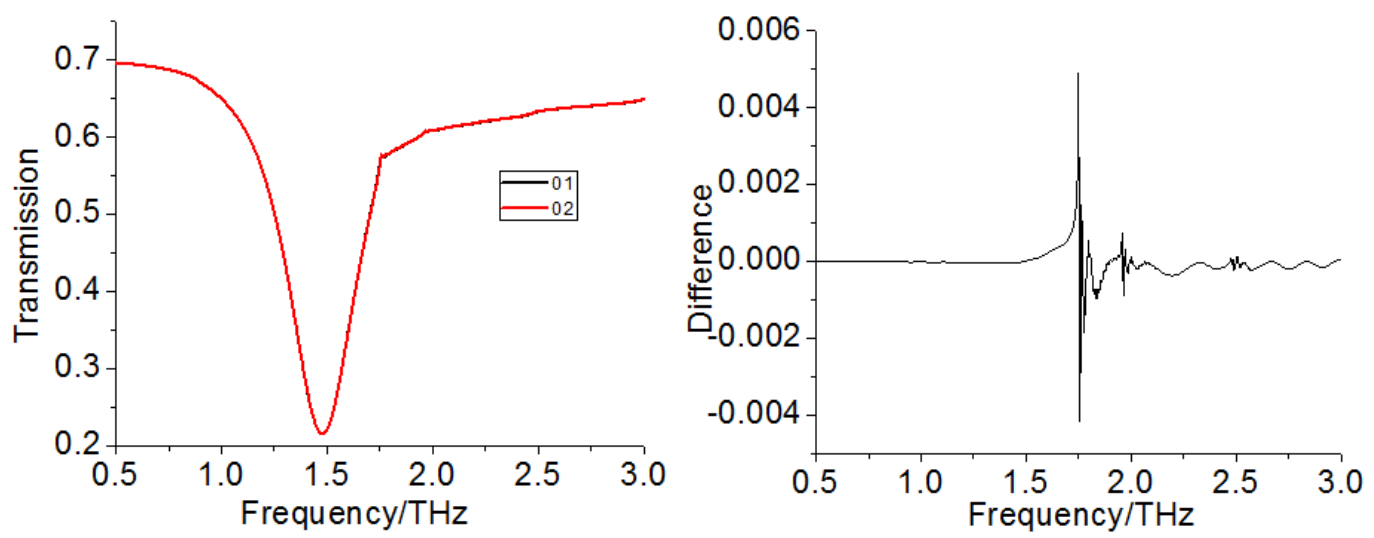

Figure 4.22. Transmission curves of Bowtie antenna 1 for two cases (reducing period versus adding more antennas for periodic boundary case) and their difference
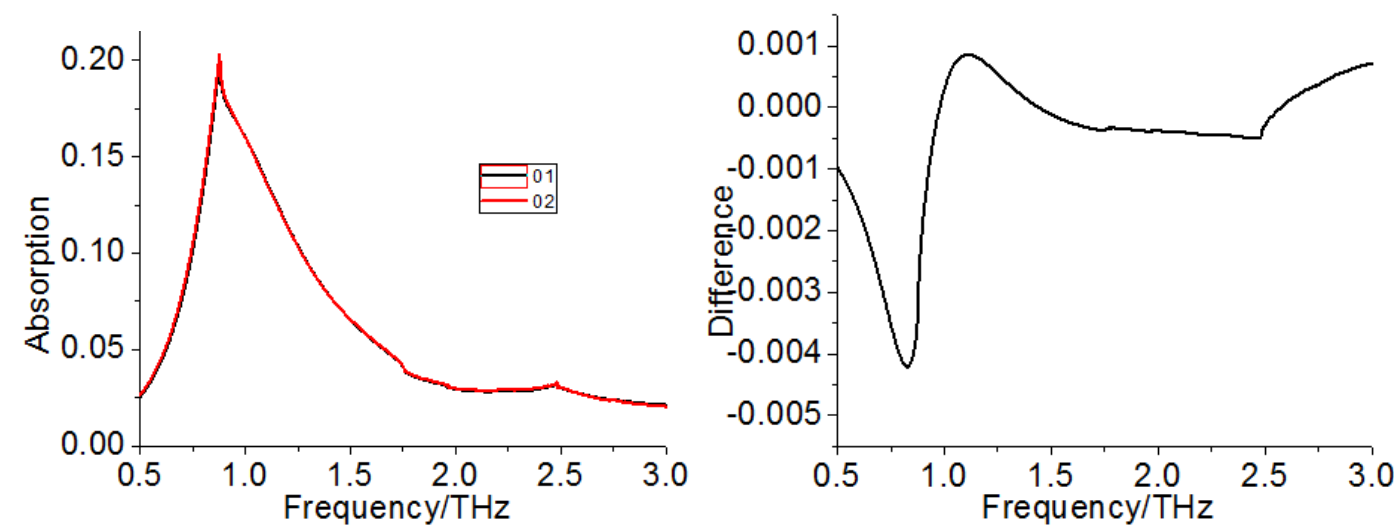

Figure 4.23. Absorption curves of Bowtie antenna 1 for two cases (reducing period versus adding more antennas for periodic boundary case) and their difference

After observing Figure 4.22 and 4.23, the conclusion is that the curves almost overlap with each other and the difference is negligible. Reducing the period is equivalent to the case in which one fills more antennas in one unit (the simulation region). Therefore, we could simply change the size of the unit area and only include one antenna in the region.

Another similar test was performed. Since the boundary condition around the simulation area is periodic, modeling of one antenna is equivalent to a 2-D infinite antenna array. In order to verify this feature, three simulations are implemented: they have $1 * 1,2 * 2,3 * 3$ antennas/pixels within the boundary respectively. The unit antenna is the same one used 
for the above polarization comparison. Theoretically, the responses of these three should be equal to each other. The modeling result is shown below. The three curves almost overlap together, indicating that our simulations make sense in terms of the boundary condition utilized. There is little numerical artifact.

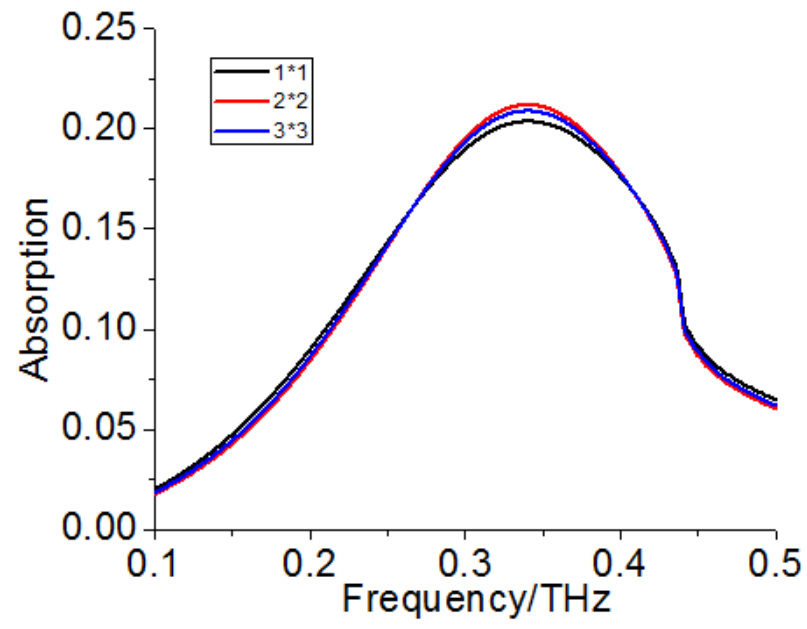

Figure 4.24. Absorption results for simulations checking the effect of periodic boundary. Three simulations have $1 * 1,2 * 2,3 * 3$ antennas/pixels within the boundary respectively.

\subsection{Load resistance}

A simple exploration of the effect of resistance on the absorption is implemented here. The conductivity of the resistor material in the model could be adjusted to control the resistance. The variation is from 20 to $100 \mathrm{ohms}$ as indicated in Figure 4.25.

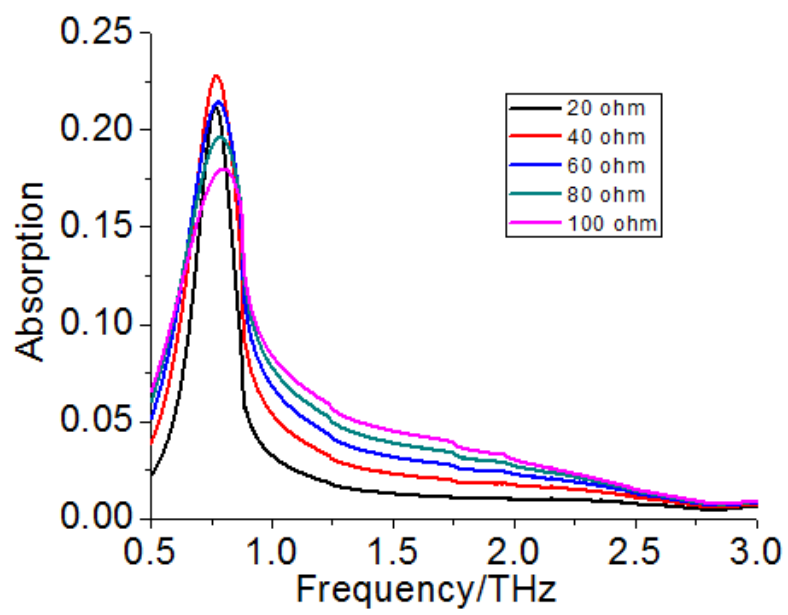

Figure 4.25. Absorption curves for Bowtie antenna 3 (except that the length is changed to be 40 microns for one element) for different resistances 
The most obvious observation is that the resonant location of the system does not change when the resistance varies. It is noted that the peak value does not monotonously increase. It is not the case that larger resistance would ensure more absorption of energy. This fact may be related to the impedance mismatch between the antenna and the detector [55]. When this impedance is matched, the energy conversion efficiency would be higher.

\subsection{E-field distribution from antenna to detector}

In this part, three simulation monitors are set to collect E-field distribution across the metal contact. This would help understand how the antenna works.

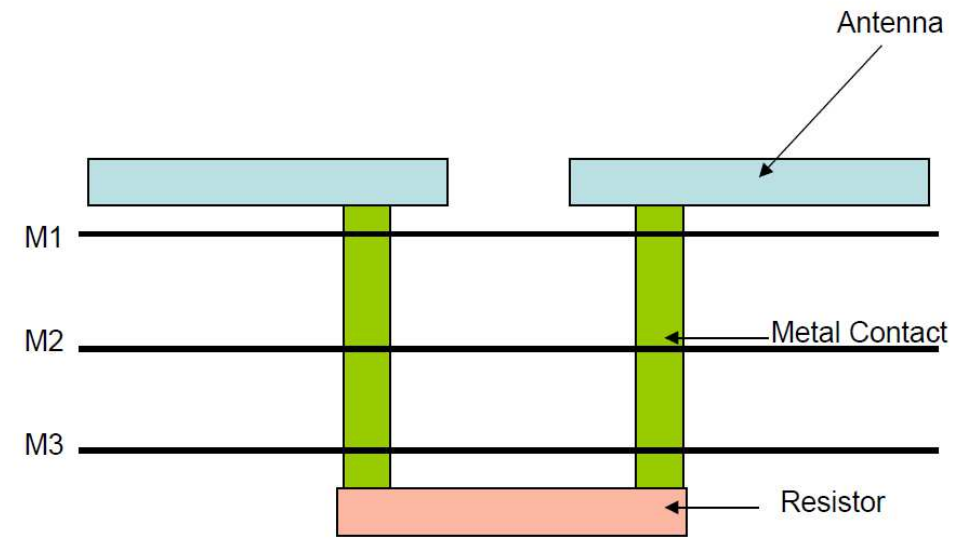

Figure 4.26. Side profile of antenna with the locations of the three monitors indicated. Three monitors (M1, M2, M3) are put across the metal contact to detect the E-field distribution information. Not to scale.

Parameters of the bowtie antenna used:

Inner width: 1 micron

Outer width: 3.1 microns

Length of each element: 18.6 microns

Gap: 2 microns

Unit size/period: $40 * 40$ square microns 
The 2D E-field distributions (for 1.52THz) are shown in Figure 4.27-4.29 for the three monitors:

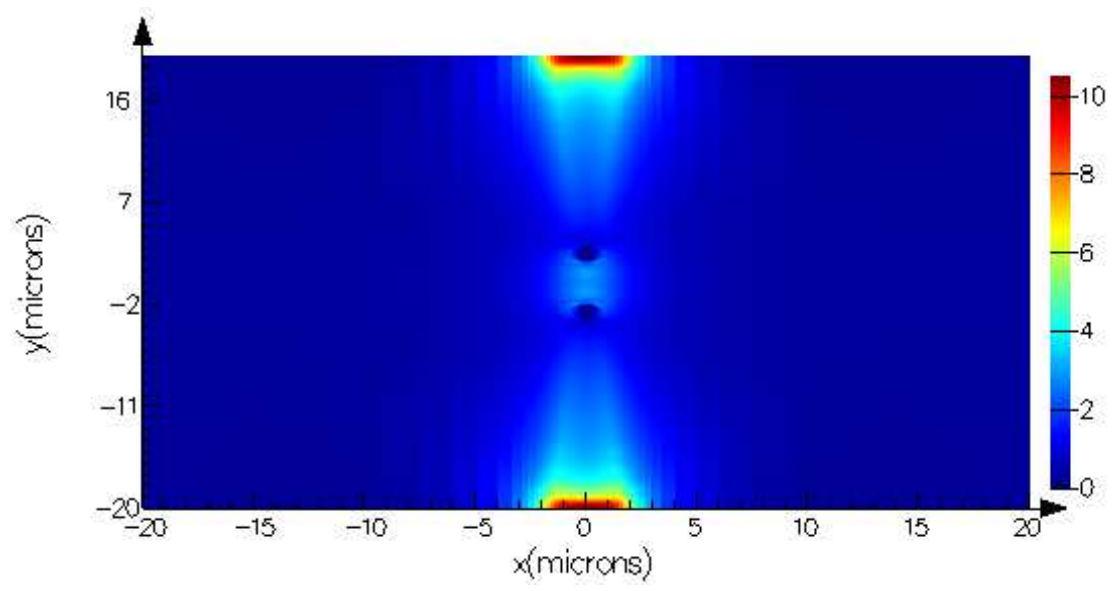

Figure 4.27. M1 E-field distribution. Unit of E field is $\mathrm{V} / \mathrm{m}$.

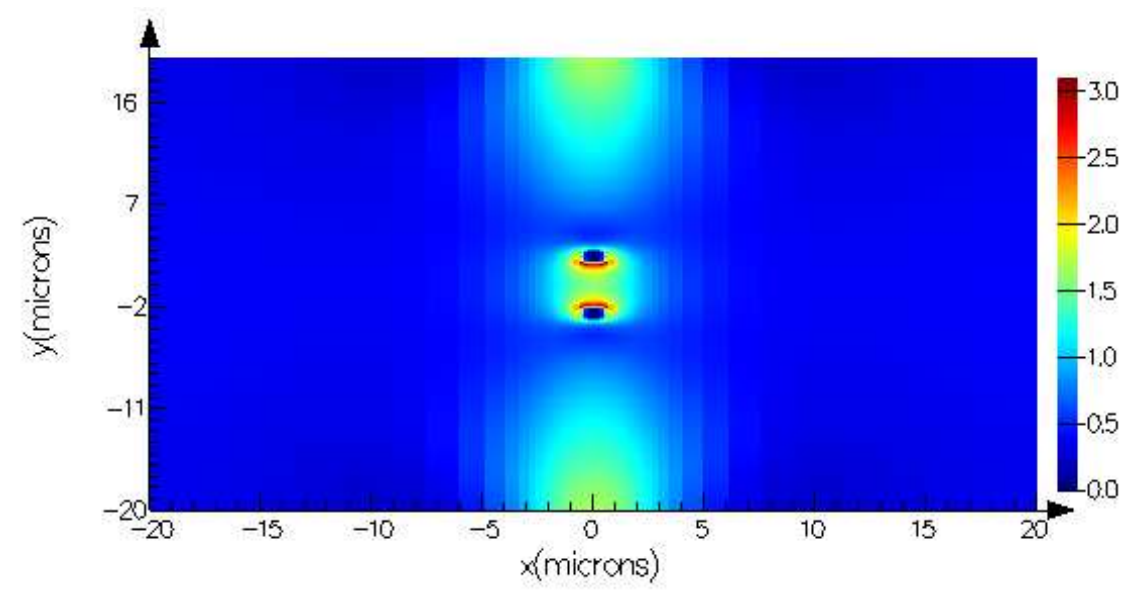

Figure 4.28. M2 E-field distribution. Unit of E field is $\mathrm{V} / \mathrm{m}$.

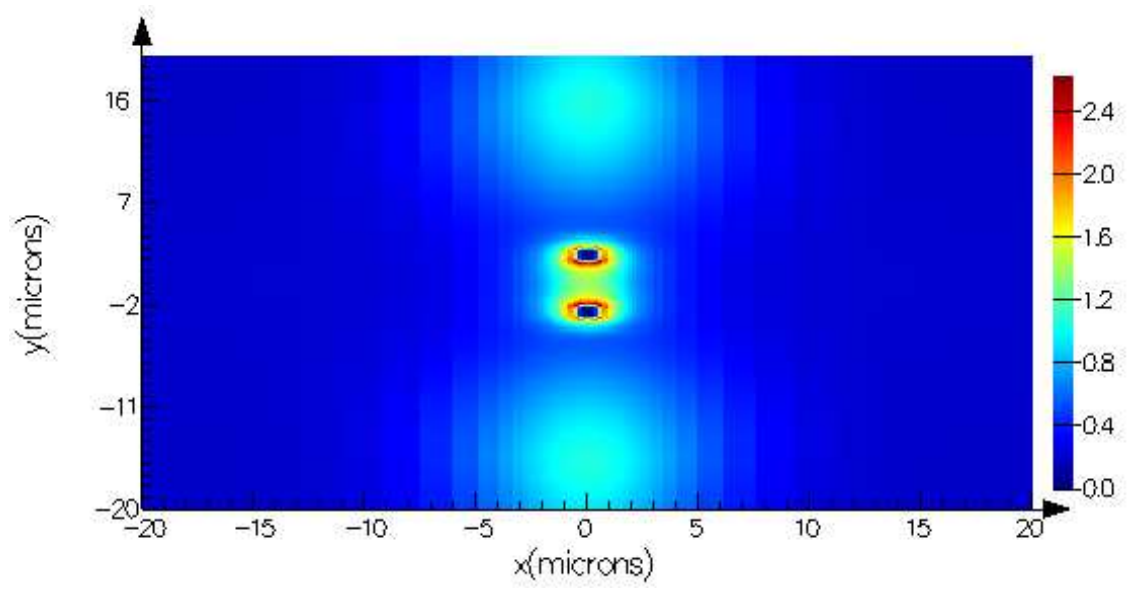

Figure 4.29. M3 E-field distribution. Unit of E field is V/m. 
For M1, which is close to the antenna, E field concentrates at the two ends. However, as monitor moves downside, the field tends to focus toward the middle part. This simulation verifies that the antenna qualitatively works as expected. The antenna of relatively larger shape functions as a collector that draws incident light that impinging on a large area to the central absorbing element, greatly enhancing the energy efficiency and detection sensitivity.

\subsection{Antenna thickness}

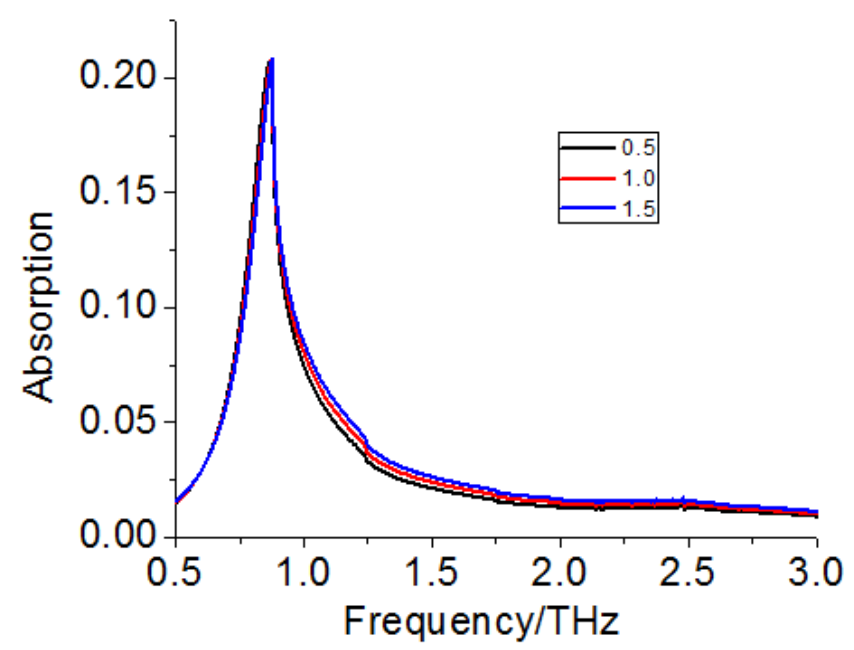

Figure 4.30. Absorption curves for Bowtie antenna 1 (pixel pitch is $100 * 100$ square microns) of different aluminum thicknesses from 0.5 to 1.5 microns

Figure 4.30 indicates that within the range from 0.5 to 1.5 microns, the thickness of the aluminum antenna could hardly affect the frequency response. Therefore, thicker antenna should be defined in the structure, allowing for larger grid in order to reduce computation time. At what thickness scale an effect will be seen requires further simulations. 


\section{215GHz \& 188GHz Antenna Design}

Now that we obtain some knowledge through various tests in the last chapter, we have the ability to design a specific antenna for the desired band. In this chapter, the main focus is to design a broadband terahertz antenna at $215 \mathrm{G} \mathrm{Hz}$. Some practical issues will be addressed as the designing process goes on. Moreover, one transmission experiment design utilizing an available $188 \mathrm{GHz}$ source will be implemented in order to verify the validity of the FDTD simulations. Finally, the angular antenna pattern will be considered for further applications like antenna gain calculation.

\section{1. $215 \mathrm{GHz}$ bowtie antenna design}

\subsubsection{Frequency design}

For the three bowtie antennas of the first generation, the resonant frequency information is listed in Table 5.1 .

\begin{tabular}{|c|c|c|c|}
\hline & Bowtie 1 & Bowtie 2 & Bowtie 3 \\
\hline Arm length/micron & 33.2 & 18.8 & 47.6 \\
\hline Antenna length/micron & 68.4 & 39.6 & 97.2 \\
\hline Peak absorption frequency/THz & 0.875 & 1.625 & 0.625 \\
\hline Peak absorption wavelength/micron & 343 & 185 & 480 \\
\hline
\end{tabular}

Table 5.1. Resonant frequency information for the three bowtie antennas of the first generation. The first row is length for one arm of the antenna. Since the gap between the two arms is 2 microns, the antenna length (the second row) is two times the values in the first row and then plus the gap. 


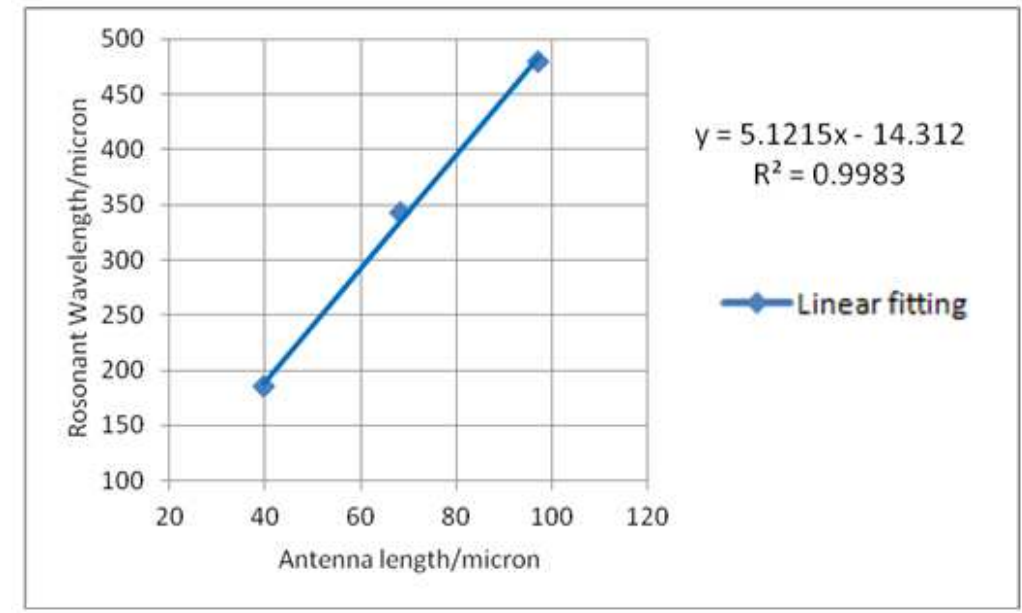

Figure 5.1. Linear equation fitting between the antenna length and resonant wavelength for first generation bowtie antennas

The linear equation derived from the above data is

$$
\mathrm{Y}=5.1215 \mathrm{X}-14.312
$$

where $\mathrm{X}$ is the total length of the antenna in microns and $\mathrm{Y}$ is the corresponding resonant wavelength. This could be utilized to design the $215 \mathrm{GHz}$ antenna. The three data points could arrive at a relationship that is very close to linear. The wavelength at $215 \mathrm{GHz}$ is equal to 1395 microns. Therefore, according to the above equation, the estimated antenna length should be 275 microns. Then, from here, we shall simulate antenna whose length is around this numerical value for $215 \mathrm{GHz}$ detection.

Now that we have an estimated length ( 275 microns) of the bowtie antenna for $215 \mathrm{GHz}$, 6 initial designs around that length are simulated here. Definitions of the antenna parameters are illustrated in Table 5.2 and Figure 5.2. For these six designs, the pixel pitch is $330 * 330$ square microns. 


\begin{tabular}{|c|c|c|c|}
\hline Antenna design \# & Length for one arm/micron & Gap/micron & Angle/degree \\
\hline 1 & 110 & 5 & 30 \\
\hline 2 & 130 & 5 & 30 \\
\hline 3 & 150 & 5 & 30 \\
\hline 4 & 130 & 10 & 30 \\
\hline 5 & 130 & 5 & 45 \\
\hline 6 & 130 & 5 & 60 \\
\hline
\end{tabular}

Table 5.2. Geometric parameters of the 6 antenna designs for the modeling

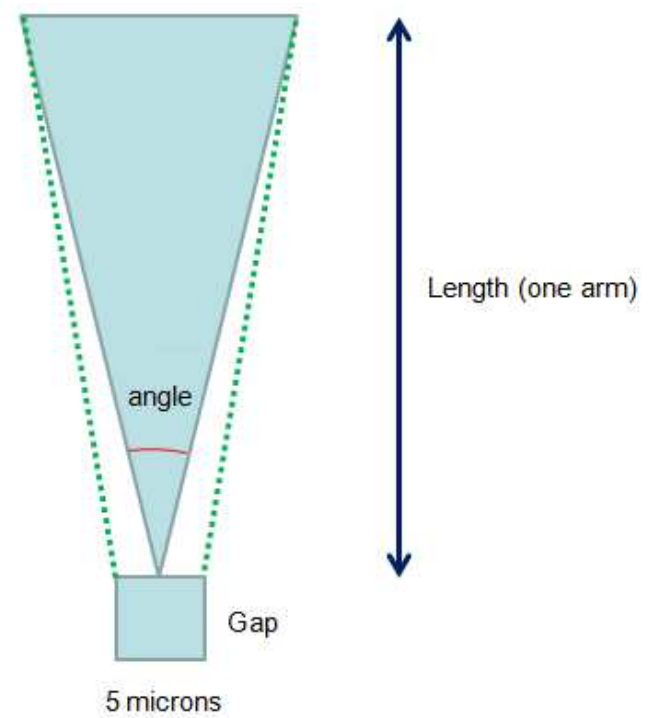

\section{One arm and central resistor of the antenna}

Figure 5.2. Schematic of one arm and central resistor for the 6 antenna designs. Not to scale.

Note the angle in the schematic, in order to make the arm well connected to the central resistor, an approximation is applied. The outer two end points of the arm could be determined by the length and the angle. After that, the two green dash lines are added to make the triangle transform to a trapezoid. Since the width of the resistor ( 5 microns) is very small compared to the length of one arm (110 microns minimum), this approximation has little effect. When the gap is changed, the conductivity of the resistor 
is altered to make the resistance fixed at $50 \mathrm{ohms}$. This resistance value is not optimal for maximum energy transfer so the current focus is to identify the resonant location of the antenna.

Observing the information table for the 6 designs, we have undertaken several studies to determine the effects of length, angle and gap. Now we will check these separately.

For the first subsection, antenna 1,2,3 have different lengths.

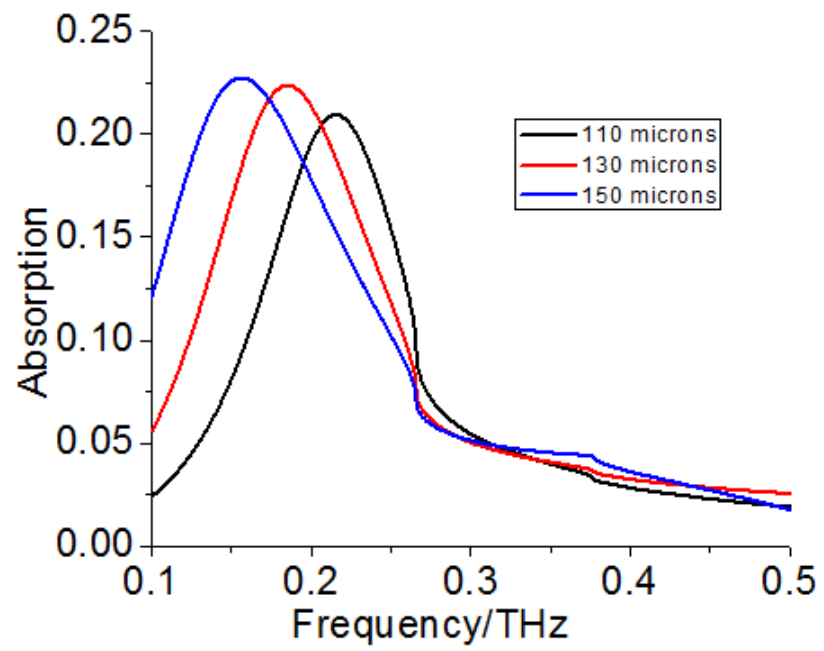

Figure 5.3. Absorption responses for antennas of different lengths

These three antennas have same angle and gap. A longer antenna should have a larger resonant wavelength, which corresponds to a smaller frequency. Therefore, from the 110 to 150 microns, the location of peak absorption shifts to left. This is an obvious feather in Figure 5.3. The resonant frequencies are $215 \mathrm{GHz}, 185 \mathrm{GHz}$ and $156 \mathrm{GHz}$. Also, a longer antenna would cover a larger area, making the longer antenna has a slightly uprising curve, absorbing a larger portion of the incident radiation.

In the second subsection, antenna 2, 5, 6 have the same length, gap and different angles. 


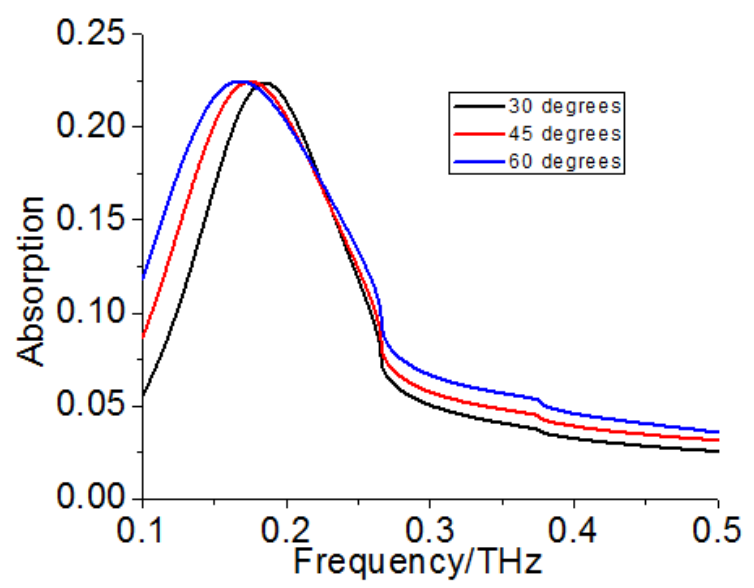

Figure 5.4. Absorption responses for antennas of different angles

In this case, antennas have the same length. However, a larger angle makes the antenna behaves as if it is a little longer than the thinner ones. So the 60-degree antenna has the smallest resonant frequency. The difference between these three curves is smaller compared to the length effect shown in the last part.

Now, we turn to briefly look at the effect of gap. Between antenna 2 and 4, the only difference is the gap seen from Table 5.2. However, since the total length of the antenna equals to two times the arm length and plus the gap. The difference between two curves in Figure 5.5 is not negligible, but this may not be the cause of the gap. Analysis for the effect of the gap will be presented later in this paper.

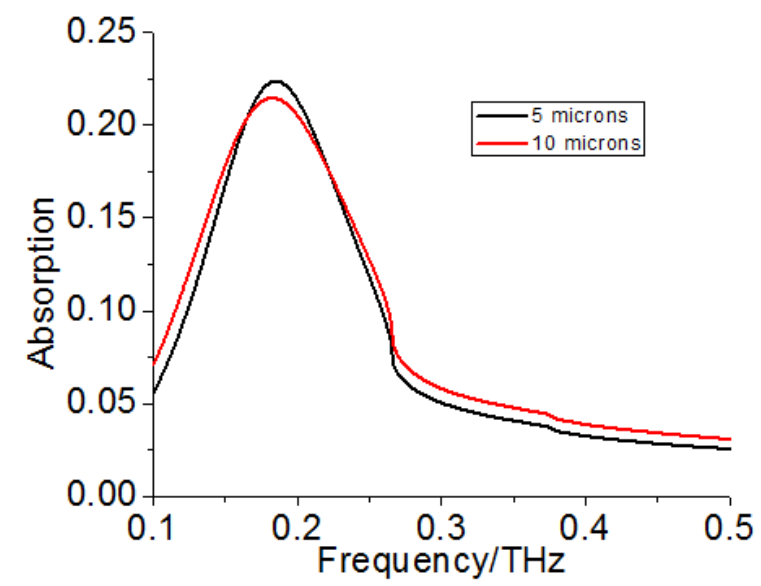

Figure 5.5. Absorption responses for antennas of different gaps 
In section 4.1 where the material parameters were discussed, we had two options for the silicon oxide model. Here, in Figure 5.6, we show the simulation results of these two schemes indicating that there is little difference. Firstly, we have experimental data within Lumerical and could then extend those data to our desired frequency range by multi-coefficient fitting. Secondly, the index values are extracted from the design plot (Figure 3.9), real and imaginary parts of the index are 2.05 and 0.0002 respectively (calculated from the data indicated in Figure 3.9, real permittivity and loss tangent are 4.2 and 0.0002 . [44]).

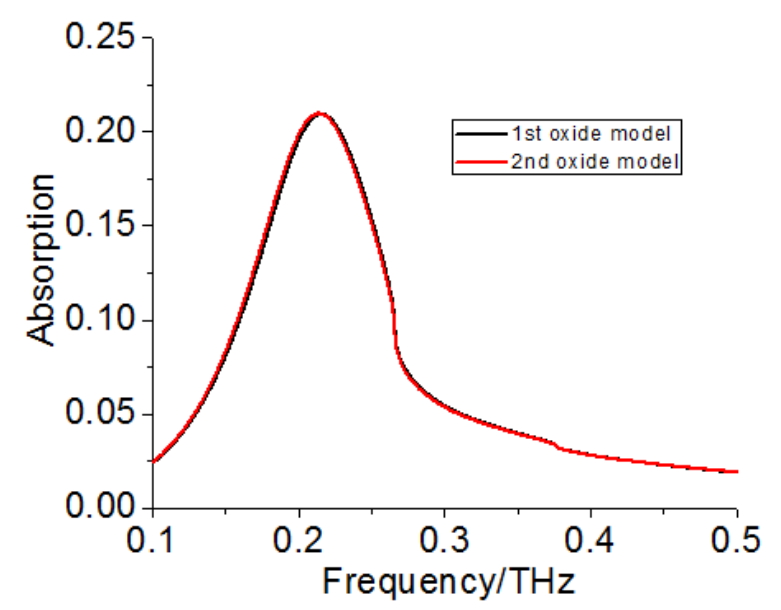

Figure 5.6. Absorption response comparison between two different oxide models

Here, we show the comparison results between these two cases that there is little difference. Therefore, we could use either of two as the oxide model from now on.

For now, in the simulations, the antenna is on a 2-micron thick oxide layer and silicon substrate. However, in reality, the antenna may be surrounded by the oxide, also there may exist a silicon nitride layer on top of that (as shown in Figure 3.9). So we calculate the absorption for the three conditions indicated in Figure 5.7. The design is based on the first of those six (Table 5.2) except that the angle is changed to 60 degrees and the pixel pitch is extended to 350 microns. The thicknesses of antenna and nitride layer are 1 and 2 microns respectively. For nitride, the real and imaginary parts of the permittivity over the range of $0.4-2 \mathrm{THz}$ are 7.6 and 0.04 , corresponding to $\mathrm{n}=2.646+\mathrm{i} 0.0066$. 


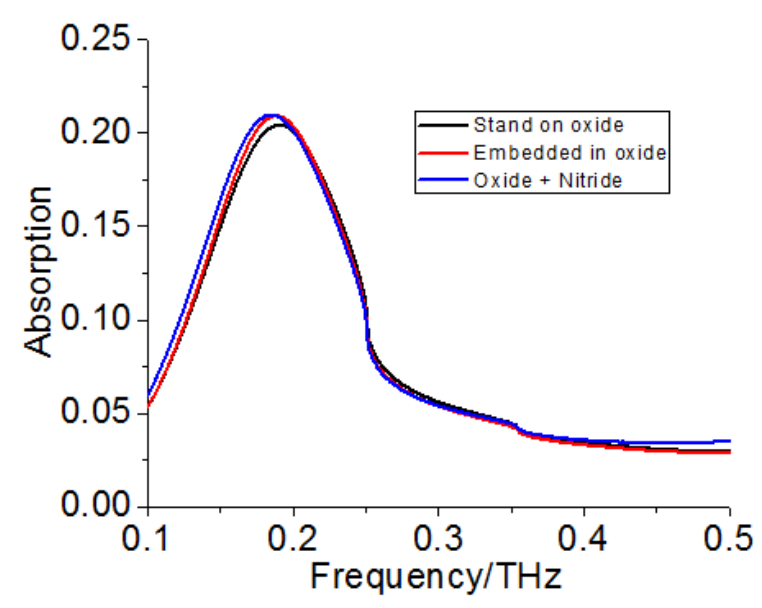

Figure 5.7. Absorption response comparison considering oxide and nitride configuration

Since the three response curves are pretty close to each other, we might use the simplest structure (antenna on oxide) to represent the actual configuration.

Previously, when we had the initial six test designs for $215 \mathrm{GHz}$, the definitions of the length/angle were a little confusing and were not very convenient to use. Therefore, it is better to alter the definitions a little bit as Figure 5.8 indicates.

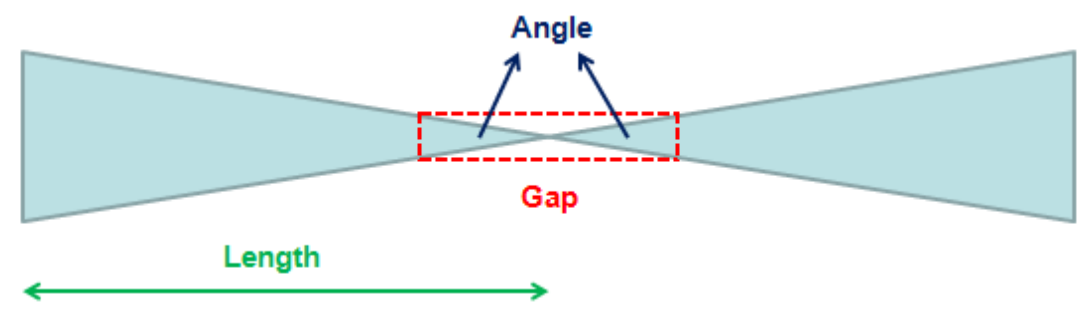

Figure 5.8. New definitions for the antenna gap and angle (not to scale)

In the new definitions, we first assume that the two triangular arms touch by their tips. The angle is inner angle of two corners of triangles that touch each other. After this, when the gap is known, a rectangle (red box in the figure) is formed. The width of the box is determined by the gap (how wide is the antenna at the gap ends). The antenna portion within this red box is etched to form the gap. Then the gap is filled with the resistor or the underneath load is connected to two arms via metal contacts. 
The advantage of these definitions is that no matter how long the gap is, the angle remains fixed. The length is measured from the center to one end, so the total length is just two times the length.

The absorption curve of a bowtie antenna (with length $=110$ microns, angle $=30$ degrees and gap=10 microns) for this new definition could be seen here. Compared to the first antenna in Table 5.2, this curve still centers at $215 \mathrm{GHz}$ and will be utilized as a reference for the subsequent analysis.

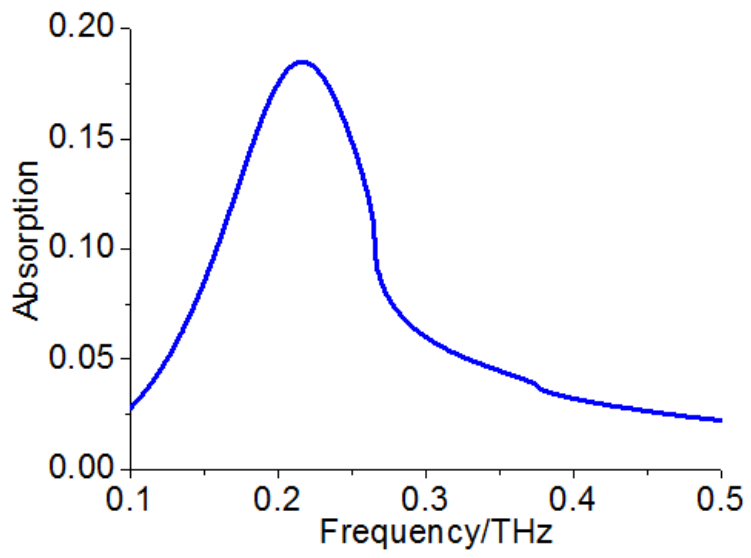

Figure 5.9. Absorption for a new defined bowtie antenna with length=110 microns, angle $=30$ degrees and gap $=10$ microns

\subsubsection{Effect of slots in the antenna}

Now, there exists an issue with the manufacturing. The design rule check (DRC) restricts that we can not make continuous aluminum across the whole antenna. As a solution, some etches through the antenna could be able to break the continuity. Several tests were made to check how the etched slots would affect the frequency response of the antenna. 

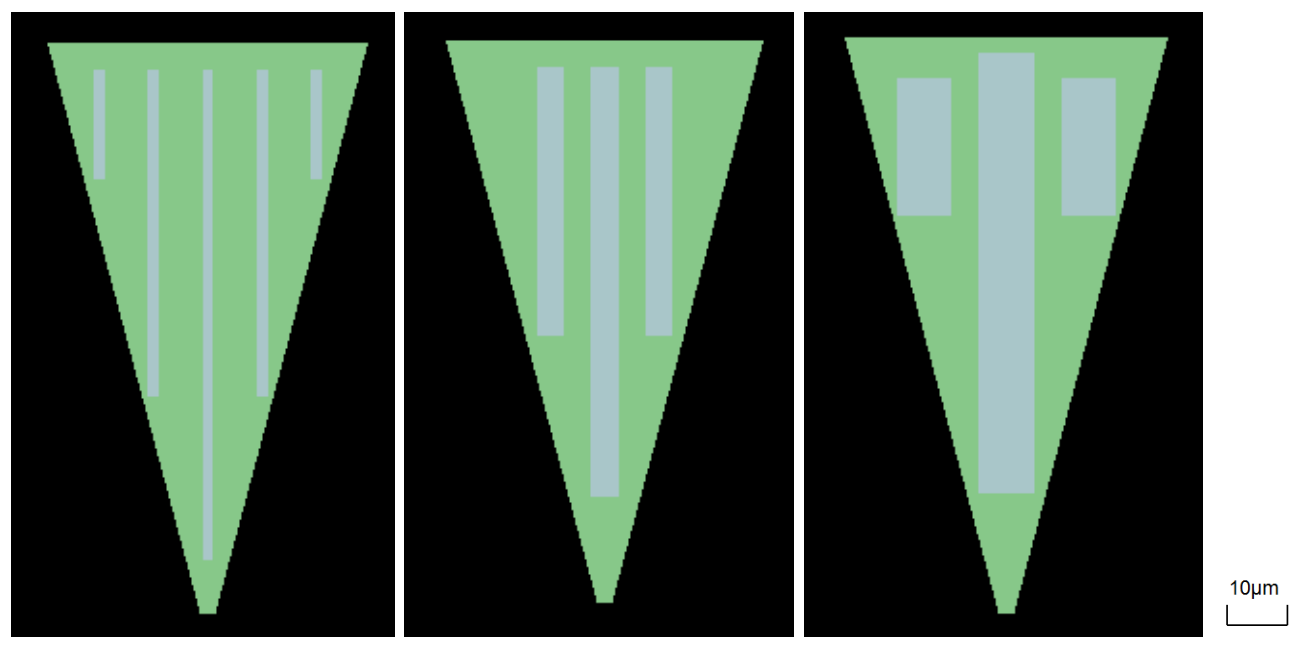

Figure 5.10. Test structures to check the slot effect. Here, only one arm of the antenna is shown, where the etched slots are of several rectangles inside the arm. The widths of the etched slots are 2, 5 and 10 microns respectively.

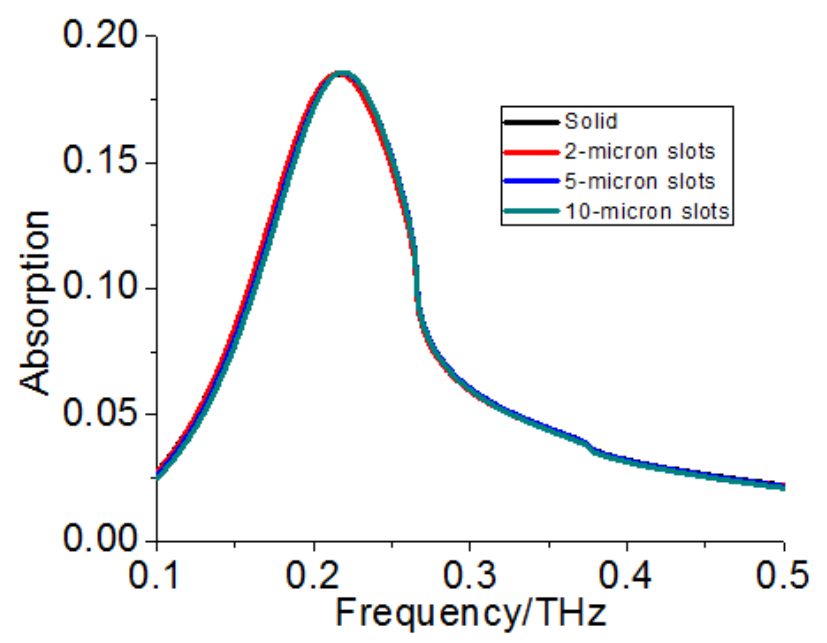

Figure 5.11. Absorption simulations for antennas with test slots

For the three test structures, the widths of the etched slots are 2, 5 and 10 microns respectively. The meshes are fine enough for the algorithm to 'see' the slots (the mesh sizes are $0.5 * 1,1 * 2,1 * 2$ square microns). For the last two, since they have slots of larger width, the number of slots is less than that of the first one. The solid antenna is the same one as used in Figure 5.9. The absorption curves for slot antennas almost overlap together with that of the solid antenna, demonstrating that the slots in the three test designs have no effect on the absorption response. 


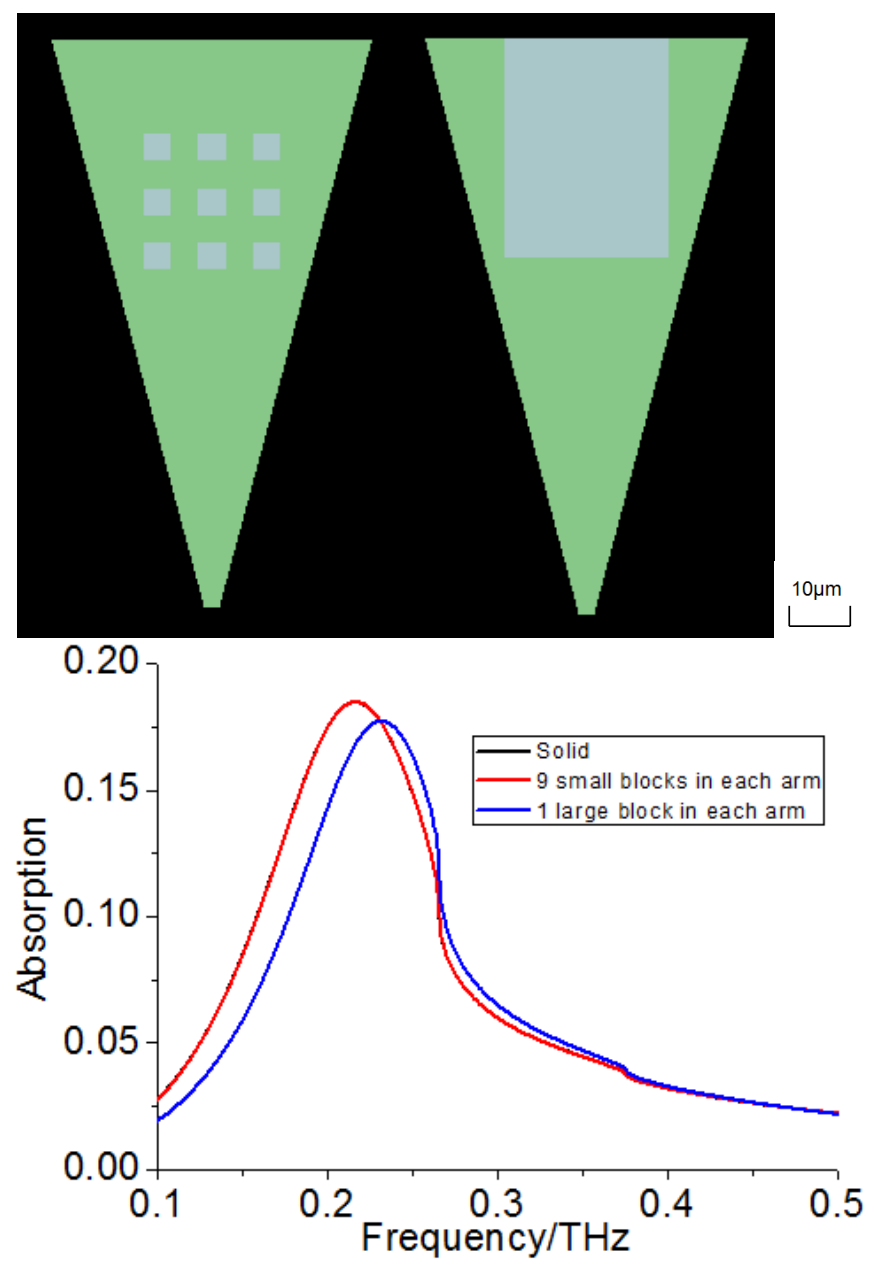

Figure 5.12. Two tests for antennas with etched blocks. The sizes of the blocks are $5 * 5,30 * 40$ square microns respectively.

Two more tests were made. For the first, there were nine small etched blocks in each arm. For the second, there was one large etched block in each arm. The sizes of the blocks were $5 * 5,30 * 40$ square microns respectively. As Figure 5.12 is showing the comparison between the solid antenna and the antenna with etched blocks, the antenna with small blocks has the same response with the solid one. However, for the antenna with a large block in each arm, the absorption curve drops down a little and shifts to right. In conclusion, when the etched slots and blocks are small compared to the wavelength of the radiation (hundreds of microns), they have little effect on the absorption response of the antenna. The system is still able to couple the incident light that falls on the slot/block portion. When the blocks become large 
enough, it could affect the frequency response. Here the dimension of the etched slot is about $1 / 50$ of the wavelength, a general statement of when the slot is large enough to affect the frequency response requires further exploration.
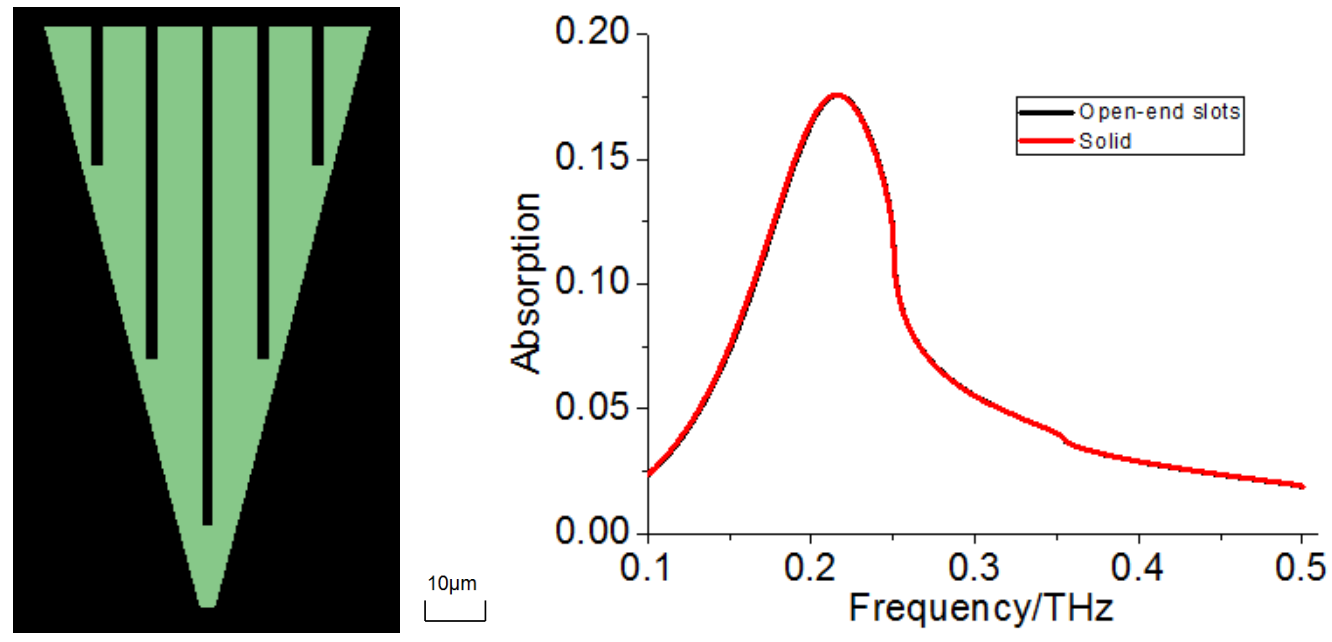

Figure 5.13. The antenna design with open-end slots and its absorption response.

The width of the slots is 2 microns

Next, the test is about the design with open-end slots like the one shown in Figure 5.13. The length of one arm is 110 microns and the angle is 30 degree. The pixel pitch is $350 * 350$ square microns and the mesh size is $0.5 * 0.5$ square micron. The width of the slots is 2 microns. As expected, the solid antenna and the one with slots have the same absorption response, which is verified by Figure 5.13.

The design rule check tool in manufacture indicates that the aluminum could not be continuous in both two directions for 35 microns. Therefore, after testing in the design software, the following designs are valid to use. 


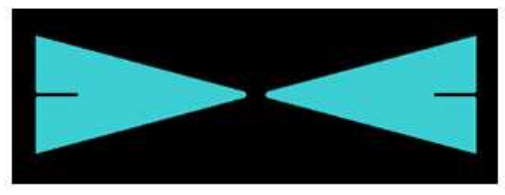

30-degree 110um antenna design with rounded corners
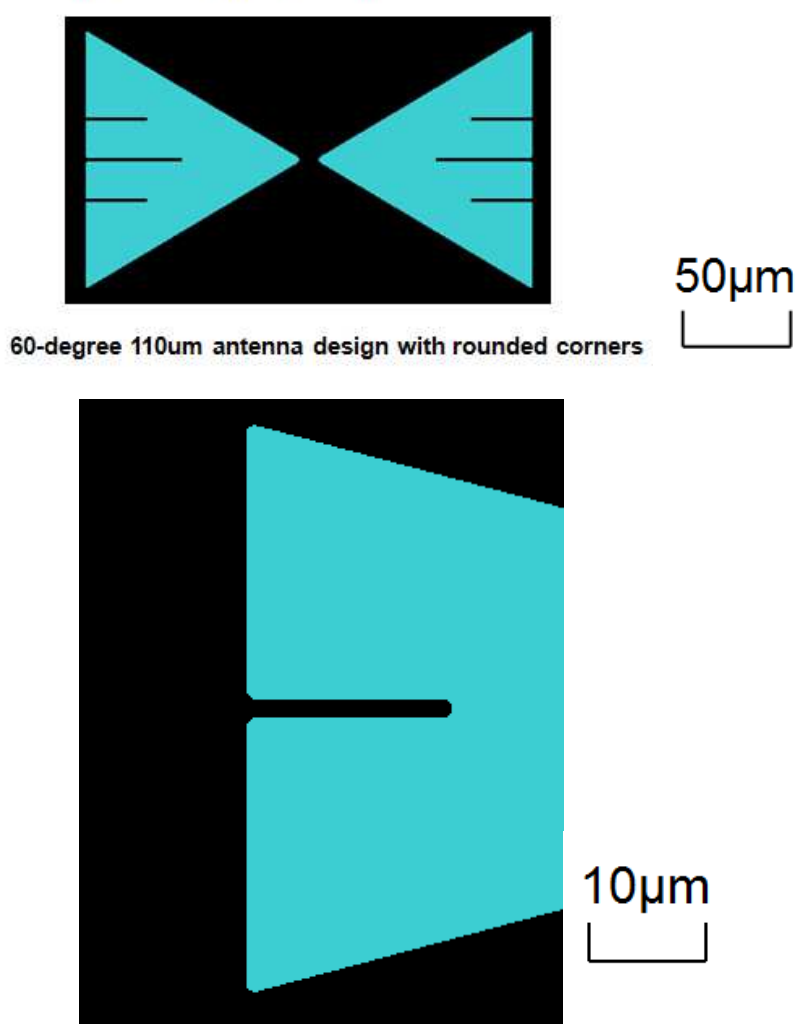

Figure 5.14. The designs that satisfy the manufacture rule and after corners are rounded

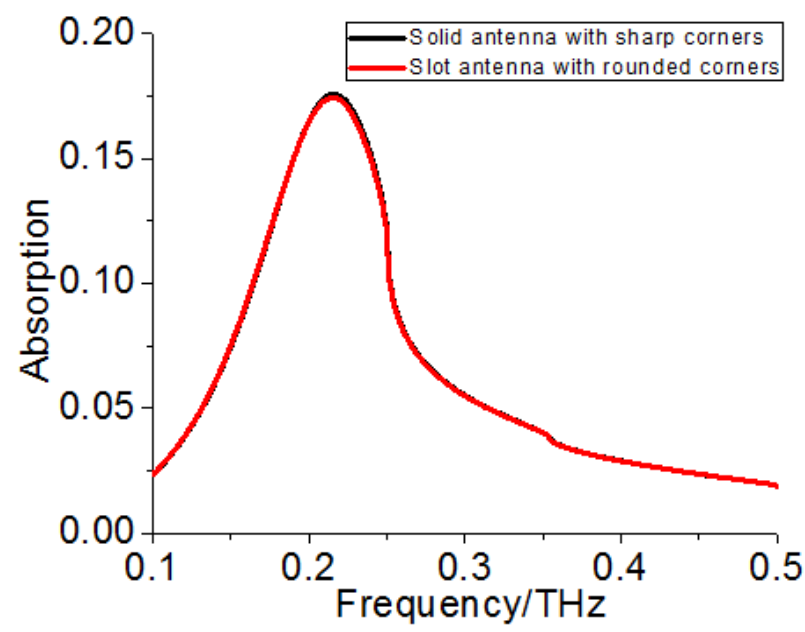

Figure 5.15. Comparison between solid antenna with sharp corners and slot antenna with rounded corners 
The comparison for absorption response between the solid antenna with sharp corners and the slot antenna with rounded corners is shown here. The size of the slot is around $2 * 20$ square microns; the dimension is small compared to the wavelength to make the response vary.

In order to help exploring the physics, the electric field distribution through the middle of the slot antenna (xy-plane) is calculated and displayed. It is noted that there is no resistor when modeling the e-field since the interest is in the field distribution for the two arms of the antenna without the underlying electronics. The mesh is $0.5 * 0.5$ square micron, which is fine enough to distinguish the slot and the bulk. We could check the E field distribution near the slot to verify that the simulation takes into account the effect of slots. Also, we could compare this distribution to some references, which will be seen in section 5.1.3.

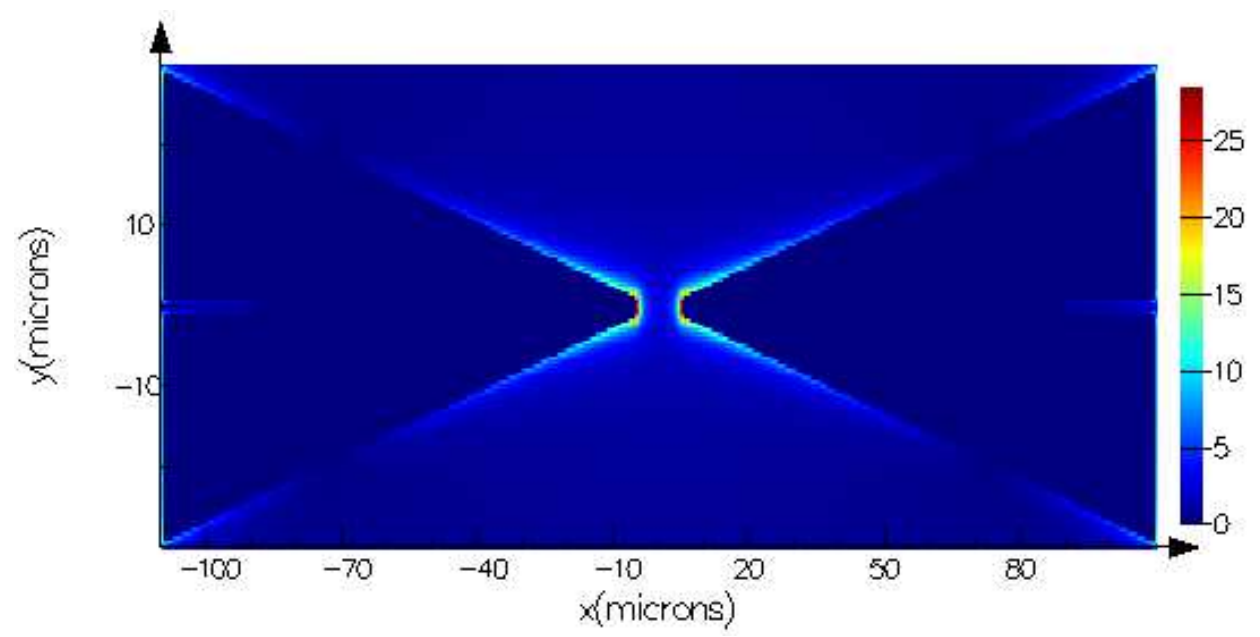

Figure 5.16. Electric field distribution @215GHz for the central plane of the slot antenna with rounded corners (the antenna is along the horizontal direction). Electrical field unit: $\mathrm{V} / \mathrm{m}$

Observing this profile, there is no field in the aluminum, which is certainly reasonable. Since the antenna material is set as perfect electrical conductor for the terahertz radiation, electric field can not penetrate into the metal, the field distributes along the edge of the antenna. The field focuses on the two tips of the antenna. In 
the slot areas, there is some field, indicating that the simulation does consider the effect of the slot, although there is no effect on the frequency response.
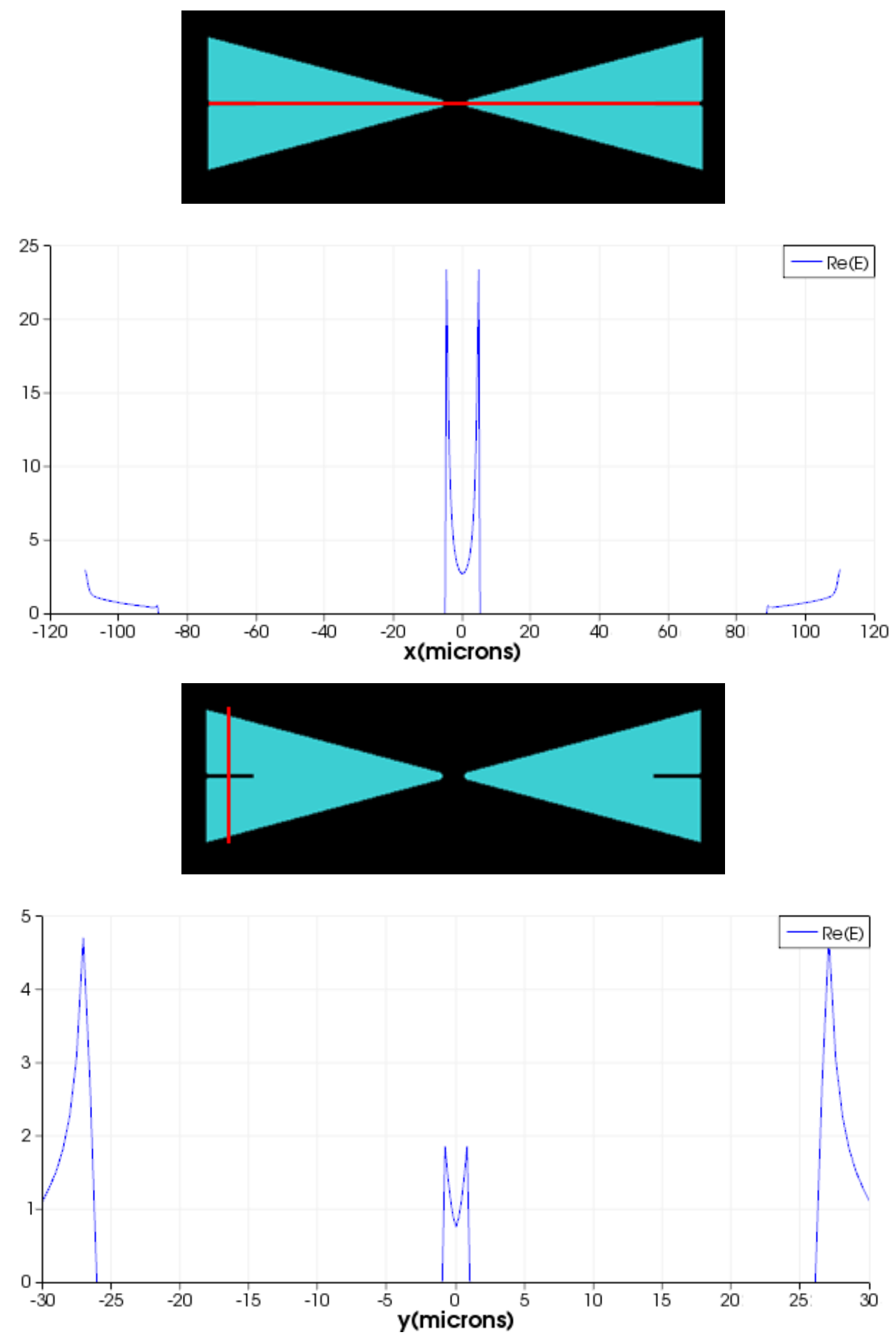

Figure 5.17. 1-D electric field distribution. Electrical field unit: V/m 
The 1-D field patterns demonstrate the electric field distribution well. For the first case of Figure 5.17, the red line is in the center of the antenna and along $\mathrm{x}$ axis. The values are extremely high in the central tips of the antenna and quickly drop down away from the edges. For the second red line, since the width of the open-end slot is very small, the field value in the center of the slot is not but close to zero.

\subsubsection{Effect of antenna gap}

If we decrease the gap and increase the inner width of the two arms, theoretically, we would see a much more uniform field across the gap. Figure 5.18 shows a test that has a gap of 5 microns and inner width from 5 to 40 microns. The mesh size is $1 * 1$ square micron. 

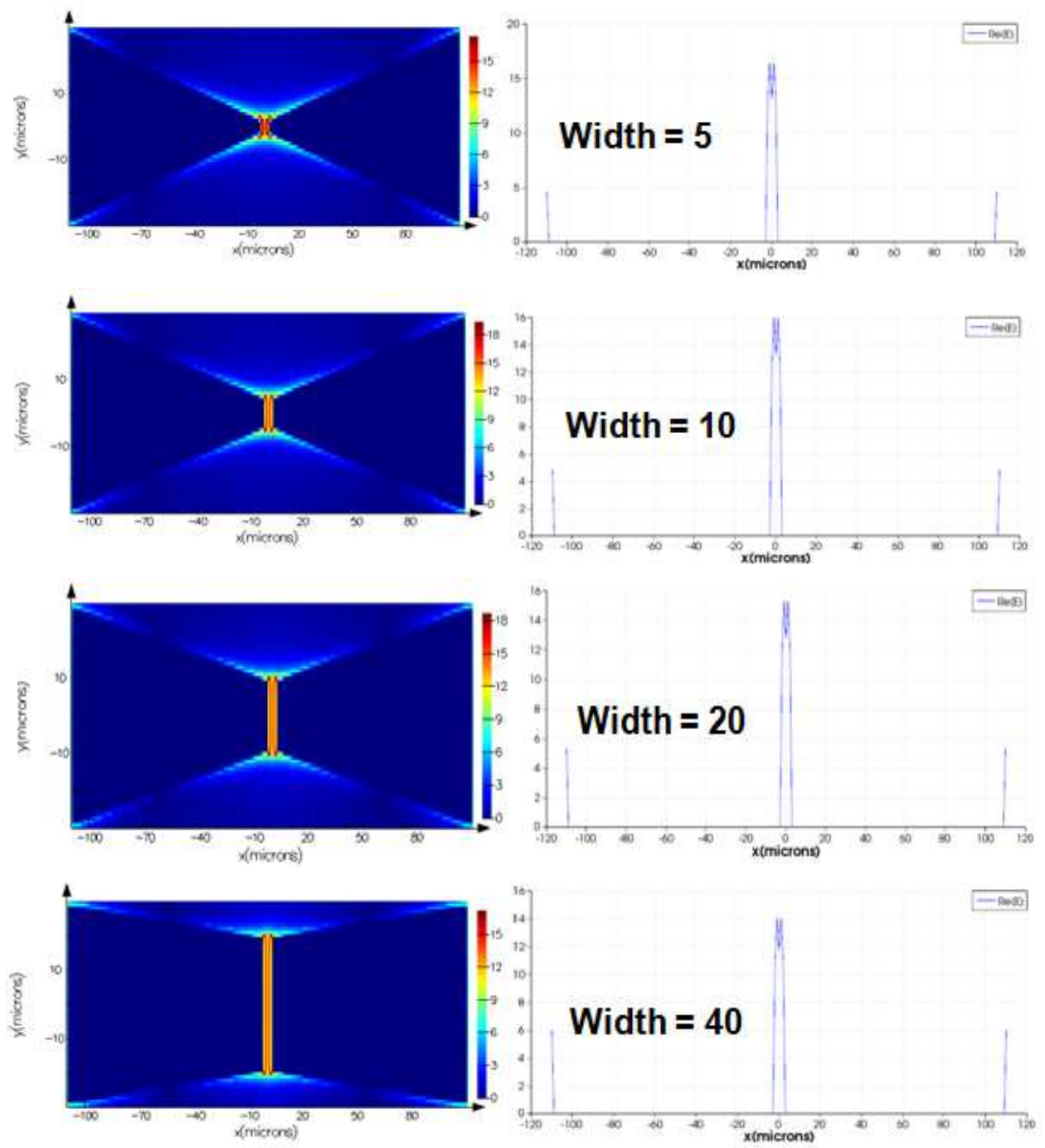

Figure 5.18. Field distribution for antennas with gap $=5$ microns and inner width from 5 to 40 microns. Electrical field unit: $\mathrm{V} / \mathrm{m}$
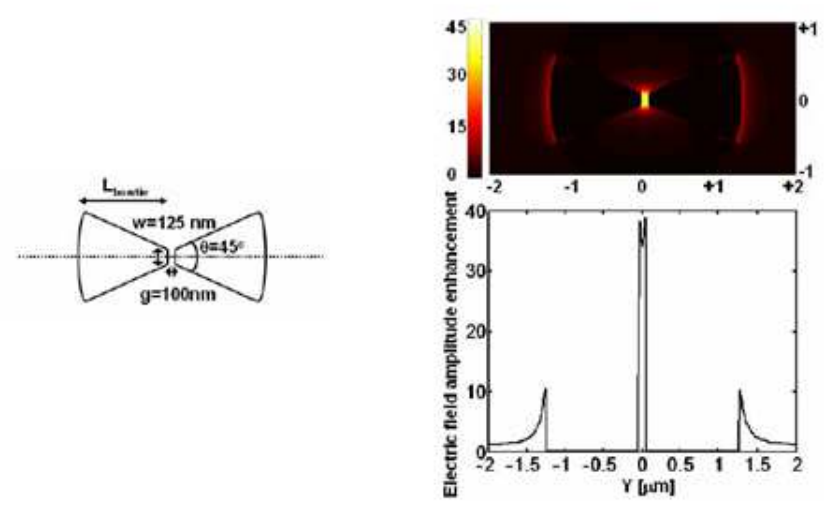

Figure 5.19. Electric field distribution from reference [56] 
Our field pattern in Figure 5.18 is similar to that of Figure 5.19. It is very intensive in the gap and the energy is along the edges. If the gap is small enough, it would almost be uniform across the two tips of arms. For our case, as the inner width increases, the peak value at the tip drops down a little. This may be explained by that the energy is distributed more widely for the wider arm.

Now, we'd like to see how the gap would affect the frequency response of the antenna. As in Figure 5.20, we tested three cases in which the gap is changed from 10 to 20 microns. We adjusted the conductivity of the resistor in the center to make the resistance fixed at $50 \mathrm{ohms}$. The results show that the length of the gap does not have a significant effect as long as the resistance keeps the same.

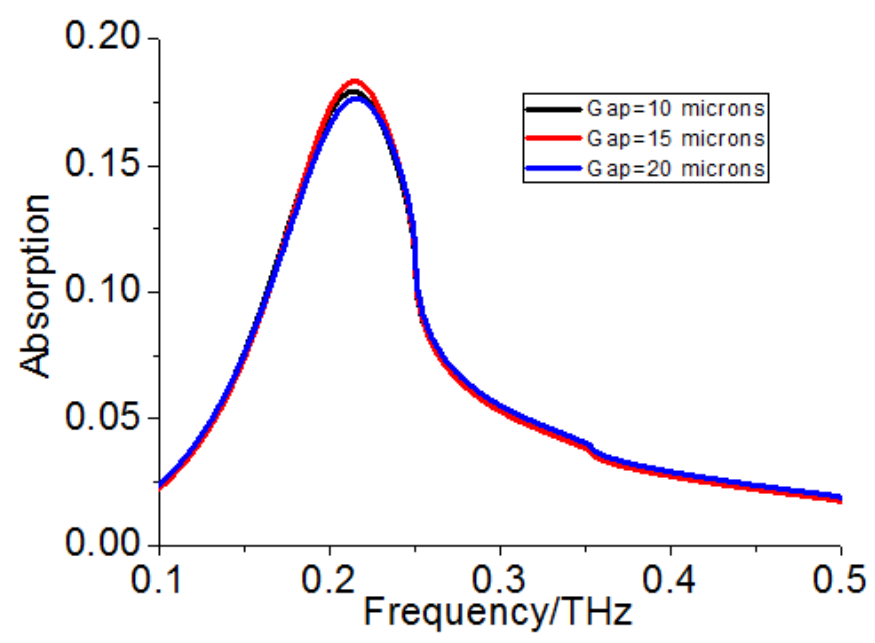

Figure 5.20. Absorption responses when the gap is changed from 10 to 20 microns 


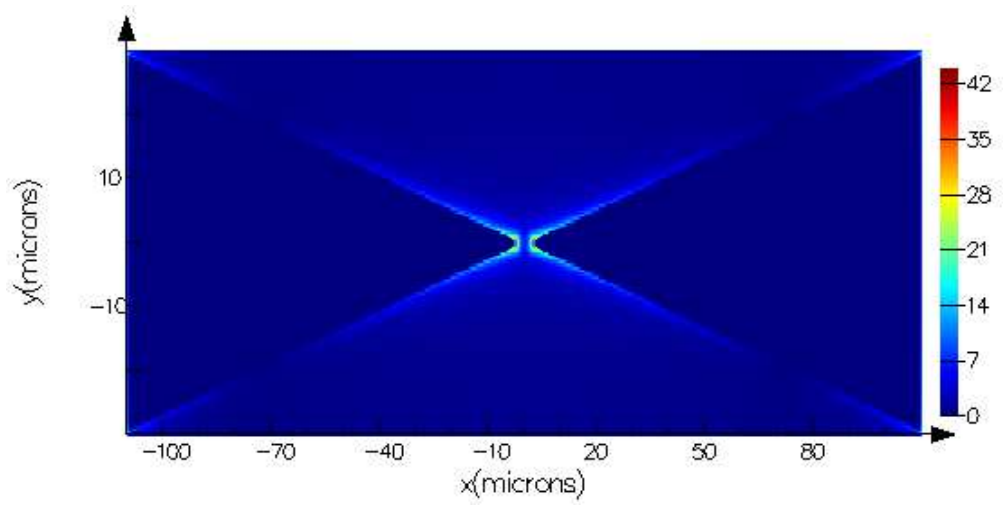

Gap $=5$ microns

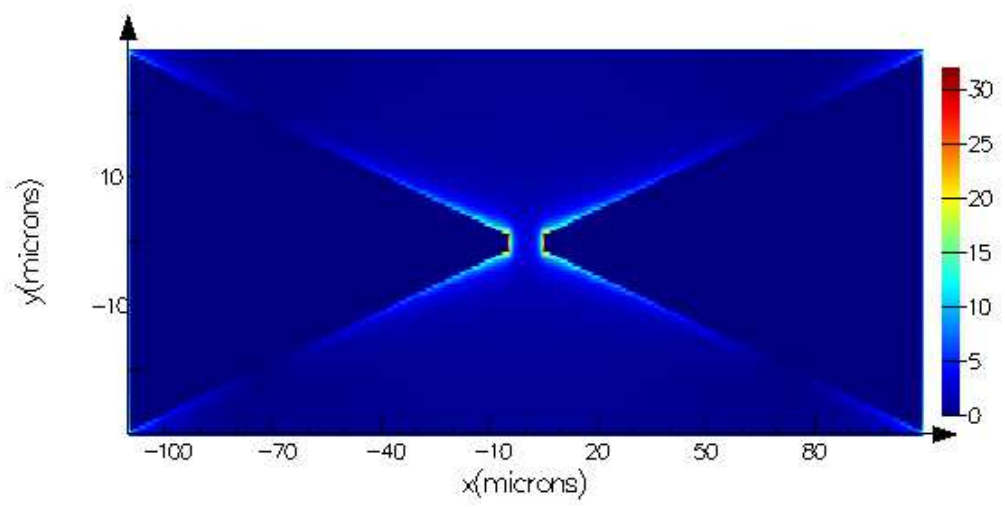

Gap=10 microns

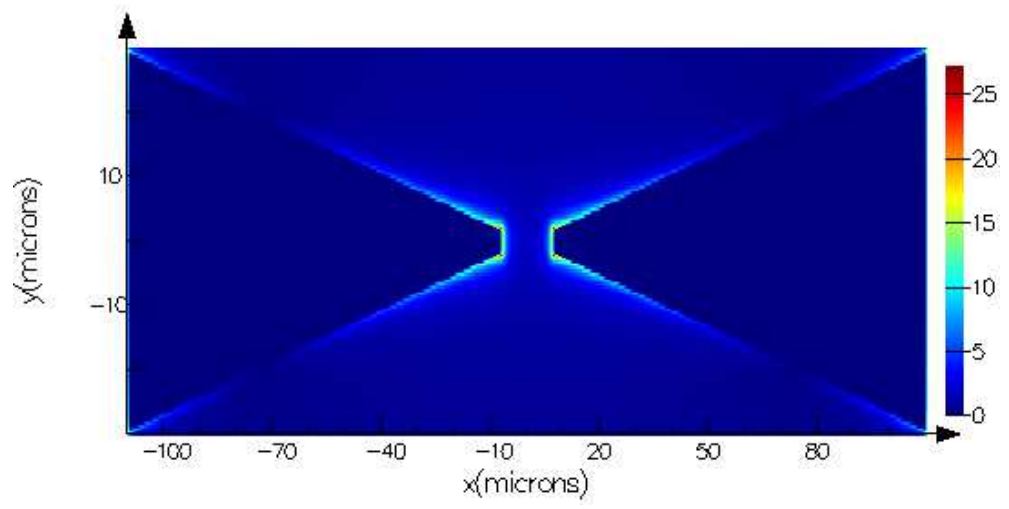

Gap $=15$ microns

Figure 5.21. Electric field distribution of the central plane through the antenna for variations of the gap between the two arms. Electrical field unit: V/m

However, variation of the gap could certainly affect the field pattern. From Figure 5.21 , as the gap increases, the peak field value decreases (When the gap is from 5 to 15 microns, the peak field values are around 42,30 and $25 \mathrm{~V} / \mathrm{m}$ respectively). 
Therefore, for applications which requires large near field like Raman Scattering, a small gap is the best choice.

Finally, we set the gap to be 2 microns (keep the inner and outer widths and change the arm length) and compare two different configurations. In the first one, the resistor is in the same plane and is directly connected to the two arms of the antenna; in the second one, the resistor is underneath the antenna and is connected to arms through metal contacts. The schematics are shown below:
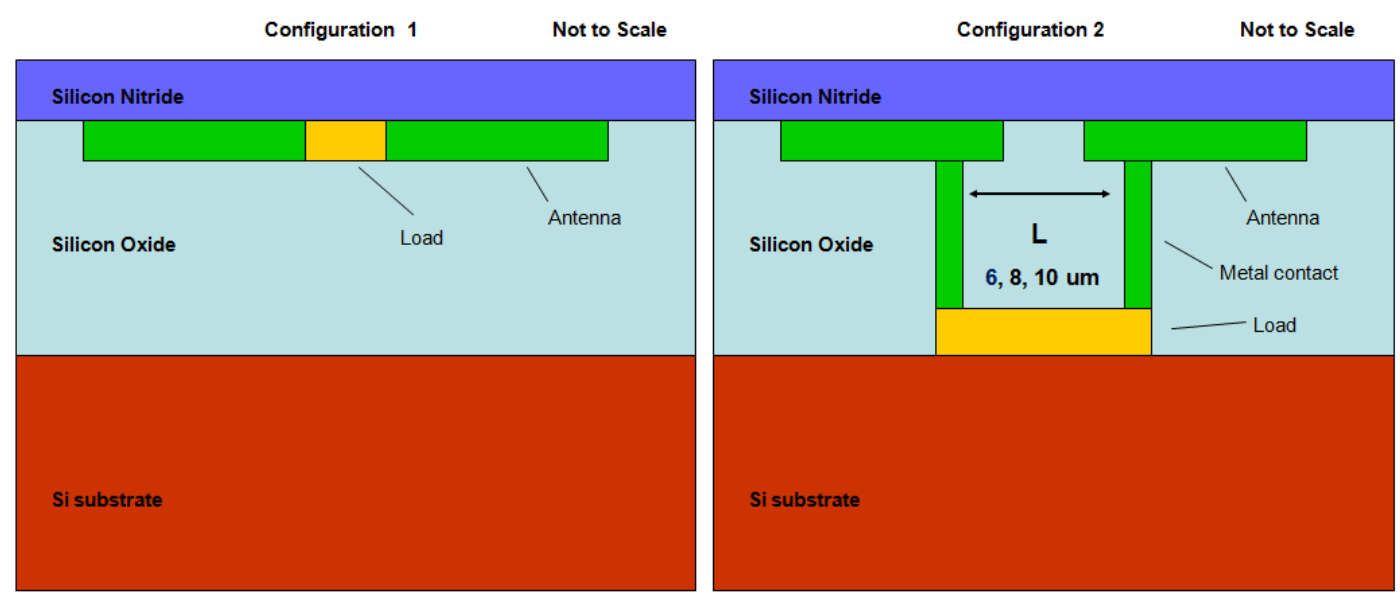

Figure 5.22. Two different configurations for the final antenna design with a gap of 2 microns (not to scale)

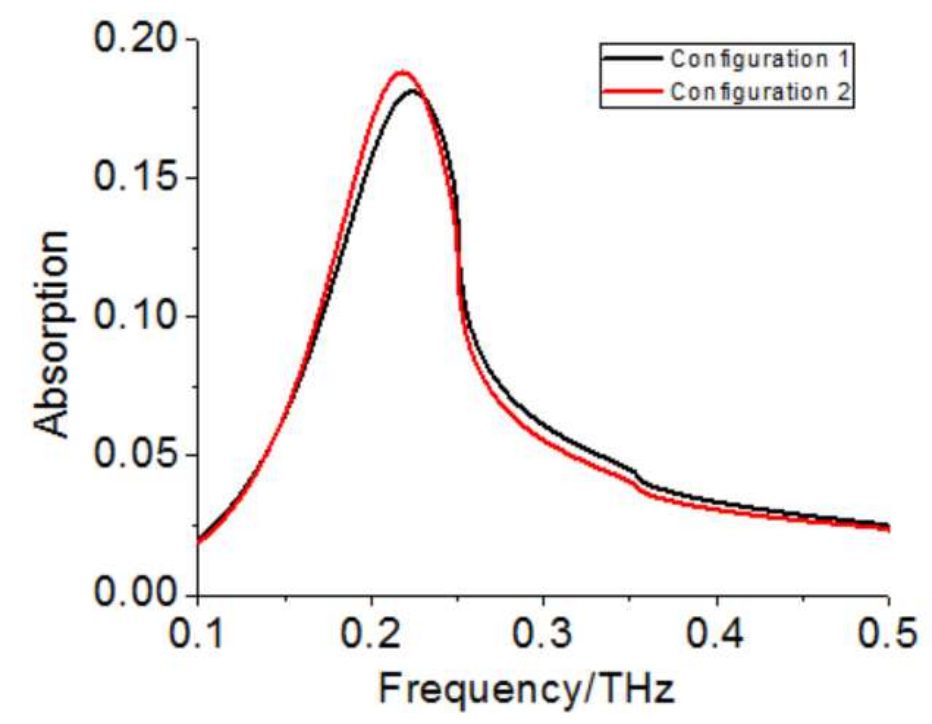

Figure 5.23. Response comparison between two resistor configurations 
For the second configuration, the contact area of the metal contact to the antenna/load is $0.8 * 0.8$ square micron. From Figure 5.23, response difference between these two configurations is not very considerable. Therefore, if certain tolerance is allowed, the first configuration, which requires less computation burden than the second one since it requires a smaller portion of finer mesh size, could be chosen as the model. Moreover, we have three different tests for variations of the distance $(6,8,10$ microns) between the two metal contacts. Similarly, the three curves are very close to each other (not shown).

Since the second configuration is more close to the actual case, here we plot the electric field and current density information in the highlighted region (yellow dash line) in the diagram of Configuration 2 . The mesh size of the plane is $0.2 * 0.2$ square micron.
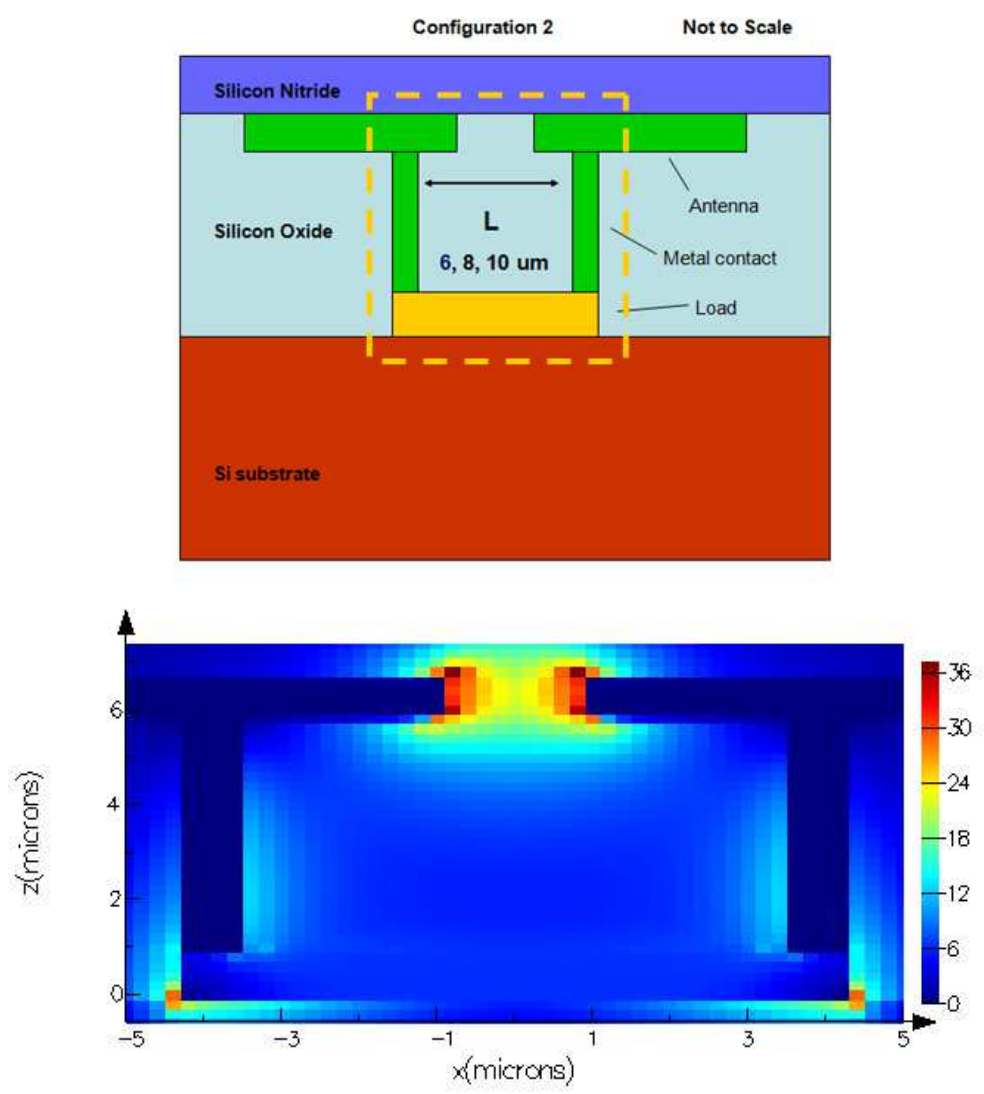

Figure 5.24. E-field distribution (@215THz) in a highlighted area (yellow dash line box) of Configuration 2. Electrical field unit: $\mathrm{V} / \mathrm{m}$ 


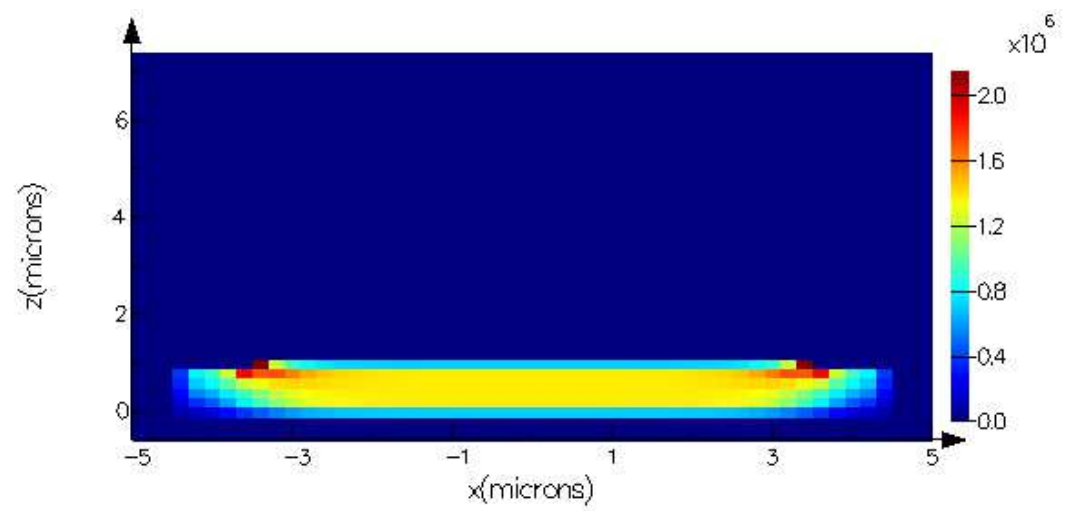

Figure 5.25. Current density distribution (@215THz) in the highlighted area in

Figure 5.24. Current density unit: $\mathrm{A} / \mathrm{m}^{2}$

For the E-field plot, the electrical intensity is high in the antenna gap. The field could not penetrate into the antenna and the metal contact. There is E field in the resistor. However, the current density concentrates itself in the resistor and peak values appear at two ends of the resistor.

\section{2. $188 \mathrm{GHz}$ transmission experiment design}

In order to verify our modeling results by experiments, we designed some antennas of study for experimental testing. Based on the source (a $94 \mathrm{GHz}$ Gunn diode and a frequency doubler) we have at the time of designing, the idea is that we could build multiple antennas and measure their transmission at $188 \mathrm{GHz}$. Five antennas are designed so that they have different transmission values (first decrease and then increase) at this specific frequency location. If the measurements agree with this trend for these five antennas, the simulation could be deemed reliable.

Three noticeable issues are: the difference of the five transmission values should be maximized so that they could be easily distinguished in measurement; also, considering the potential deviation of modeling results from the actual values, broadband antenna is desired; finally, two sets of antennas (one includes resistor and 
the other one does not include resistor, since we are not very sure about the validity of using this simple resistor model) are designed, totally ten antennas.

Bowtie antennas of 60 degrees are chosen as the design pattern, the central part of the antenna is a 5 um-by-5um square resistor $(50-\mathrm{ohm})$. The profile and GDS file of antenna of the two sets of design are shown in Figure 5.26. Thicknesses of aluminum and oxide layers are 1 and 2 microns respectively.

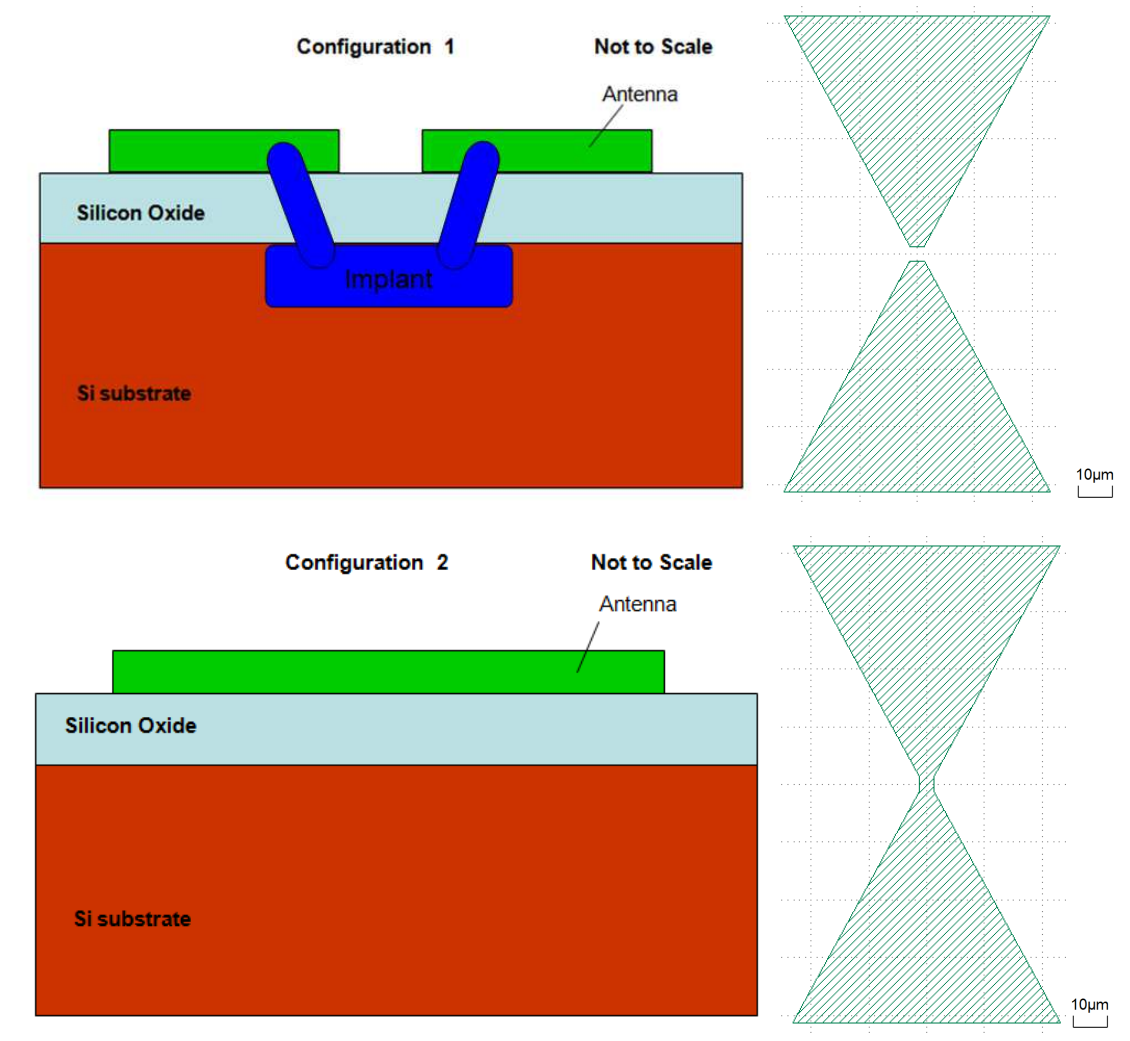

Figure 5.26. Two sets of antenna designs for $188 \mathrm{GHz}$ transmission experiment. In the first set, there is a gap between two arms of the antenna. Certain implant could be added to define an equivalent resistor. In the second set, there is no equivalent resistor and the two arms are directly connected with each other.

Within one set of antennas, the angle is fixed. The length is changed to adjust the frequency response of the antennas, therefore providing different transmission values at $188 \mathrm{GHz}$. The pixel pitch is also adjusted according to the length of the 
antenna. After many tests, five antennas of different length for each set are chosen to be manufactured. The corresponding transmission curves are shown here:
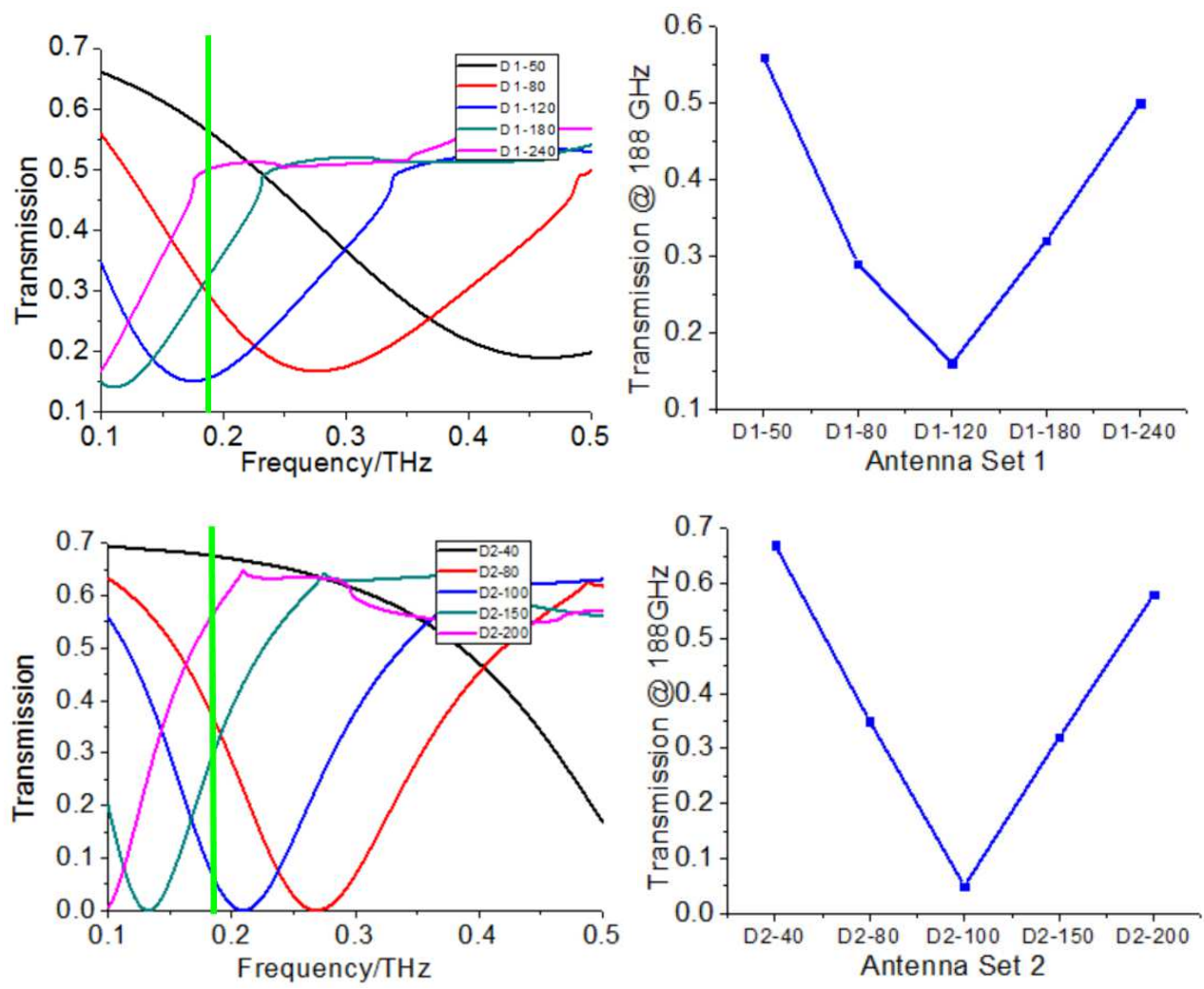

Figure 5.27. Transmission curves for two sets of antennas (left, the green line represents location of $188 \mathrm{GHz}$ ) and the transmission values at $188 \mathrm{GHz}$ (right)

The antenna parameters are listed in Table 5.3: 


\begin{tabular}{|c|c|c|c|c|}
\hline Design & Configuration & $\begin{array}{c}\text { Length of one } \\
\text { arm/micron }\end{array}$ & $\begin{array}{c}\text { Period/square } \\
\text { micron }\end{array}$ & Array \\
\hline Blank 1 & Bare silicon & - & - & - \\
\hline Blank 2 & $\begin{array}{c}\text { Silicon + } \\
\text { aluminum }\end{array}$ & - & $120 * 120$ & $125 * 125$ \\
\hline D1-50 & 1 & 50 & $180 * 180$ & $83 * 83$ \\
\hline D1-80 & 1 & 80 & $260 * 260$ & $57 * 57$ \\
\hline D1-120 & 1 & 120 & $380 * 380$ & $39 * 39$ \\
\hline D1-180 & 1 & 180 & $500 * 500$ & $30 * 30$ \\
\hline D1-240 & 1 & 240 & $100 * 100$ & $150 * 150$ \\
\hline D2-40 & 2 & 40 & $180 * 180$ & $83 * 83$ \\
\hline D2-80 & 2 & 80 & $220 * 220$ & $68 * 68$ \\
\hline D2-100 & 2 & 100 & $320 * 320$ & $46 * 46$ \\
\hline D2-150 & 2 & 150 & $35 * 35$ \\
\hline D2-200 & 2 & 200 & -420 & \\
\hline
\end{tabular}

Table 5.3. Antenna parameters for $188 \mathrm{GHz}$ transmission experiment. Period indicates the size of one pixel pitch. Array represents the number of antennas in $\mathrm{x}$ and y directions (the array size of each type of antenna is about $1.5^{*} 1.5$ square centimeters).

This experiment is still in progress and the experimental data should be compared to the right two plots of Figure 5.27. 


\subsection{Angular response of antenna}

One important issue we are interested in is that how the antenna response would be as the incident angle of incoming radiation changes. Previous modeling assumed all the incident radiation is normal to the plane where the antenna resides in. We could compare the angular response of the antenna to the already known antenna radiation pattern due to the reciprocity theorem (antenna works equally well as transmitters or receivers) [57].

To ensure the simulation is valid, we first need to verify two simple cases: angular transmission/reflection of air/silicon interface; angular absorption response of a dipole antenna of which the radiation pattern is known. After these two, the combination of them, antenna sitting on silicon substrate, could be implemented.

The transmission and reflection of air/silicon surface for different incident angles could be calculated from Fresnel equations [58] and simulated from FDTD Solutions (for component parallel to the incident plane):

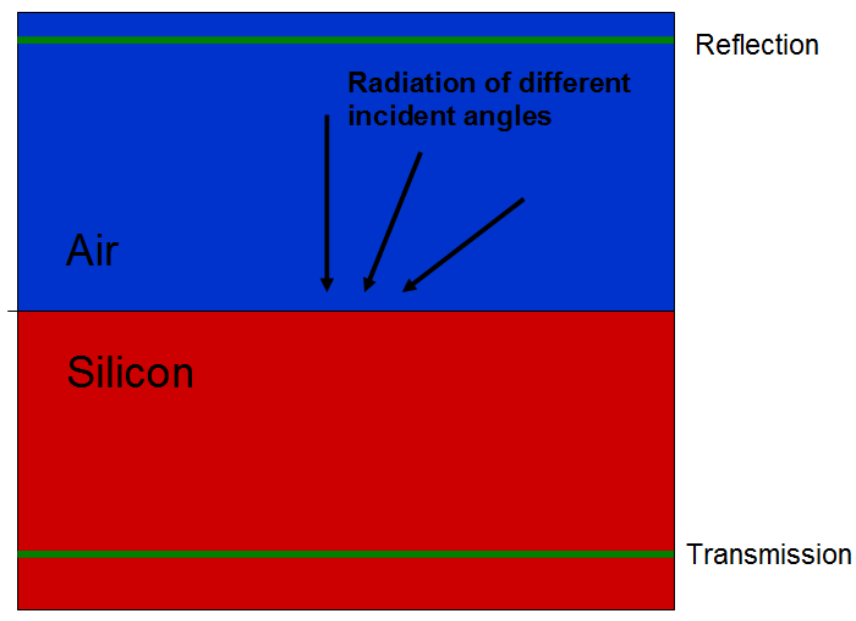

Figure 5.28. Transmission and reflection of air/silicon interface for radiation of different incident angles 

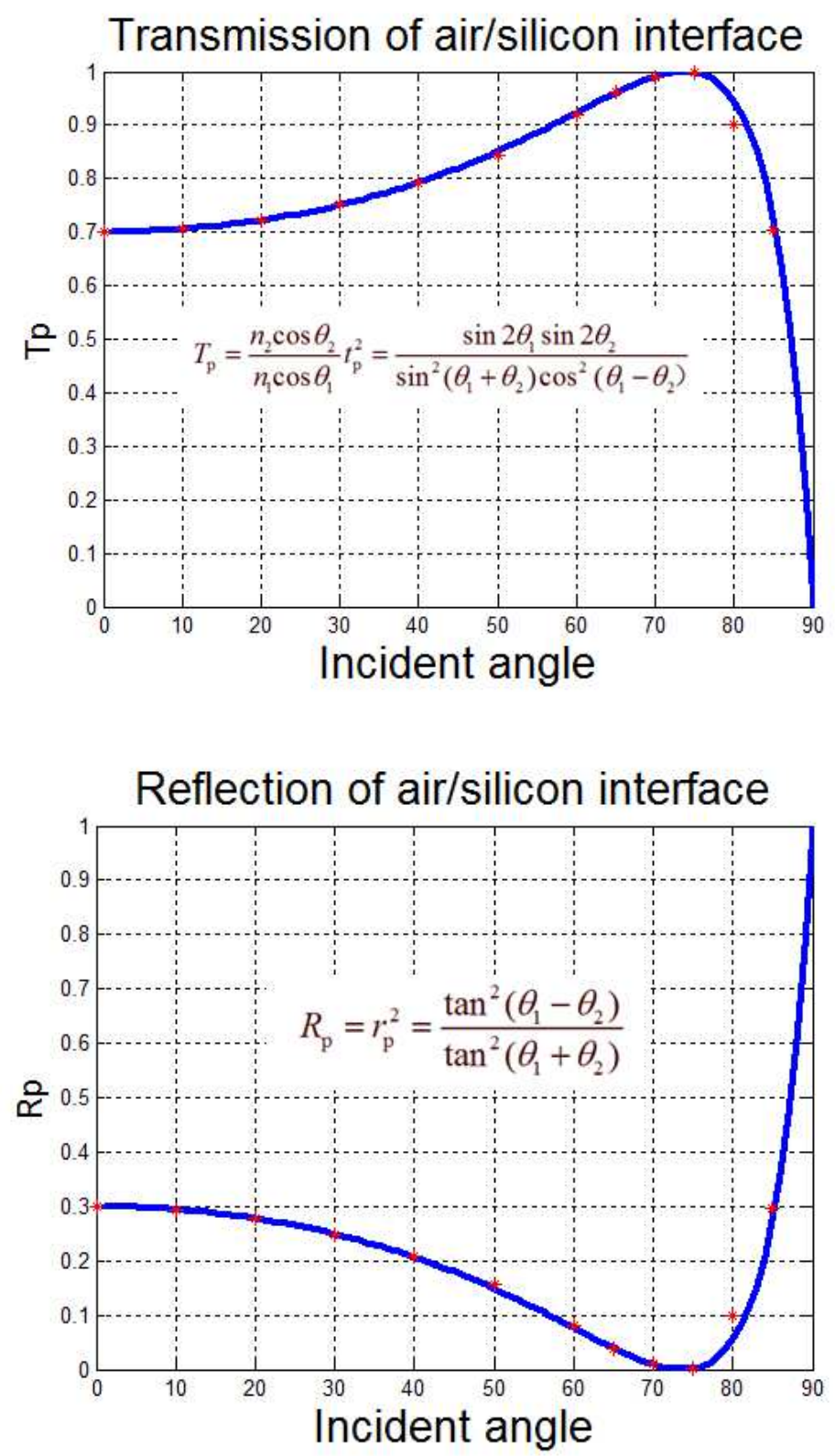

Figure 5.29. Comparison between theoretic and simulation results for transmission and reflection of air/silicon interface as a function of incident angle. Blue curves are of theory (Fresnel equations in [58]) and red stars are of simulation results. $T_{p}$ and $R_{p}$ are the transmission and reflection rates ( $t_{p}$ and $r_{p}$ give the amplitude). $\theta$ is the incidence/refraction angle and $\mathrm{n}$ represents the refractive index ( 1 is air and 2 is silicon). Source frequency is $0.18 \mathrm{THz}$. For data points of angle up to 60 degrees, the number of PML layers is 48; for all other cases with angle larger than 60 degrees, the number of PML layers is set to be 96 . 
The comparison between Fresnel equations and simulation results show that they agree well for smaller angles while for larger angles special attentions need to be paid. One characteristic for the Perfect Matched Layer (PML, boundary condition used in Lumerical to avoid energy reflection) in the $\mathrm{z}$ direction is that it does not perform well for large angles [59]. The Lumerical website [60] indicates that when the incident angle is larger than 65 degrees, the number of PML layer should be increased and careful tests need to be done to ensure convergence. During out tests, when there are 48 PML layers, the transmission for some angles even exceeds 1 , which is not physical. In our final results, when the incident angles are larger than 60 degrees, 96 layers are applied. This makes all the data points to be physical, however, for 80 degrees, the deviation is still obvious. This effect is more obvious for the next dipole antenna case.

The second set of tests is for dipole antenna (in free space) absorption at different incident angles (Figure 5.30). There is no silicon substrate included. The theoretical formula used is from [62]

$$
\bar{S}_{a v}=\hat{a}_{r} \frac{\beta^{2} \eta}{32 \pi^{2}}|I|^{2}(\Delta L)^{2} \frac{\sin ^{2} \theta}{r^{2}}\left[\mathrm{~W} / \mathrm{m}^{2}\right](\beta r \gg 1)
$$

The time-average radiated power $\overline{S_{a v}}$ is related to the radius $\mathrm{r}$, antenna length $\Delta \mathrm{L}$, incident angle $\theta$, wave vector $\beta$, current $I$ (ar is the directional unit vector) and $\eta=$ $\beta /(\varpi \varepsilon)$ ( $\omega$ is the angular frequency of the radiation, $\varepsilon$ is the permittivity of the material). After fixing all other parameters, the radiation is proportional to squared sinusoid of the incident angle $\theta$. The comparison between theory and simulation is shown in Figure 5.31. 

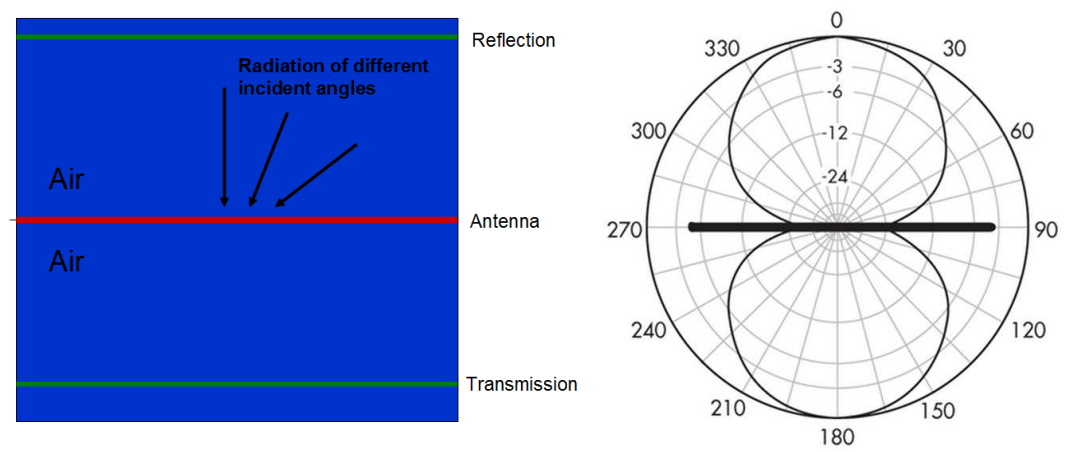

Figure 5.30. Left: Angular absorption response of a dipole antenna. The antenna includes a resistor to absorb energy and the absorption is equal to one minus reflection and transmission $(A=1-R-T)$. Right: An example of $E$ field radiation pattern of a half-wave dipole antenna [59]. The antenna elements are located along the $270^{\circ}$ and $90^{\circ}$ Line.

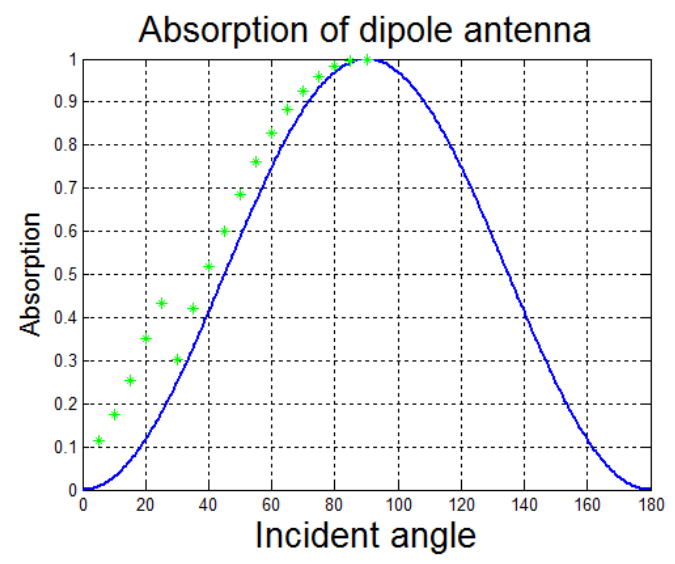

Figure 5.31. Comparison between theoretic and simulation results for absorption of dipole antenna as a function of incident angle. Blue curve is of theory and green stars are of simulation results. Source frequency is $0.18 \mathrm{THz}$. Different from Figure 5.29 and 5.30, here 90-degree corresponds to the normal incidence and small angles represent larger incident angles. For data points of angle up to 25 degrees, the used number of PML layers is 48; for all other cases with angle larger than 25 degrees, the number is set to be 24 .

It should be indicated that the dipole antenna utilized here is 245 microns long, 24 microns wide and thick, which makes the aspect ratio to be about 10 . We have tried 
another case where width and thickness were both 5 microns and the length was 245 microns. The comparison shows that simulation results for the wider dipole (24 microns) are more close to the theoretical curve. The aspect ratio may be a factor affecting how close the simulations and theories would be.

To get more accurate simulations, three improvements were implemented: firstly, for larger incident angles, 48 PML layers were used; secondly, finer mesh size of $1 * 1$ square micron was used; thirdly, the relative location of the reflection monitor and the source was adjusted, making the monitor between the source and the dipole subject. The fact that the algorithm does not perform well at larger incident angles is clearer for our dipole antenna angular absorption modeling than the previous transmission/reflection simulation at air/silicon interface. The number of PML layers may be increased even larger to reduce the error.

Since these two sets of tests got good results for incident angles less than 60 degrees (theoretical and simulation data roughly agree except for some data points at large incident angles), we could proceed to have a look at the angular response of our designed bowtie antenna. Some initial simulation results are not intuitive so that additional effort needs to be made in the future.

\subsection{Conclusion and future work}

The focus of this thesis is to simulate $\mathrm{THz}$ antennas, using the commercial FDTD software, Lumerical FDTD solutions. For absorption simulation, a mathematically equivalent resistor is included in the model to transfer $\mathrm{THz}$ light into electrical power. Using PEC as the aluminum antenna material model is fast and the results are close to the cases using aluminum Drude model. Through many tests, we get knowledge of how various parameters in the design would affect the performance of the antenna. The desired antenna design in order to couple incident radiation should 
be compact. Most of our simulation results are physical or could be directly compared to some existing references. There is a linear relationship between the bowtie antenna length and resonant wavelength, which could help design certain types of antenna for specific interested spectral bands. Some issues during the manufacture, like the slots to qualify for the design rule for discontinuity, were addressed. Some other information, such as E-field distribution, current density, were also provided for enhancing the understanding. The absorption antenna for $215 \mathrm{GHz}$ and transmission experiment at $188 \mathrm{GHz}$ were designed and could serve to verify the validity of the simulation.

In the future, research on some specific deficiencies should be taken into account. The index of refraction data for materials within terahertz range are not available and need to be measured. After the $188 \mathrm{GHz}$ transmission experiment and $215 \mathrm{GHz}$ antenna designs help further verifying the validity of our simulation model, this model needs to get more complicated to be more close to the actual structure. 


\section{References}

[1] https://nelson.mit.edu/blog/terahertz-polaritonics

[2]

http://www.toptica.com/products/terahertz_generation/terahertz_technologysources_and_thz_gen eration_methods/properties_of_terahertz_radiation.html

[3] Gharavi, Sam; Heydari, Babak (2011-09-25). Ultra High-Speed CMOS Circuits : Beyond 100 GHz (1st ed.). New York: Springer Science+Business Media. pp. 1-5 (Introduction) and 100.

[4] Sirtori, Carlo (2002). "Applied physics: Bridge for the terahertz gap" . Nature 417 (6885): $132-3$

[5] Borak, A. (2005). "Applied physics: Toward Bridging the Terahertz Gap with Silicon-Based Lasers" Science 308 (5722): 638-9.

[6] Karpowicz, Nicholas; Dai, Jianming; Lu, Xiaofei; Chen, Yunqing; Yamaguchi, Masashi; Zhao, Hongwei; Zhang, X.-C.; Zhang, Liangliang; Zhang, Cunlin; Price-Gallagher, Matthew; Fletcher, Clark; Mamer, Orval; Lesimple, Alain; Johnson, Keith (2008). "Coherent heterodyne time-domain spectrometry covering the entire "terahertz gap"". Applied Physics Letters (Abstract) 92: 011131

[7] Kleiner, R. (2007). "Filling the Terahertz Gap". Science (Abstract) 318 (5854): 1254-5

[8] Arnone, Don, Craig Ciesla, and Michael Pepper. "Terahertz imaging comes into view." Physics World 4 (2000): 35-40.

[9] http://www.dlr.de/pf/desktopdefault.aspx/tabid-833/1333_read-2400/

[10] JLab generates high-power terahertz light http://cerncourier.com/cws/article/cern/28777

[11] Standoff identification of concealed explosives under real-world conditions

[12] Zhao, Jiayu, et al. "Terahertz imaging with sub-wavelength resolution by femtosecond laser filament in air." Scientific reports 4 (2014).

[13] http://www.digitalbarriers.com/products/thruvision/thruvision-ts5/

[14] Sengupta, Kaushik, and Ali Hajimiri. "A 0.28 THz power-generation and beam-steering array in CMOS based on distributed active radiators." Solid-State Circuits, IEEE Journal of 47.12 (2012): 3013-3031.

[15] http://www.z-thz.com/index.php?option=com_content\&view=article\&id=51\&Itemid=59

[16] http://www.teraview.com/applications/medical/oncology.html 
[17] Nagatsuma, Tadao, et al. "Terahertz wireless communications based on photonics technologies." Optics express 21.20 (2013): 23736-23747.

[18] Akyildiz, Ian F., Josep Miquel Jornet, and Chong Han. "Terahertz band: Next frontier for wireless communications." Physical Communication 12 (2014): 16-32.

[19] Jördens, Christian, Frank Rutz, and Martin Koch. "Quality assurance of chocolate products with terahertz imaging." European Conference on NDT. 2006.

[20] Rogalski, A., and F. Sizov. "Terahertz detectors and focal plane arrays." Opto-electronics review 19.3 (2011): 346-404.

[21] A.D. Turner, J.J. Bock, J.W. Beeman, J. Glenn, P.C. Hargrave, V.V. Hristov, H.T. Nguyen,

F. Rahman, S.Sethuraman, and A.L. Woodcraft, "Silicon nitride micro-mesh bolometer array for submillimeter astrophysics", Appl.Optics 40, 4921-4932 (2001).

[22] "Detectors needs for long wavelength astrophysics", A Report by the Infrared,

Submillimeter, and Millimeter Detector Working Group, June 2002;

http://safir.gsfc.nasa.gov/docs/ISMDWG_final.pdf

[23] J. Glenn, P.A.R. Ade, M. Amarie, J.J. Bock, S.F. Edgington, A. Goldin, S. Golwala, D.

Haig, A.E. Lange, G. Laurent, P.D. Maudkopf, M. Yun, and H. Nguyen, "Current status of

Bolocam: a large-format millimeter-wave bolometer camera”, Proc. SPIE 4855, 30-40 (2003).

[24] G.M. Voellmer, C.A. Allen, M.J. Amato, S.R. Babu, A.E.Bartels, D.J. Benford, R.J. Derro,

C.D. Dowell, D.A. Harper, M.D. Jhabvala, S.H. Moseley, T. Rennick, P.J. Shirron, W.W. Smith,

and J.G. Staguhn, "Design and fabrication of two-dimensional semiconducting bolometer arrays for HAWC and SHARC-II", Proc. SPIE 4855, 63-72 (2003).

[25] J.G. Staguhn, D.J. Benford, F. Pajot, T.J. Ames, J.A.Chervenak, E.N. Grossman, K.D. Irwin, B. Maffei, S.H.Moseley, T.G. Phillips, C.D. Reintsema, C. Rioux, R.A. Shafer, and G.M. Vollmer, "Astronomical demonstration of superconducting bolometer arrays", Proc. SPIE 4855,100-107 (2003).

[26] C.M. Bradford, B.J. Naylor, J. Zmuidzinas, J.J. Bock, J.Gromke, H. Nguyen, M. Dragovan, M. Yun, L. Earle, J.Glenn, H. Matsuhara, P.A.R. Ade, and L. Duband, "WaFIRS: A waveguide far-IR spectrometer: Enabling spectroscopy of high-z galaxies in the far-IR and submillimeter",Proc. SPIE 4850, 1137-1148 (2003)

[27] Webster, John G (1999). The measurement, instrumentation, and sensors handbook. pp. 32113.

[28] http://en.wikipedia.org/wiki/Julius_Edgar_Lilienfeld

[29] http://www.elprocus.com/mosfet-as-a-switch-circuit-diagram-free-circuits/ 
[30] Dyakonov, Michael, and Michael Shur. "Shallow water analogy for a ballistic field effect transistor: New mechanism of plasma wave generation by dc current." Physical review letters 71.15 (1993): 2465.

[31] Evaluation of MOSFETs for Terahertz Detector Arrays. Gregory Fertig. Master Thesis, Rochester Institute of Technology, 2014

[32] Knap, W., et al. "Nonresonant detection of terahertz radiation in field effect transistors." Journal of Applied Physics 91.11 (2002): 9346-9353.

[33] El Fatimy, Abdelouahad, et al. "Resonant and voltage-tunable terahertz detection in InGaAs InP nanometer transistors." Applied physics letters 89.13 (2006): 131926.

[34] Meziani, Yahya Moubarak, et al. "Non resonant response to terahertz radiation by submicron CMOS transistors." IEICE transactions on electronics 89.7 (2006): 993-998.

[35] Dyer, Gregory C., et al. "A narrowband plasmonic terahertz detector with a monolithic hot electron bolometer." SPIE OPTO: Integrated Optoelectronic Devices. International Society for Optics and Photonics, 2009.

[36] Knap, Wojciech, et al. "Field effect transistors for terahertz detection: physics and first imaging applications." Journal of Infrared, Millimeter, and Terahertz Waves 30.12 (2009): 13191337.

[37] Knap, W., et al. "Plasma excitations in field effect transistors for terahertz detection and emission." Comptes Rendus Physique 11.7 (2010): 433-443.

[38] Knap, W., et al. "Plasma wave detection of sub-terahertz and terahertz radiation by silicon field-effect transistors." Applied Physics Letters 85.4 (2004): 675-677.

[39 Teppe, F., et al. "Room temperature tunable detection of subterahertz radiation by plasma waves in nanometer InGaAs transistors." Applied physics letters89.22 (2006): 222109-222109.

[40] www.lumerical.com

[41] https://www.lumerical.com/tcad-products/fdtd/

[42] https://rc.fas.harvard.edu/resources/odyssey-architecture/

[43] http://www.circ.rochester.edu/news.html\#

[44] Grischkowsky, D., et al. "Far-infrared time-domain spectroscopy with terahertz beams of dielectrics and semiconductors." JOSA B 7.10 (1990): 2006-2015.

[45] Cataldo, Giuseppe, et al. "Infrared dielectric properties of low-stress silicon nitride." Optics letters 37.20 (2012): 4200-4202.

[46] Wen-Feng, Sun, Wang Xin-Ke, and Zhang Yan. "Measurement of refractive index for high reflectance materials with terahertz time domain reflection spectroscopy." Chinese Physics Letters 26.11 (2009): 114210. 
[47] Ordal, M. A., et al. "Optical properties of the metals al, co, cu, au, fe, pb, ni, pd, pt, ag, ti, and $\mathrm{w}$ in the infrared and far infrared." Applied Optics 22.7 (1983): 1099-1119.

[48] Lumerical training slides for Material properties

[49] Edward D. Palik. Handbook of Optical Constants of Solids, Volume 2. Academic Press, 1991

[50] Singh, Ranjan, et al. "Spiral-type terahertz antennas and the manifestation of the Mushiake principle." Optics express 17.12 (2009): 9971-9980.

[51] Fischer, Holger, and Olivier JF Martin. "Engineering the optical response of plasmonic nanoantennas." Optics Express 16.12 (2008): 9144-9154.

[52] http://www.antenna-theory.com/antennas/halfwave.php

[53] Schuster, Franz, et al. "THz imaging with low-cost 130 nm CMOS transistors."Security+ Defence. International Society for Optics and Photonics, 2010.

[54] Schuster, Franz, et al. "Broadband terahertz imaging with highly sensitive silicon CMOS detectors." Optics Express 19.8 (2011): 7827-7832.

[55] Fumeaux, C., et al. "Nanometer thin-film Ni-NiO-Ni diodes for detection and mixing of 30 THz radiation." Infrared physics \& technology 39.3 (1998): 123-183.

[56] Yu, Nanfang, et al. "Bowtie plasmonic quantum cascade laser antenna." Optics Express 15.20 (2007): 13272-13281.

[57] http://en.wikipedia.org/wiki/Reciprocity_(electromagnetism)

[58] Hecht, Eugene (1987). Optics (2nd ed). Addison Wesley. ISBN 0-201-11609-X.

[59] http://electronicdesign.com/wireless/what-s-difference-between-dipole-and-ground-planeantenna

[60] Notes on Perfectly Matched Layers (PMLs) Steven G. Johnson Created August 2007; updated March 10, 2010

[61] http://docs.lumerical.com/en/index.html?ref_sim_obj_broadband_injection_angles.html [62] Lecture notes \#33. K.W.Whites, EE 382 Applied Electromagnetics 